\title{
UCRL-10904
}

MASTER

University of California

Ernest 0 . Lawrence Radiation Laboratory

\section{MUTUAL CHARGE NEUTRALIZATION OF GASEOUS IONS}

\author{
Berkeley, California
}




\section{DISCLAIMER}

This report was prepared as an account of work sponsored by an agency of the United States Government. Neither the United States Government nor any agency Thereof, nor any of their employees, makes any warranty, express or implied, or assumes any legal liability or responsibility for the accuracy, completeness, or usefulness of any information, apparatus, product, or process disclosed, or represents that its use would not infringe privately owned rights. Reference herein to any specific commercial product, process, or service by trade name, trademark, manufacturer, or otherwise does not necessarily constitute or imply its endorsement, recommendation, or favoring by the United States Government or any agency thereof. The views and opinions of authors expressed herein do not necessarily state or reflect those of the United States Government or any agency thereof. 


\section{DISCLAIMER}

Portions of this document may be illegible in electronic image products. Images are produced from the best available original document. 
UCRL-10904

UC - 4 Chemistry.

TID-4500 (19th Ed.)

\section{UNIVERSITY OF CALIFORNIA}

Lawrence Radiation Laboratory

Berkeley, California

Contract No. W-7405-eng-48

MUTUAL CHARGE NEUTRALIZATION OF GASEOUS IONS

James Carl Person

(Thesis)

July 11, 1963 
Printed in USA. Price $\$ 2.50$. Available from the office of Technical Services

U. S. Department of Cummerce Washingt on 25 , D.C. 
MUTUAL CHARGE NEUTRALIZATION OF GASEOUS IONS

\section{Contents}

Abstract

I. Introduction

II. Nature of the Ionized Gas
A. Photoionization
B. Disappearance of the Photoelectron . . . . . 4
C. Fate of the Ions Initially Produced . . . . . 6

III. Experimental Apparatus and Procedure
A. Procedure
B. Light Source
C. Lamp Intensity Monitor
D. Collecting Voltage
E. Reaction Cel
F. Gas Purity
G. Experimental Difficulties

IV. Estimation of Ion Mobilities

A. Description of the Method of Computation . . . 22

B. Results of Computer Calculations . . : . . 26

C. Relation Between the Mobility and the Nature of the Ions

D. Discussion of the Results of the Mobility Determination

V. Results of the Experimental Determination of the Recombination Coefficient, a
A. Second-Order Process
B. Initial Recombination
C. Effects of Pressure and of Different Third-Body
Gasés on a . . . . . . . . . . . . . 49
D. Determination of the Low Pressure Limit of a . $\quad 51$
E. Termolecular Charge Neutralization . . . . 58

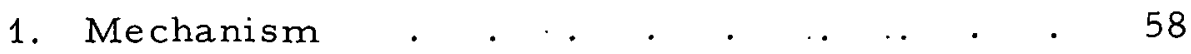
2. Experimental Determination of $k_{1} \cdot{ }_{1} \cdot 60$ 
3. Estimation of $\mathrm{k}_{2}$. . . . . . . . . 64

4. Determination of the Relative Third-Body Efficiencies and the Estimation of $\mathrm{k}_{3}$. . . 65

VI. Comparison of Results with Existing Theories

A. Comparison of: Results with the Theory of Fueno, Eyring, and Ree . . . . . . . . . 67

B. Thomson Theory . . . . . . . . . 75

C. Natanson Theory . . . . . . . . . . 76

D. Modifications Required When the Ion Mass and the Neutral Mass Are Unequal . . . . . . . . 80

VII. Detailed Calculation of the Collisional Deactivation Merhansim

A. Basic Assumptions . . . . . . . . . . 89

B. Requirements for Deactivation . . . . . . 90

C. Calculation of the Rate of Obtaining a Deflection $x_{1 \mathrm{M}} 96$

D. Calculation of the Rate of Collisional Deactivation . 99

E. Calculation of the Recombination Coefficient, $a_{T} \cdot 102$

F. Results of the Detailed Calculation . . . . . 106

Acknowledgments . . . . . . . . . . . . . . . 116

Symbols : . . . . . . . . . . . . . . 117

Appendixes

A. FORTRAN-II Program for Calculation of. Induced Curleint vs Tirise Curves . . . . . . . 118

B. Rate of Obtaining Deflecliun Angles in $\Delta x_{1 M}$ Regions 122

C. FORTRAN-II Program for the Detailed Calculation of a . . . . . . . . . . . . . . 123

References . . . . . . . . . . . . . . . 138 
MUTUAL CHARGE NEUTRÁLIZATION OF GASEOUS IONS

James Carl Person

Lawrence Radiation Laboratory .

University of California

Berkeley, California

July 11,1963

\begin{abstract}
We consider the problem of the bimolecular rate constant, $a$, for the mutual charge neutralization reaction (ion-ion recombination) for ions formed by the vacuum ultraviolet photolysis of nitric oxide. We measure the pressure dependence of a over a pressure range of 10 to 600 torr for mixtures of a few hundred microns of NO with He, Ar, $\mathrm{Kr}, \mathrm{Xe}, \mathrm{H}_{2}, \mathrm{D}_{2}$, and $\mathrm{N}_{2}$.

From the low-pressure limit of $a$, we determine that the rate constant for charge neutralization in the absence of a third body is $\mathrm{k}_{0}=2.1 \pm 0.4 \times 10^{-7} \mathrm{~cm}^{3} / \mathrm{sec}$. We estimate the high-pressure limit of $a$ to be $2.0 \pm 0.5 \times 10^{-6} \mathrm{~cm}^{3} / \mathrm{sec}$. We measure the third-body efficiencies for promoting the charge-neutralization reaction; the results, relative to $\mathrm{He}$ as the third-body gas, are $\mathrm{H}_{2}:=1.4 \pm 0.4, \mathrm{D}_{2}=1.5 \pm 0.4$, $\mathrm{Ar}=3.6 \pm 0.8, \mathrm{Kr}=4.3 \pm 1.0, \mathrm{~N}_{2}=5.2 \pm 1.1$, and $\mathrm{Xe}=6.8 \pm 1.5$.

We estimate the average ionic mobility in the gas mixtures, and the mobilities indicate that at least some of the ions must be present as ion clusters. Also, we show that the addition of $\mathrm{NO}_{2}$ or $\mathrm{H}_{2} \mathrm{O}$ further lowers the mobility.

Finally, we make a detailed calculation of the three-body chargeneutralization process, using a computer. This calculation considers that the rate of charge neutralization is the rate at which ion pairs are deactivated by collision with the neutral gas molecules to form ion pairs which cannot separate to large distances. The potential between the ions and the neutrals is assumed to be an ion-induced dipole potential with a hard-sphere core. The calculation involves an average over the
\end{abstract}


various angles in the collisions. The predicted values of a depend on a parameter of the calculation, but over a wide range of this parameter the predicted relative third-body efficiencies are in reasonable agreement with the experimental values. 


\section{INTRODUCTION}

In radiation chemistry and in the study of the upper atmosphere, we consider situations in which considerable concentrations of gaseous ions may be present. Because these ions undergo a wide variety of ion-neutral reactions, which can be quite different from reactions between neutral species, ${ }^{1}$ it is important to know how large the ion concentrations are under various experimental conditions. One thing that determines the ion concentration is the rate of the mutual charge-neutralization reaction--the so-called ion-ion recombination reaction--in which a positive ion and a negative ion react to give uncharged products.

Early studies of the rates of recombination were done using impure gases under conditions in which the ion concentrations were not uniform, so that the results are questionable. ${ }^{2,3}$ In reviewing the results of ion-recombination studies done before 1955 in the pressure region of a few torr to 1 to 2 atm, Loeb ${ }^{3}$ considered only two experiments worthy of consideration, Gardner's study of the recombination of ions formed by passing $x$ rays through $\mathrm{O}_{2}$, published in 1938, 4 and Sayers' work on the recombination of ions formed by $x$ rays in air, also published in 1938. ${ }^{5}$ Since 1955, Yeung has presented reports on the value of the specific rate of recombination of ions in $\mathrm{I}_{2}$ at low pres sures, ${ }^{2,6}$ the rate for ions in $\mathrm{Br}_{2},{ }^{2,6}$ as well as a brief report on the rate for cesium ions with iodine ions. ${ }^{7}$ However, few new data have been presented.

When the total gas pressure is below 1 or 2 atm, the theory generally used to explain the increase in the specific rate of ion recombination with increasing pressure is that proposed by Thomson ${ }^{8}$ or some modification of it, such as Natanson has given. ${ }^{9}$ In the Thomson theory it is assumed that when the positive and the negative ions collide, following open orbits because of their initial kinetic energy of relative motion, the ions separate again to large distances with only a small chance of charge neutralization. However, if the ions collide with a neutral gas molecule while they are relatively close together, the collision may cause the ions to lose enough kinetic energy of relative 
motion to make triem unable to separate to large distances. The ions are then in a closed orbit, where they eventually neutralize each other.

Recently, Fueno, Eyring, and Ree have proposed an alternative mechanism for ion recombination in the presence of a third body. ${ }^{10}$ In this mechanism the neutral molecule first forms an ion complex with one of the ions, then this complex reacts with the other ion, and the neutral removes the excess energy. This theory is analogous to the complex-formation mechanism proposed for atom recombination, ${ }^{11}$ whilc the Thomson theory ic analogoue to the collisional deactivation mechanism tor atom recombination.

We decided to study the effects of pressure on the rate constant for ion recombination when different gases were used as the third-body gases. In this way we were able to obtain values of the relative thirdbody efficiencies for enhancing ion recombination

The ions were formed by photolysis of nitric oxide, and we were able to produce larger initial ion concentrations than were Sayers and Gardner, so that we could follow the recombination over a larger fractional change in the ion concentration. Also, because of our greater ion concentrations, we were able to use lower pressures, since diffusional losses were less of a problem--we could therefore study the low-pressure limit of the rate constant for ion recombination. as well: as the approach to the high-pressure limit.

Section IV gives the results of rough measurements we made of the ionic mobility in our attempt to get direct evidence about the nature of the ions involved. Finally, Sec. VII describes the detailed calculation we made using the collisional deactivation mechanism to predict the relative third-body efficiencies. 


\section{NATURE OF THE IONIZED GAS}

The system studied consisted of NO at a pressure of around $300 \mu$ in a mixture with an inert gas to give a total pressure of 3 to 600 tor $x$. Vacuum ultraviolet photolysis produced an initial ion concentration of ( 3 to 10$) \times 10^{8}$ ions $/ \mathrm{cm}^{3}$ - -in more common chemical terms, around $10^{-12}$ mole of ions per liter or a partial pressure a round $10^{-8}$ tor $r$. The average distance between ions was $r_{0} \approx n^{-1 / 3} \approx 1$ to $1.5 \times 10^{-3}$ $\mathrm{cm}$ (where $\mathrm{n}$ is the ion concentration). The usual picture of an "ideal" gas is based upon the assumption of independerit rrulecules, and since the Coulomb force between the ions is such a long-range force, the ions stop behaving independently at much lower concentrations than do neutral species. However, since the Coulomb potential energy at the average distance of separation between ions was only about $10^{-16}$ erg (much smaller than the average the rmal energy of $6 \times 10^{-14} \mathrm{erg}$ ), we see that we may still regard the ions as behaving independently at the ion concentrations used in the se experiments.

\section{A. Photoionization}

Absorption of light whose wavelength was shorter than $1340 \AA$ produced charged particles by

$$
\therefore \mathrm{NO}+\mathrm{hv} \rightarrow \mathrm{NO}^{+}+\mathrm{e} .
$$

The rate of production of ions, $q$, was $5 \times 10^{10}$ to $6 \times 10^{11}$ ions $/\left(\mathrm{cm}^{3} \mathrm{sec}\right)$. In most of the experiments, $1236-\AA$ light was used, so that the $\mathrm{NO}^{+}$ions were produced in the $v=0, v=1$, and $v=2$ vibrational levels, where the photoelectrons had kinetic energies of $0.77,0.47$, or $1.17 \mathrm{eV}$. Watanabe el al., have shown experimentally that the light absorption causes the populations of the $\mathrm{v}=0, \mathrm{v}=1$, and $\mathrm{v}=2$ vibrational levels of the $\mathrm{NO}^{+}$to be in the proportions of $0.79: 1.00: 1.0: 5,{ }^{12}$ and theoretical calculations of the transition probabilities agree qualitatively. with this result. 13,14 
B. Disappearance of the Photoelectron

These experiments were designed to study ion-ion recombination, so we want to assure ourselves that most of the photoelectrons attach to form negative ions before they have a chance to undergo ion-electron recombination. The photoelectrons produced in $E_{4} .(D-1)$ may have formed negative ions with any electronegative gas present (such as the $\mathrm{NO}$, or with the small amounts of $\mathrm{NO}_{2}, \mathrm{O}_{2}$, or $\mathrm{N}_{2} \mathrm{O}$, etc. that may also have been present). Two mechanisms were likely:

A three-body attachment reaction,

$$
\begin{gathered}
e+B+M^{\stackrel{k_{3}}{\longrightarrow}} B^{-}+M, \\
\text { e.g., e+NO+M } \rightarrow N O+M ;
\end{gathered}
$$

or a dissociative attachment reaction,

$$
\begin{gathered}
e+C \stackrel{k_{2}}{\longrightarrow} B^{-}+D \\
\text { e.g. } e+N_{2} \mathrm{O} \rightarrow \mathrm{O}^{-}+\mathrm{N}_{2}
\end{gathered}
$$

Few three-body attachment rates have been measured. Gunton and Inn give a rough value of $4 \times 10^{-31} \mathrm{~cm}^{6} / \mathrm{sec}$ for $\mathrm{k}_{3}$ in pure NO, ${ }^{15}$ and the third-order attachment for electrons of thermal energy to $0.6 \mathrm{eV}$ in pure $\mathrm{O}_{2}$ is reported to be $\mathrm{k}_{3}=(2$ to 4$) \times 10^{-30} \mathrm{~cm}^{6} / \mathrm{sec}$, with the $\mathrm{k}_{3}$ alvul $1 / 50$ as large if $\mathrm{N}_{2}$ is the third body instead of $\mathrm{O}_{2} \cdot 1 \mathrm{~h}$ An example of a two =bndy a.t.a.chment rate constant is $k_{2}=10^{-12}$ to $10^{-11} \mathrm{~cm}^{3} / \mathrm{sec}$ for $\mathrm{O}_{2}$ with electrons of 1.5 to $2.0 \mathrm{eV}$ energy. ${ }^{16}$

The electron might also have undergone an ion-electron recombination reaction with the NO!

$$
\mathrm{NO}^{+}+\mathrm{e} \stackrel{\mathrm{a}_{e}}{\longrightarrow} \text { neutral products. }
$$

Doering and Mahan reported $a_{e}$ as $(0.4$ to 2.0$) \times 10^{-6} \mathrm{~cm}^{3} / \mathrm{sec}_{1}$, and Gunton and Inn determined $a a$ to be $1.3 \times 10^{-6} \mathrm{~cm}^{3} / \mathrm{sec}^{15}$ Electron loss by diffusion to the walls may be neglected in comparison with the loss by ion-electron recombination at the pressure used. The firstorder decay constant for loss by diffusion can be taken as $D / \Lambda^{2}$, where 
$D$ is the diffusion coefficient and $\Lambda$ is the characteristic diffusion length. The value of the ambipolar diffusion coefficient can be estimated as ${ }^{18}$

$$
\mathrm{D} \approx 2(0.0235) \mathrm{k} \mathrm{cm}^{2} / \mathrm{sec} \text {, }
$$

where $\mathrm{k}$ is the positive-ion mobility in $\mathrm{cm}^{2} /$ (volt sec). The'use of a typical value of $k \approx 2.5(760 / \mathrm{P}) \mathrm{cm}^{2} /($ volt sec) gives $\mathrm{D} \approx 90 / \mathrm{P} \mathrm{cm} / \mathrm{sec}$, where $P$ is the pressure in torr. Estimating $\Lambda$ to be 0.4 to $2 \mathrm{~cm}$ gives $D / \Lambda^{2} \approx 22 / \mathrm{P}$ to $560 / \mathrm{P} \sec ^{-1}$ as the decay constant for electron loss by diffusion. In contrast, the first-order decay constant for electronion.. recombination is $\mathrm{a}_{\mathrm{e}}\left[\mathrm{n}_{+}\right]$, which can be estimated by taking $a_{e} \approx 1 \times 10^{-6} \mathrm{~cm}^{3} / \mathrm{sec}$ and $\left[n_{+}\right] \approx 6 \times 10^{8}$ ion $/ \mathrm{cm}^{3}$, so that $a_{\mathrm{e}}\left[\mathrm{n}_{+}\right] \approx 600 \mathrm{sec}^{-1}$. Therefore, the loss of electrons by diffusion may be neglected in com-: parison with the loss by ion-electron recombination for pressures of a few torr or higher.

The first-order rate constant of electron loss by attachment can be estimated as $5 \times 10^{-12}[\mathrm{C}]+4 \times 10^{-31}[\mathrm{~B}][\mathrm{M}]$, where [B] can be taken as the NO concentration and [C] may be $\mathrm{N}_{2} \mathrm{O}$ or some other impurity that can undergo a two-body attachment reaction. Let us assume $[C] \approx 5 \times 10^{-4}[\mathrm{NO}]$, a reasonable limit for such an impurity. Substituting the values of $[\mathrm{NO}] \approx 10^{16}$ molecules $/ \mathrm{cm}^{3}$ and $[\mathrm{M}]=3.2 \times 10^{16} \mathrm{P}_{\mathrm{M}}$ molecules $/ \mathrm{cm}^{3}$, where $P_{M}$ is the total pressure in torr, gives

Rate constant of electron loss by attachment $\approx 25+150 \mathrm{P}_{\mathrm{M}} \sec ^{-1}$.

The first-order rate of electron loss by ion-electron recombination is $a_{e}\left[n_{+}\right]$, so that

Rate constant of electron loss by ion-electron recombination

$$
\approx 600 \mathrm{sec}^{-1}
$$

Thus, the estimated rate of electron loss by attachment is larger than the estimated rate of ion-electron recombination when the total pressure is greater than 4 torr. Therefore, the primary loss of charge was by ion-ion recombination, since the electrons formed negative ions (with no loss of charge) faster than they neutralized the positive ions by ionelectron recombination. 
The equilibrium electron concentration when the photolys is lamp is on is given by

$$
[e] \approx q /\left(25+150 \mathrm{P}_{M}+600\right) \approx 3 \times 10^{11 /\left(625+150 \mathrm{P}_{M}\right)} \text {, }
$$

where the rate of production of electrons is $q \approx 3 \times 10^{11}$ electrons $\left(\mathrm{cm}^{3} \mathrm{sec}\right)$. Therefore $[\mathrm{e}] \approx 1.4 \times 10^{8}$ electrons $/ \mathrm{cm}^{3}$ at $\mathrm{P}_{\mathrm{M}}=10$ torr and $[\mathrm{e}] \approx 10^{7}$ electrons $/ \mathrm{cm}^{3}$ at $\mathrm{P}_{\mathrm{M}}=200$ torr. Thus the photostationary electron concentration is lower than the negative-ion concentration of (3 to 10$) \times 10^{8}$ ions $/ \mathrm{cm}^{3}$ when the pressure is above 10 torr or so. Also, the rate of electron loss when the lamp is turned off is

$$
\frac{\mathrm{d} \cdot \ln [\mathrm{e}]}{\mathrm{dt}}=625+150 \mathrm{P}_{\mathrm{M}} \text {. }
$$

In $1 \mathrm{msec}$ after the lamp was shut off, the election conccntration would drop by a factor of 37 at. $P_{M}=20$ torr, or by a factor or more than 1000 at $P_{M}=50$ tor $r$. Therefore, under the experimental conditions used, the negative-ion concentration was larger than the electron concentration and nearly equal to the positive-ion concentration, and we could study the process of ion-ion charge exchange.

\section{Fate of the Ions Inilially Produced}

The NO' ions and the negative ions inilially furiried may hove undergone charge-exchange reactions

$$
A^{+}+F_{1} \rightleftarrows B^{+}+A
$$

and

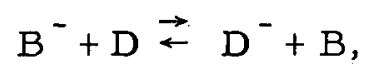

e. g. ,

$$
\mathrm{NO}^{-}+\mathrm{NO}_{2} \rightleftarrows \mathrm{NO}_{2}^{-}+\mathrm{NO}
$$

The equilibrium constants for these reactions are given by

$$
K_{B-10}=\frac{[A]\left[E^{+}\right]}{\left[A^{+}\right][E]}=\frac{\left(q_{t} q_{r} q_{v} q_{e}\right)_{A}\left(q_{t} q_{r} q_{v} q_{e}\right)_{E+}}{\left(q_{t} q_{r} q_{v} q_{e}\right)_{A}+\left(q_{t} q_{r} q_{v} q_{e}\right)_{E}} \cdot \exp \left(\Delta I / k_{B} T\right) \text {, }
$$

where $\left(\mathrm{q}_{\mathrm{t}} \mathrm{q}_{\mathrm{r}} \mathrm{q}_{\mathrm{v}} \mathrm{q}_{\mathrm{e}}\right)_{\mathrm{A}}$ represents the product of the translational, rotational, vibrational, and electronic partition functions for $A$, 
$\dot{\mathrm{k}}_{\mathrm{B}} \ddot{\mathrm{T}}=0.0257 \mathrm{eV}$, and where $\Delta \mathrm{I}=\mathrm{IP} \mathrm{P}_{\mathrm{A}}-\mathrm{IP} \mathrm{P}_{\mathrm{E}}$ is the difference in the ionization potentials of $A$ and $E$. Since $e^{\Delta I / k T}=10^{16.9(\Delta I)}$ when $\Delta . I$ is expressed in $\mathrm{eV}$, and since the $\left(\mathrm{q}_{\mathrm{t}} \mathrm{q}_{\mathrm{r}} \mathrm{q}_{\mathrm{v}} \mathrm{q}_{\mathrm{e}}\right)$ terms are nearly the same for the ion as for the neutral and thus largely cancel-out; we have

$$
\mathrm{K}_{\mathrm{B}-10} \approx 10^{16.9(\Delta \mathrm{I})} \text {. }
$$

Similar assumptions for the equilibrium expressed in Eq. (B-11) gives

$$
\mathrm{K}_{\mathrm{B}-11} \approx 10^{16.9(\triangle \mathrm{EA})}
$$

where $\triangle E A=E A_{D}-E A_{B}$ is the difference in the electron affinities, expressed in eV.

Because of the $10^{16.9}(\Delta I)$ and $10^{16.9}(\triangle E A)$ terms, in the equilibrium situation nearly all the positive ions are ions of the species with the lowest ionization potential, and the negative ions are formed from the neutral with the highest electron affinity. However, before drawing any conclusions we must also consider the kinetics of chargeexchange reartions.

A typical rate coristant for a charge-exchange reaction is the value of $\mathrm{k}=2.5 \times 10^{-11} \mathrm{~cm}^{3} / \mathrm{sec}$ for $\mathrm{O}^{+}+\mathrm{O}_{2} \rightarrow \mathrm{O}_{2}^{+}+\mathrm{O}^{19} \mathrm{Using}$ this value to get a rough estimate for the system studied gives the rate of production of any secondary positive ion from $\mathrm{NO}^{+}$as

$$
\frac{\mathrm{d}\left[\mathrm{C}^{+}\right]}{\mathrm{dt}} \approx 2.5 \times 10^{-11}[\mathrm{C}]\left[\mathrm{NO}^{+}\right] \approx 2.5 \times 10^{-11}\left(7 \times 10^{8}\right)\left(3.2 \times 10^{16}\right) \mathrm{P}_{\mathrm{C}}
$$

where $P_{C}$ is the pressure of $C$ in torr. The rate of production of $\mathrm{NO}^{+}$was around $10^{11}$ ions $/\left(\mathrm{cm}^{3} \mathrm{sec}\right.$ ) (usually more than $2 \times 10^{11}$ ), and the rate of production of $\mathrm{C}^{+}$would have been less than $10^{11}$ for $\mathrm{P}_{\mathrm{C}}<2 . \times 10^{-4}$ torr--therefore the NO was the dominant positive ion, because it seems unlikely that there would have been this high a pressure of a gas whose ionization potential is less than that of NO.

It is very likely that the negative iun was $\mathrm{NO}_{2}^{-}$rather than $\mathrm{NO}^{-}$. It has been shown that $\mathrm{NO}_{2}$ has an electron affinity greater than $3.3 \mathrm{eV}^{20}$ - - a value that almost certainly is greater than the electron affinity of any other species likely to have been present. The photolysis 
of $\mathrm{NO}$ produced $\mathrm{NO}_{2}$ and the $\mathrm{NO}_{2}$ pressure may have achieved a steady-state value of 0.2 to $2 \mu$. This is sufficiently high so that the $\mathrm{NO}_{2}^{-}$could have been the dominant negative ion, for no matter what negative ion was formed initially, it could have undergone charge exchange with the $\mathrm{NO}_{2}$ to give $\mathrm{NO}_{2}^{-}$.

Another possible reaction is ion-cluster formation,

$$
\begin{aligned}
\mathrm{NO}^{+}+\mathrm{nB} & \rightleftarrows \mathrm{NO}(\mathrm{B})_{\mathrm{n}}^{+}, \\
\mathrm{NO}_{2}^{-}+\mathrm{mC} & \rightleftarrows \mathrm{NO}_{2}(\mathrm{C})_{\mathrm{m}^{\prime}}^{-}
\end{aligned}
$$

where $B$ and $C$ are neutral gas atoms or molecules. 'This posibibility is discussed in Sec. IV, where the results of inn-mobility determinations indicate that under most conditions at least some of lie ions arc present as ion clusters. Thus, the reaction under study is the chargeneutralization reaction between $\mathrm{NO}^{+}$and $\mathrm{NO}_{2}^{-}$ions and (or) between $\mathrm{NO}^{+}$and $\mathrm{NO}_{2}^{-}$ions that are present as ion clusters. 


\section{EXPERIMENTAL APPARATUS AND PROCEDURE}

\section{A. Procedure}

The experimental problem was to measure the rate constant, $a$, for the charge-neutralization reaction

$$
\mathrm{NO}^{+}+\mathrm{NO}_{2}^{-} \stackrel{\mathrm{a}}{\longrightarrow} \text { neutral products. }
$$

The rate constant can be determined by allowing the concentration of ions to decay by means of ion recombination, and then measuring the ion concentration as a function of time. A block diagram of the apparatus is given in Fig. 1. It consisted of a vacuum ultraviolet light source whose light passed through a LiF window into the reaction cell, where it produced the ions to be studied. The light that was not absorbed in the reaction cell passed through another LiF window into an ionization cell, where the ion current served as a monitor of the lamp intensity. The reaction cell was fitted with two parallel plates and guard rings so that a voltage could be applied to collect the ions present bet.ween the plates. A lhyratron acted as a switch to apply this voltage when desired.

The experimental procedure followed during a run was to turn on the lamp, adjust it to a standard intensity, and allow the ionic concentration in the cell to come to a steady-state value. Then the lamp was turned off and simultaneously a time delay was triggered. At the end of the chosen time delay, the thyratron was fired to apply the collecting voltage to the reaction cell. The current induced by the collection of the ions passed through a resistor and the voltage that developed as a function of time on a Tektronix type 555 oscilloscope was recorded photographically. The area under the current-vs-time curve gave the value of the ion concentration at the end of the known tirre delay. This constituted one point on a concentration-vs-time curve. The experiment was repeated with different delay times until the concentration had been followed over a range such that the concentration at the longest delay time was $1 / 8$ to $1 / 30$ of the initial ion concentration. Concentrations below this point $\left[(2\right.$ to 4$) \times 10^{7}$ ions $\left./ \mathrm{cm}^{3}\right]$ were difficult to measure accurately. 


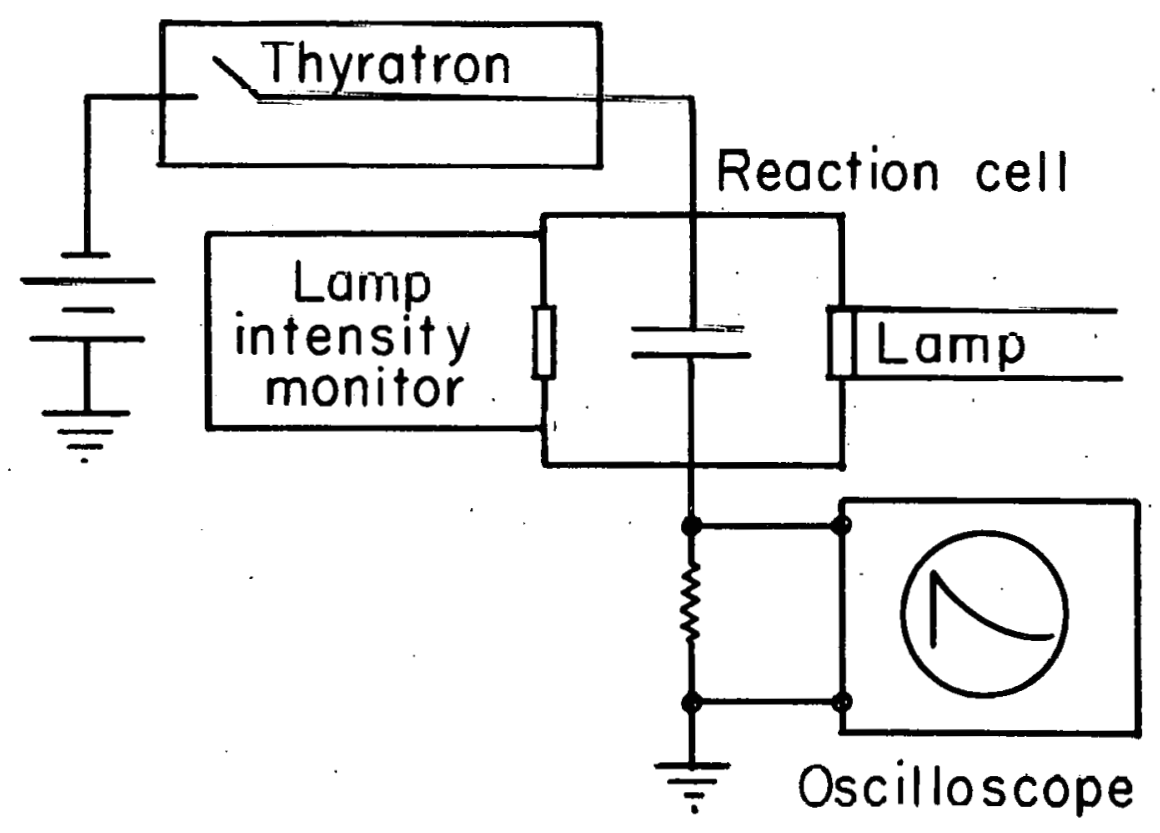

$M U-31865$

Fig. 1. Diagram of the experimental apparatus. 


\section{B. Light Source}

The light sources used were discharges excited by the $\approx 3000-\mathrm{Mc}$ microwaves produced by a QK-60 magnetron operating at a power input of 100 to 150 watts. The microwaves were coupled to a resonant cavity which contained a $25-\mathrm{mm}$ o.d. quartz tube. In most of the experiments the quartz tube was filled with pure krypton at a pressure of 1 to 2 torr. This lamp produced the $1236-\AA$ krypton resonance radiation as the primary ionizing radiation, although some $1165-\AA$ resonance radiation probably was also present. The lamp also produced considerable light of wavelengths longer than $1600 \AA$-- but this light doesn't ionize the NO. Any krypton present in the reaction cell would absorb the krypton resonánce radiation very strongly and would thus prevent the ionizing radiation from penetrating very far into the reaction cell. Therefore, for the experiments in which krypton and xenon (which contained $\approx 0.006 \%$ krypton as an impurity) were used as third-body gases, it was necessary to use another light source, and for these experiments a mixturc of liydrugen and helium was used. A reasonable intensity of ionizing radiation $\left(\approx 10^{13}\right.$ quanta/ $\mathrm{cm}^{2} \mathrm{sec}$ ) could be obtained by using about equal parts of helium and hydrogen at a total pressure around 1 to 5 tor $r$, whereas a discharge in pure hydrogen was very unstable and difficult to maintain, and a discharge in pure helium gave a much lower intensity of ionizing radiation. The spectral distribution of the $\mathrm{He}-\mathrm{H}_{2}$ lamp is not known, but the visible spectra indicated that both atomic and molecular hydrogen spectra were present, so that the ionizing radiation would consist of both the $1216 \AA$ Lyman a line and the many-lined hydrogen molecular spectra.

A thyratron circuit described by Doering ${ }^{21}$ was used to turn the lamp off and to trigger the start of the delay time. Photomultiplier :.. studies and studies done with $\mathrm{NO}$ in the reaction cell indicate that the lamp intensity drops to $1 / 2$ its initial value in about $0.2 \mathrm{msec}$ and to $1 / 10$ in about 1 risec. 


\section{Lamp Intensity Monitor}

The ionization cell used as a lamp intensity monitor was the reaction cell described by Doering and Mahan, ${ }^{17}$ which consisted of two parallel plates and guard rings in a Pyrex tube. The ionization current was produced in $80 \mu$ of $\mathrm{NO}$ and was collected by applying $90 \mathrm{~V}$ across the plates. A Keithley 610A electrometer was used to measure the current - typically $2 \times 10^{-9} \mathrm{~A}$. Since NO was used both in the lamp monitor and in the reactiun.cell, the curront prodncer in the monitor gave a good measure of the intensity of NU-ionlziny ladiation. Tho design of the apparatus had the disadvantage that only the light passing through the reaction cell, and not the direct lamp intensity, could be munilured. Thuo, any changes in light absorption in the reaction cell could not be distinguished from changes in the lamp intensity. However, repeating runs indicated that the absorption of the contents of the cell was approximately constant. The experimental setup did allow checks to be made on the purity of the third-body gases, since the ionization-cell current could be observed as the third-body gas was added to the reaction. cell.

\section{$\therefore \quad$ D. Collecting Voltage}

'the collecling-voltagc thyratron rircuit is shown in Fig. 2. After the proper time delay (as delermined by the time-delay setting on the oscilloscope), the Tektronix type 555 oscillusiope bcgan its delayed sweep and the plus gate output puts a $3 U-V$ positive pulse on the grid of the 5557 thyratron, causing it to conduct. The voltage developed across resistor $G$ or across resistors $F$ and $G$ was then applied to the high-voltage plate of the reaction cell. Before the thyratron conducted, this resistor maintained the high-voltage plate of the reaction cell at ground potential. The collecting voltage was negative in sign and was obtained from batteries (voltages of: $230 \mathrm{~V}$ or less) or from a regulated power supply $(160$ to $550 \mathrm{~V})$. The magnitude of the voltage was generally liarge enough so that the ions were collected in 1.5 to $3 \mathrm{msec}$, except at the shortest delay times where 3 to $5 \mathrm{msec}$ 


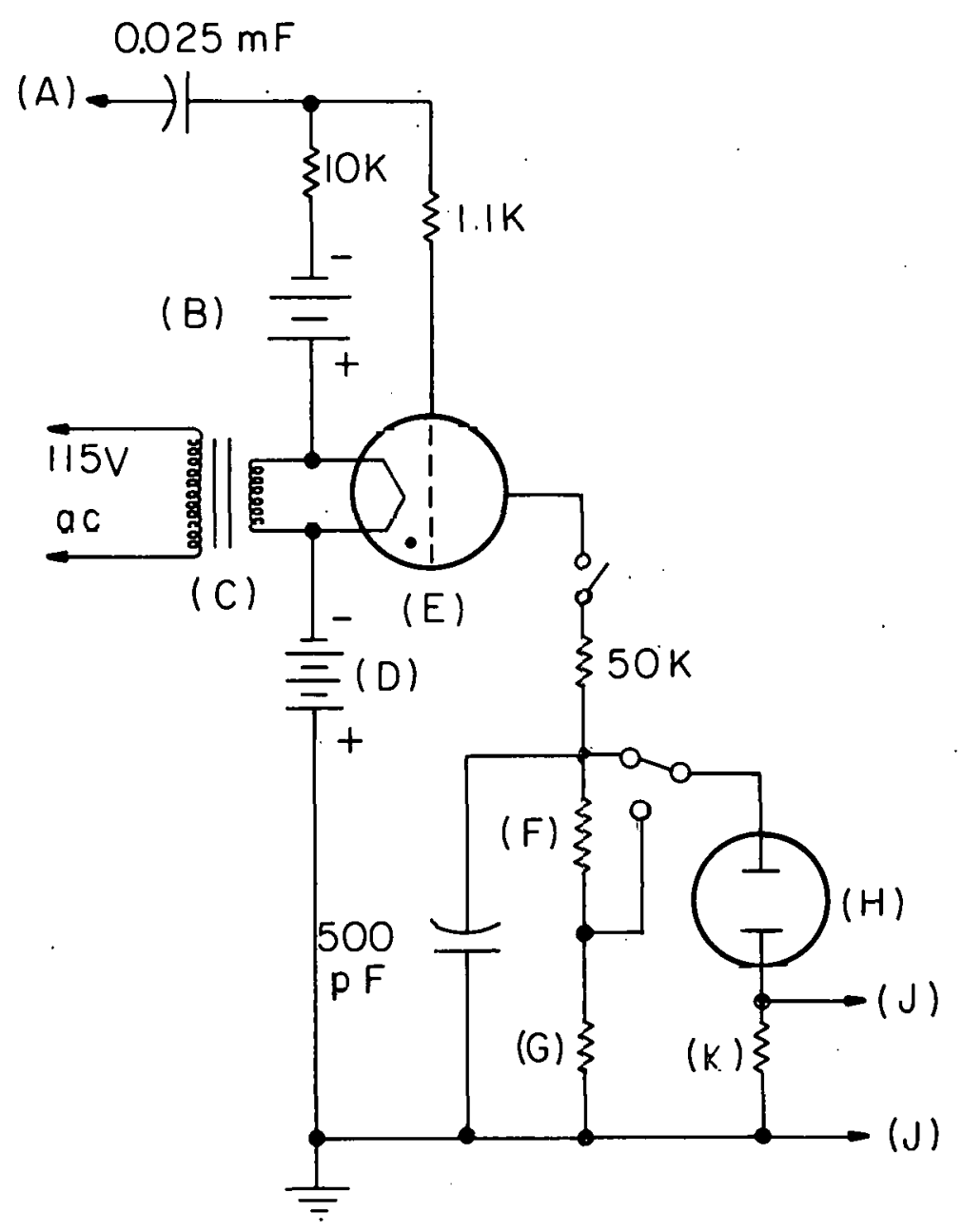

MU -31860

Fig. 2. Thyratron circuit used to turn on collecting voltage: (Resistances are shown in olims) (A) plus gate of time base $B$ of a Type 555 Tektronix oscilloscope provides a $30-\mathrm{V}$ positive pulse to fire the thyraton at the end of the delay time determined by the delayed trigger setting of the oscilloscope; (B) $7.5 \mathrm{~V}$ battery to provide negative grid bias; (C) P3062 filament transformer; (D) batteries or regulated power supply to provide the collection voltage;

$(E)$ type 5557 thyratron; (F) and (G) high-voltage collection plate is maintained at ground potential by either the $50 \mathrm{~K} \Omega$ resistor $\mathrm{G}$, or by a combination of $\mathrm{G}$ with the $50 \mathrm{~K} \Omega$ resistor $F ;(H)$ reaction cell; $(J)$ input to type $H$ and $D$ preamplifier units of oscilloscope. (K) $330 \mathrm{~K} \Omega$ resistor in parallel with the two $1 \mathrm{M} \Omega$ resistors of the ${ }^{-} \mathrm{H}$ and $\mathrm{D}$ preamplifier units transforms the ion current into a voltage. 
were required. From the results of the computer calculations described in Sec. IV, it was found to be unnecessary to correct for the number of ions recombining during the collection time. The number of ions recombining was usually less than $10 \%$ of the ions present, and the correction did not change the observed recombination coefficient by more than a few percent.

The values for the applied voltage were such that the values of $X / P$, the field intensity divided by the pressure, ranged from 0.2 to $0.7 \mathrm{~V} /(\mathrm{cm}$ torr $)$ in He, 0.4 to 4 in $\mathrm{Ar}, 0.6$ w 7 in $\mathrm{N}_{2}, 0.61,3$ in $\mathrm{Kr}$, 0.9 to $3.5 \mathrm{in} \mathrm{Xe}, 0.1$ lo $0.6 \mathrm{in} \mathrm{II}_{2}$, and 0.2 to $1.0 \mathrm{in} \mathrm{D}_{2}$. 'The higher values of $X / P$ were used at the lower pressures, since at the lower pressures the higlier ionic mohilities resulted in considerable charge separation and the resulting space charge causcd the applied voltage drops to occur mainly in the region close to the plates, so that the applied field was small in the center. Because of this, larger values of $\mathrm{X} / \mathrm{P}$ were necessary at low pressures lo collect the ions quickly. In any case, the values of $X / P$ used were so low that ion multiplication resulting from acceleration of the pusitive and negative ions would not occur, since ionization by ions is unlikely at values of $X / P$ below a few hundred volts/(cm torr). ${ }^{22}$ However, there may have been some secondary electrons emitled luring the inn-rollection process, and this may have caused surrte error in the inn concentration measurement. Any electrons emitted at the anode would be cullected quickly and would cause little external current flow. However, the clcctrons emitted at the cathode would move across to the anode and lle curront indured hy this motion would make the ion concentration appear to be larger. Also, if the value of $X / P$ were large enough, the secondary electrons might be accelerated enough to cause ionization in the gas between the plates. We can estimate an upper limit to the amount of additional ionization caused by each secondary electron by assurning that the electrons do not attach during their motion betweer the plates and by using Townsend's first-ionization coefficient, $a_{1}$, which gives the number of ion pairs produced by an electron traveling $1 \mathrm{~cm}$ in the direction of the field, so that each electron ejected at the cathode will cause 
$\exp \left(2 a_{1}\right)$ electrons to arrive at the anode, $2 \mathrm{~cm}$ away. Estimating $a_{1} / P$ to be on the order of $2 \times 10^{-4}$ ion pairs $/(\mathrm{cm} \text { torr })^{23}$ at a relatively high pressure of 500 torr gives $a_{1} \approx 0.1$ ion pairs per $\mathrm{cm}$, or $\exp \left(2 a_{1}\right) \approx 1.22$. Each secondary electron therefore would create 0.22 additional electrons in the gas, although in an actual case the attachment of some of the electrons would lower the multiplication.

It is difficult to estimate the number of secondary electrons that would be released, since (a) no experimental values of the secondary emission coefficient are available for ions in NO, (b) the values of $\mathrm{X} / \mathrm{P}$ used were lower than the range of $X / P$ in which $\gamma$, the secondaryemission coefficient, is usually measured, and ( $\ddot{c})$ the nature of the ; surface of the nickel cathode was not known. Rough extrapolations of $\gamma$ 's obtained at higher values of $X / P$ for argon ions give $\gamma$ 's from 0.002 to 0.07 electron per positive ion. ${ }^{24}$ The presence of the NO probably tends to contaminate the surface and lower the value of $\gamma$, so that we probably have $\gamma \leqslant 0.04$. If each positive ion produced 0.04 secondary electron and each secondary electron produced 0.22 more electron in the gas, there would be a total ion multiplication of about $5 \%$.

From the above discussion we see that ion mulitplication would be primarily a result of the secondary electrons ejected during ion collection. Since the estimate of $\gamma$ varied from 0.002 to 0.07 , the estimate of the ion multiplication would vary from $0.2 \%$ to $8 \%$. Because the estimate of $\gamma$ was so crude and because it was not known how $\gamma$ may have changed between experimental runs, ion multiplication was neglected. This neglect may have caused the measured ion concentrations to be a little high, but the error should be less than $5 \%$.

\section{E. Reaction Cell}

The reaction cell was a $90-\mathrm{mm}$ o.d. Pyrex tube $11.7 \mathrm{~cm}$ long with a $22-\mathrm{mm}$ i.d. window port axially located on each end. The highvoltage plate was $48 \times 58 \mathrm{~mm}^{2}$ and was $20 \mathrm{~mm}$ from the $15 \times 25 \mathrm{~mm}^{2}$ collecting plate, which was centered in an $18 \times 28-\mathrm{mm}^{2}$ hole in a $48 \times 58-\mathrm{mm}^{2}$ guard ring. The plates and guard ring were made of 
nickel and were spot-welded to tungsten wires which passed through the glass walls. The inside of the reaction cell was coated with a conducting surface of colloidal graphite (Aquadag) which was connected to an external electrode so that the walls of the cell could be maintained at ground potential. The entire reaction cell and the lamp muitor were enclosed in a grounded copper box to prevent external pickup, and coaxial cable was used for all electrical connections. An analog field plotter $^{25}$ was used to determine the equipotontial lines in the reaction rel1, and the flux lines indicated that the effective volume from which ions were collected was $14.3 \times 23.7 \times 20 \mathrm{~mm}^{3}$, or $6.77 \mathrm{~cm}^{3}$. Figure 3 shows a side view of the equipotential lines and the flux lines.

The raction sell was connected to a cunventional vacuum system; a cold trap protected the cell from mercury vapor from the manometer used to read the total gas pressure. Apiezon $W$ wax was used to seal on the two LiF windows, which were $25 \mathrm{~mm}$ in diameter and $4 \mathrm{~mm}$ thick. This wax prevented us from baking out the cell and the cell was in contact with four stopcocks which were greased either with Apiezon $\mathrm{N}$ or with Dow-Corning silicone grease. Although the system was pumped down to ( 1 to 2$) \times 10^{-6}$ torr before each experiment, the prossirre in the closed-off reaction cell built up to $(0.2$ to 1$) \times 10^{-4}$ torr. Althnugh some of this gas may have been stopcock grease vapor, most of it was probably absorbed air in the grease, or absorbed gas from the previuus filling of the reactinn rell.

\section{F. Gas Purity}

Cylinder nitric oxide was further purified by repeated distillation from an isopentane slush bath at $-160^{\circ} \mathrm{C}$, and by pumping on the NO when it was cooled to $-196^{\circ} \mathrm{C}$. Table I gives the impurities found by mass-spectral analysis of the various samples of NO used, and of the $\mathrm{N}_{2}, \mathrm{Kr}, \mathrm{Xe}, \mathrm{Ar}, \mathrm{He}, \mathrm{H}_{2}$, and $\mathrm{D}_{2}$ used as third-body gases. Also included is the least impurity that could have been detecled in each mass-spectral run. In the case of $\mathrm{Ar}, \mathrm{He}, \mathrm{H}_{2}$, and $\mathrm{D}_{2}$, it was possible to determine upper limits to $\mathrm{H}_{2} \mathrm{O}$ impurity at levels below the limit of 


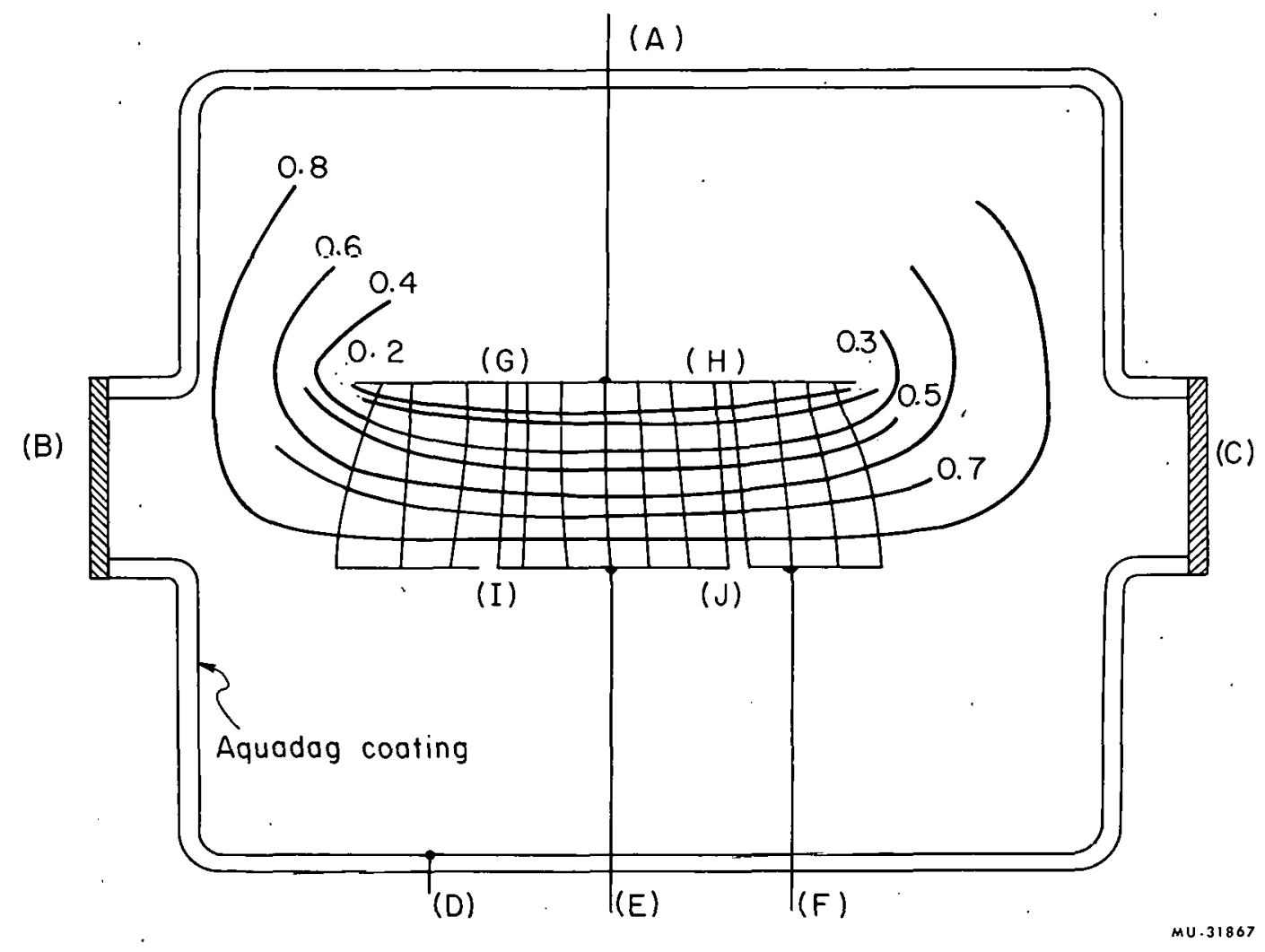

Fig. 3. Side view of equipotential surfaces and flux lines in reaction cell: (A) high-voltage terminal; (B) LiF window to lamp; (C) LiF window to lamp-intensity monitor; (D) Aquadag-coating terminal; (E) collectingplate terminal; ( F) Guard-ring terminal; (GHIJ) cross section of actual collection volumc. 
Table I. Impurities found ir gases used.

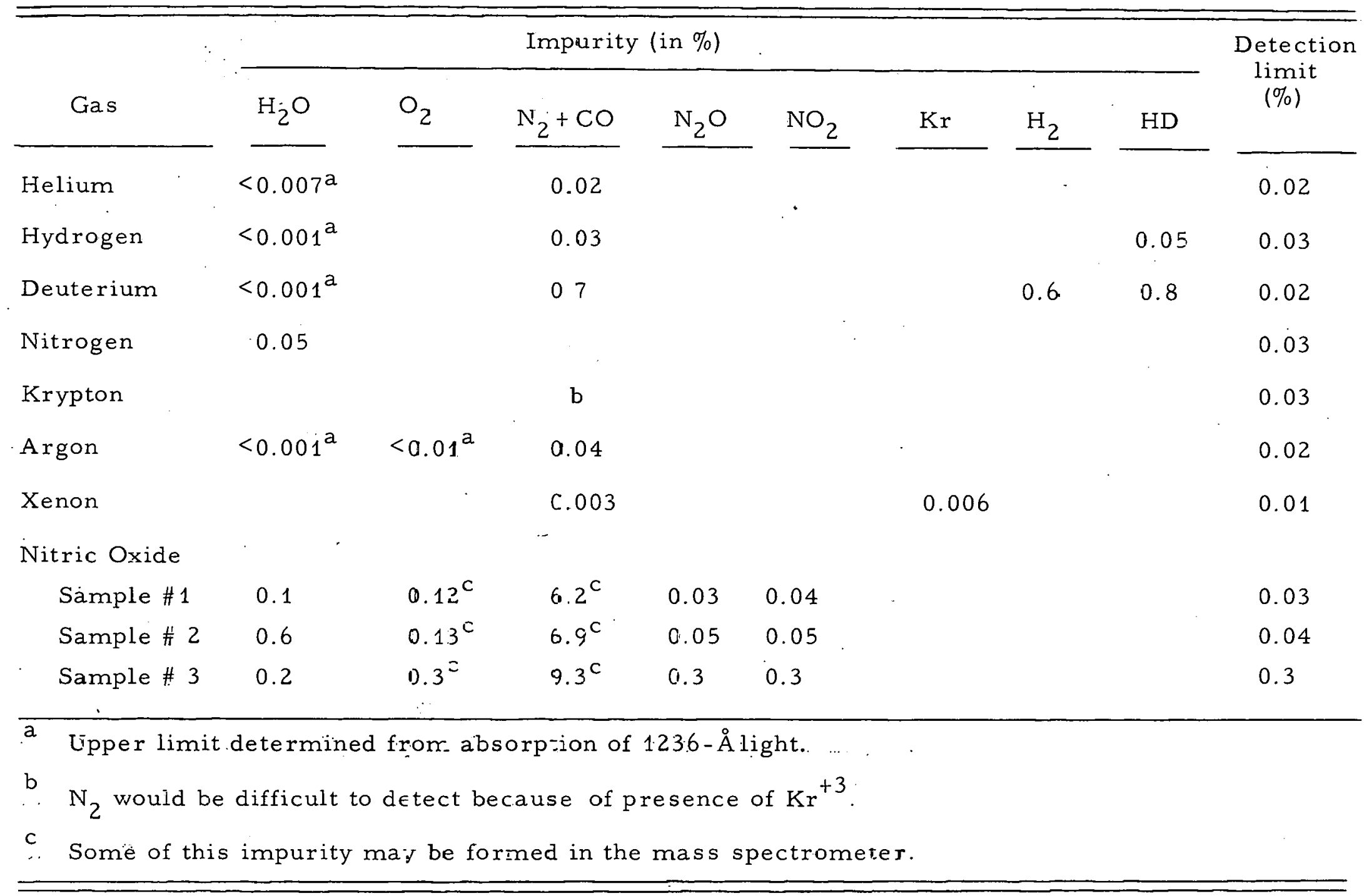


mass-spectral sensitivity by observing the absorption of the $1236-\AA$ radiation and by using the known absorption coefficient for $\mathrm{H}_{2} \mathrm{O}$.

\section{G. Experimental Difficulties}

Because of the capacitance of the reaction cell and of the coaxial : cable between the cell and the oscilloscope, and the input capacitance of the oscilloscope, a charging current flowed when the voltage was applied to the cell. A correction for this charging current was determined by making a blank run in which the voltage was applied but. the lamp was not turned on. Such a blank run was made for each regular run with the lamp on, and the ion concentration was determined by measuring the area between the curve for the current due to ions plus charging and the curve due to capacitor charging alone.

Another difficulty encountered was that the oscilloscope trace did not always return to zero--instead, it would sometimes return to a value of around $2 \%$ of the maximum deflection and then remain almost constant for several msec although it continued to slowly retuin tu zero. Figure 4 shows. (a) an example of a run without the "tail, "and (b) an example of a run with the tail. This tail was more obvious when the pressure was low, and it was also more serious for the light thirdbody iga:se:s:- - which suggests that it may have been due to ions diffusing into the collection volume from the region outside. Indeed, it was found that the ions within the collection volume were collected more rapidly than were the ions that the guard ring collected, and the guard ring still collected ions for several msec after the collecting plate had stopped collecting. Some of these ions that were outside the normal collecting volume may have diffused into the collecting volume.

Because of the resistance in the oscilloscope input circuit, the collecting plate was a few tenths of a volt from ground potential during the time of ion collection. Therefore, a resistor was placed so that the current collected by the guard ring and by the Aquadag coating also developed a small voltage, so that the collecting plate and the guard ring were at nearly the same potential during the time of ion collection. However, since the ions in the collection volume were collected before 
(a)

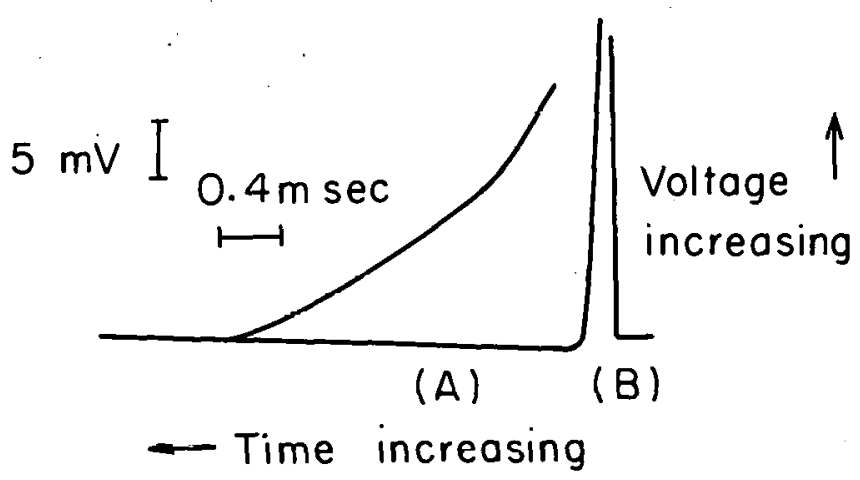

(b)

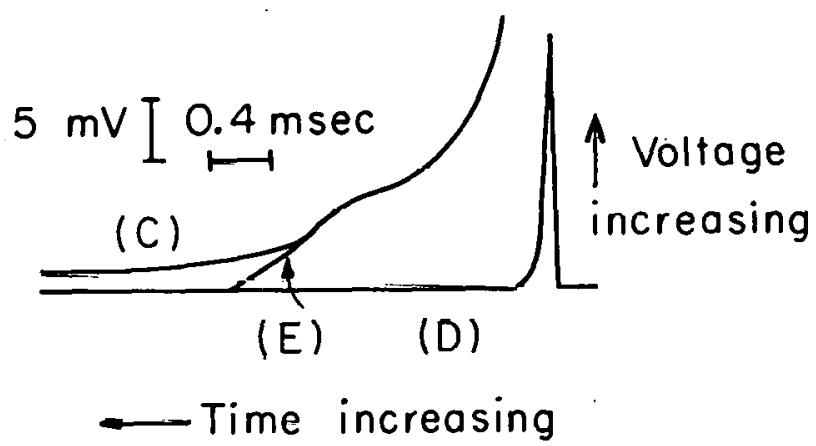

$M U .31868$

Fig. 4. (a) Illustration of an experimental curve without a Tail. (A) Blank run showing capacitance charging current (which gocs off scale on this trace). (B) Time when voltage is applied to cell. Experimental run is NO-Kr at a total pressure of 250 torr.

(b) Illustration of the extrapolation procedure and an experimental curve with a tail: (C) tail of run whose peak voltage (not shown here) was $80 \mathrm{mV}$; (D) blank run; (E) extrapolated curve; the tail beyond this curve is not included in the determination of the total charge collected. Experimental run is NO-He at a total pressure of 92 torr. 
the ion current to the guard ring was zero, there was a small potential difference $(\approx 0.1 \mathrm{~V})$ which slightly. favored the collector plate. Also, the concentration gradient in the cell after the ions in the collection volume had been collected favored diffusion into the collection volume. In order to evaluate the area under the current-time curve the collection current was extrapolated to zero so as to not include the tail--as illustrated in Fig. 4(b). However, this procedure did count the few ions that may have drifted into the collection volume during the time of ion collection. The upper limit of the number of ions that may have drifted into the collection volume during the regular collection time can beiroughly :estimated by assuming that the number of ions drifting in was the same for the entire collection time as it was at the start of the tail. This puts an upper limit on the number of extra ions collected at 10 to $20 \%$ of the total ions for the low-pressure runs with tails.

Another question is whether or not contact potentials were present, and if so, what their effect was. In an effort to study this, some runs were done in which a bias battery was used to apply about $0.1 \mathrm{~V}$ to one of the plates, so that ions might be collected during the delay time before the collecting voltage was applied. The result was that at long delay times the ion concentration was about 2 to 5\% lower-hardly more than the normal experimental scatter. Also, some calculations were carried out (using the program described in Sec. IV) on the effect of a small voltage of about $0.1 \mathrm{~V}$. The results of these .. calculations indicate that such a voltage would cause the measured value of the recombination coefficient to be 1 to $3 \%$ high, and in view of the other experimental uncertainties, it is not necessary to correct for any contact potentials that may have been present. 


\section{IV: ESTIMATION OF ION MOBILITIES}

Since the mobility of gaseous ions depends upon the mass and size of the ions, we attempted to estimate the ionic mobilities so that x/e would have a better idea of the nature of the ions involved. The estimation was carried out by comparing the experimental currenttime curves produced during ion collection with calculated currenttime curves. Here, in Sec. IV, we describe the calculation of these curves and the results of the ionic mobility estirriation.

\section{A. Description of the Method of Computation}

When the cullecting voltage is applier to the reaction cell, the ions present between the plates begin to move in lhe applied electric field and thus inducc a current whose time behavior is recorded on an oscillogram. The shape of the current-vs-time curve is determined by a number of factors: (a) the ionic concentration at the time the voltage is applied, (b) the number of ions lost by recombination or diffusion during the time of collection, (c) the initial distribution of the ions, (d.) the magnitude of the applied voltage, (e) the distance between the plates, and (f) the mobilities of the various ions present. All of these are known in principle except the exacl foim of tho ion diatrihution and the ion mobilities. We attempled to match the observer current-time curves with calculated curves, using the ion mobility as a. parameter.

The current, $i$, induced in the external circuit by the motion of a concentration of $\mathrm{n}_{+}$singly charged positive ions and a concentration of $\mathrm{n}$ - singly charged negative ions distributed along the distance $y$ between the cathode at $y=0$ and the anode at $y=d$ is given by 26,27

$$
i=\frac{e A}{d} \int_{0}^{d}\left(n_{+}\left|v_{+}\right|+n_{-}\left|v_{-}\right|\right) d y
$$

where $\left|v_{+}\right|$and $\left|v_{-}\right|$are the magnitudes of the velocities of the positive and negative ions, $e$ is the electronic charge, and $A$ is the area of the collecting plate. 
If the electric field is not too large, then the magnitudes of the ion velocities are related to the electric field, $X$, by

$$
\left|\mathrm{v}_{+}\right|=\mathrm{k}_{+}|\mathrm{x}|
$$

and

$$
\left|v_{-}\right|=k_{-}|x|
$$

where $k_{+}$and $k_{-}$are the ionic mobilities of the positive and negative ions. Substituting Eqs: $(D-2)$ and $(D-3)$ into Eq. $(D-1)$ gives

$$
i=\frac{e A}{d} \int_{0}^{d}|x|\left(n_{+} k_{+}+n_{-} k_{-}\right) d y .
$$

Poisson's equation states

$$
\frac{\partial X}{\partial y}=4 \pi e\left(n_{+}-n_{-}\right)=\frac{\partial^{2} V}{\partial y^{2}},
$$

where $V$ is the voltage and the permittivity is taken as $1 /(4 \pi)$.

Since the ionic concentration at any point is a function of the ion-current flow at that point and the loss by ion recombination, continuity requirements give

$$
\frac{-\partial n_{+}}{\partial t}=-\frac{\partial\left(n_{+} k_{+} x\right)}{\partial y}+a n_{+} n_{-}
$$

and

$$
\frac{\partial n_{-}}{\partial t}=+\frac{\partial\left(n_{-} k_{-} X\right)}{\partial y}+a n_{+} n_{-},
$$

where $t$ is the time and $a$ is the second-order rate constant for ion recombination. Tho boundary conditions are

and

$$
\mathrm{n}_{+}(\mathrm{d}, \mathrm{t})=0
$$

$$
\mathrm{n}_{-}(0, \mathrm{t})=0
$$

By using Eqs: (D-4) through (D-9) it is possible to evaluate the induced 
current as a function of time if the initial conditions $X(y, 0), n_{+}(y, 0)$ and $n_{-}(y, 0)$ are known, and if $a, k_{+}, k_{-}$, and $v_{0}$, the applied voltage, are known. To simplify the problem somewhat, we replaced $k_{+}$and

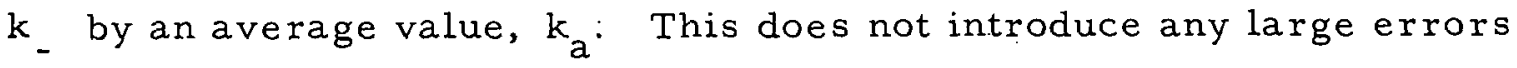
because (a) the iuns probably do have mobilities thal differ by les 3 than $50 \%$, and (b) if one ion has a larger mobility, the action of the spacecharge effect is such that the slower ion moves in a larger field than the faster'ion, so that the net result is similar to both ions' moving with the average mobility. Also, we assumed that only one type of ion was present and that the nature of the ions did not change during the period of ion collection.

Tt. is convenient to introduce the reduced parameters 27

$$
\begin{gathered}
P_{+}=n_{+} / n_{0}, \quad P_{-}=n_{-} / n_{0}, \quad W=V / V_{0}, \\
U=X d / V_{0}, \\
I=d i /\left(A k_{a} n_{0} e V_{0}\right),
\end{gathered}
$$

and $\quad \xi=\mathrm{y} / \mathrm{d}, \quad \because \tau=\mathrm{k}_{\mathrm{a}} \mathrm{V}_{\mathrm{o}} \mathrm{t} / \mathrm{d}^{2}$.

$$
\Omega=a d^{2} n_{0} /\left(k_{a} V_{0}\right), \quad G=4 \pi n_{0} e d^{2} / V_{0}
$$

where $n_{0}$ is the ion concentration when the collecting voltage is initially applied, $V_{0}^{\cdot}$ is the voltage applied, $n_{+}$and $n_{-}$are the concentrations of positive and negative ions and $\mathrm{k}_{\mathrm{a}}$ is the ion mobility. In terms of these reduced parameters, Eqs. (D-4) through (D-9) become

$$
\begin{gathered}
I(\tau)=\int_{0}^{1}\left(P_{+}+P_{-}\right) U d \xi \\
\left.U=\frac{\partial W}{\partial \xi} ; \frac{\partial^{2} W}{\partial \xi^{2}}=\frac{\partial U}{\partial \xi}=G_{+} P_{+}\right), \\
\frac{-\partial P_{+}}{\partial \tau_{+}}=\frac{-\partial\left(P_{+} U\right)}{\partial \xi}+\Omega P_{+} P_{-}
\end{gathered}
$$




$$
\begin{gathered}
\frac{-\partial P_{-}}{\partial \tau}=\frac{\partial\left(P_{-} U\right)}{\partial \xi}+\Omega P_{+} P_{-}, \\
P_{+}(1, \tau)=0,
\end{gathered}
$$

and

$$
\dot{P}(0, \tau)=0
$$

- In order to set the problem up for solution on an IBM 7090 digital computer the differential Eqs. $(D-14)$ and $(D-15)$ were replaced by the following difference equations:

$$
\begin{gathered}
P_{+}\left(\xi_{i}, \tau_{j+1}\right)=P_{+}\left(\xi_{i}, \tau_{j}\right) \\
+\Delta \tau\left\{( \frac { 1 } { \Delta \xi } ) \left[P_{+}\left(\xi_{i+1}, \tau_{j}\right) U\left(\xi_{i+1}, \tau_{j}\right)\right.\right. \\
\left.\left.-P_{+}\left(\xi_{i}, \tau_{j}\right) U\left(\xi_{i}, \tau_{j}\right)\right]-\Omega P_{+}\left(\xi_{i}, \tau_{j}\right) P_{-}\left(\xi_{i}, \tau_{j}\right)\right\},
\end{gathered}
$$

and

$$
\begin{aligned}
& P_{-}\left(\xi_{i}, \tau_{j+1}\right)=P_{-}\left(\xi_{i}, \tau_{j}\right)+\Delta \tau\left\{( \frac { 1 } { \Delta \xi } ) \left[P_{-}\left(\xi_{i-1}, \tau_{j}\right) U\left(\xi_{i-1}, \tau_{j}\right)\right.\right. \\
& \left.\left.-P_{-}\left(\xi_{i}, \tau_{j}\right) U\left(\xi_{i}, \tau_{j}\right)\right]-\Omega P_{+}\left(\xi_{i}, \tau_{j}\right) P_{-}\left(\xi_{i}, \tau_{j}\right)\right\},
\end{aligned}
$$

where $\xi_{i}=i \Delta \xi, \quad \xi$ going from $\xi=0$ at the cathode to $\xi=1$ at the anode, and $\tau_{j}=j \Delta \tau$. By use of Eqs. $(D-18)$ and $(D, 19)$ the reduced ion concentrations at each point $\left(P_{+}, P_{-}\right)$are calculated for the later reduced time $\tau_{j+1}=\tau_{j}+\Delta \tau$ from the values of $P_{+}, P_{-}$, and $U$ at the reduced time $\tau_{j}$. Then: Eq? $(D-13)$ is integrated once to give U plus an integration constant, and this result is integrated again and the integration constant is evaluated from 


$$
\int_{0}^{1} U d \xi=W(1, \tau)=1
$$

since

$$
\mathrm{V}(\mathrm{d}, \mathrm{t})=\mathrm{V}_{0}
$$

In this way, the values of $U\left(\xi_{i}, \tau_{j+1}\right)$ are found and the whole procedure is repeated with the values of $P_{+}, P_{-}$, and $U$ at reduced time $\tau_{j+1}$ to give the values at $\tau_{j+2}$, etc. At various intervals, Eq. $(D-12)$ is integrated to give the reduced current, I, as a function of the reduced time, $\tau$. The actual number of ions collected at the anode was also calculated, and the total amount of charge collected was nearly equal to the tutal charge induced by the motion of the ions (if there were no ion recombination, the charges would be exactly equal ); however, the time behavior of the induced ion current differs from the time behavior of the collected ion current.

The complete FORTRAN-II computer program is given in Appendix A.

\section{B. Results of Computer Caluclations}

Because of the resistance and capacitance in the circuit that applies the collecting voltage, the actual voltage between the cathode and anode is given by

$$
\mathrm{V}=\mathrm{V}_{0}\left[1-\exp \left(-\mathrm{t} / \mathrm{T}_{\mathrm{RC}}\right)\right]
$$

where $\mathrm{T}_{\mathrm{RC}}$ is the $\mathrm{RC}$ constant for the circuit and was either $1.8 \times 10^{-5} \mathrm{sec}$ or $2.3 \times 10^{-5} \mathrm{sec}$ in the apparatus used. The reduced value of $T_{R C}$ is ${ }^{\prime} R C$, where.

$$
\tau_{\mathrm{RC}}=\mathrm{k}_{\mathrm{a}} \mathrm{V}_{0} \mathrm{~T}_{\mathrm{RC}} / \mathrm{d}^{2}
$$

Since the experiments, were done with different values of $k_{a} V_{0}$, some compromise had to be made in the choice of ${ }^{\tau} \mathrm{RC} \cdot$. 
In most of the experiments the values of $\tau_{R C}$ were between 0.005 and 0.05 , with values around 0.02 being more common. A calculation was done with three different values of $\tau_{\mathrm{RC}}$, and Fig. 5 shows the results for $\tau_{\mathrm{RC}}=0.0035,0.0175$, and 0.0875 . In the rest of the calculations, $\tau_{R C}=0.0175$ was used.

Since the exact form of the initial distribution is not known, a preliminary calculation was done using three different initial distributions. In one case the initial distribution was assumed to be $n_{+}(y, 0)=n_{-}(y, 0)(\pi / 2) n_{0} \sin \pi(y / d)$, which would be the distribution in the limiting case in which diffusion to the plates is the only mechanism for ion loss during the delay time after the lamp is turned off. In the other extreme (no diffusion), the distribution would be uniform right up the the plates (with the concentration zero at the surface of the plates). This distribution was also used as a test case. A more realistic semiuniform distribution was also used in which the ion concentration is uniform in the center, but smaller in the neighborhood of the plates; it is shown in Fig. 6(a). Figure 6(b) shows the current-time curves obtained for three distributions. The semiuniform and the uniform distributions give nearly identical results, but the sine distribution gives a somewhat different result. In the remaining calculations both the sine and the semiuniform initial distributions were used.

Figure 7 shows a comparison of calculated curves with points obtained from an experimental oscillogram, indicating the general agreement. However, it is rather tedious to make such a: plot, since various values of $k_{a}$ must be assumed in order to convert $i$ to $I$. and $t$ to $\tau$, and also several measurements of $i$ and $t$ are necessary. In order to make mobility estimates with less effort, another procedure was used. This procedure is to estimate the mobility from the length of time required for ion collection. The time at which ion collection actually ceases is poorly defined, since the current goes to zero asymptotically. However, it is possible to extrapolate a nearly linear section of the curve so that each calculated curve is characterized by an extrapolated reduced ion collection time, $T_{C}$. The extrapolation is illustrated in Figs. 8 and 9 for two different values of $G$ (the shapes of the reduced current-reduced time curves depend mainly on the value of $G)$. 


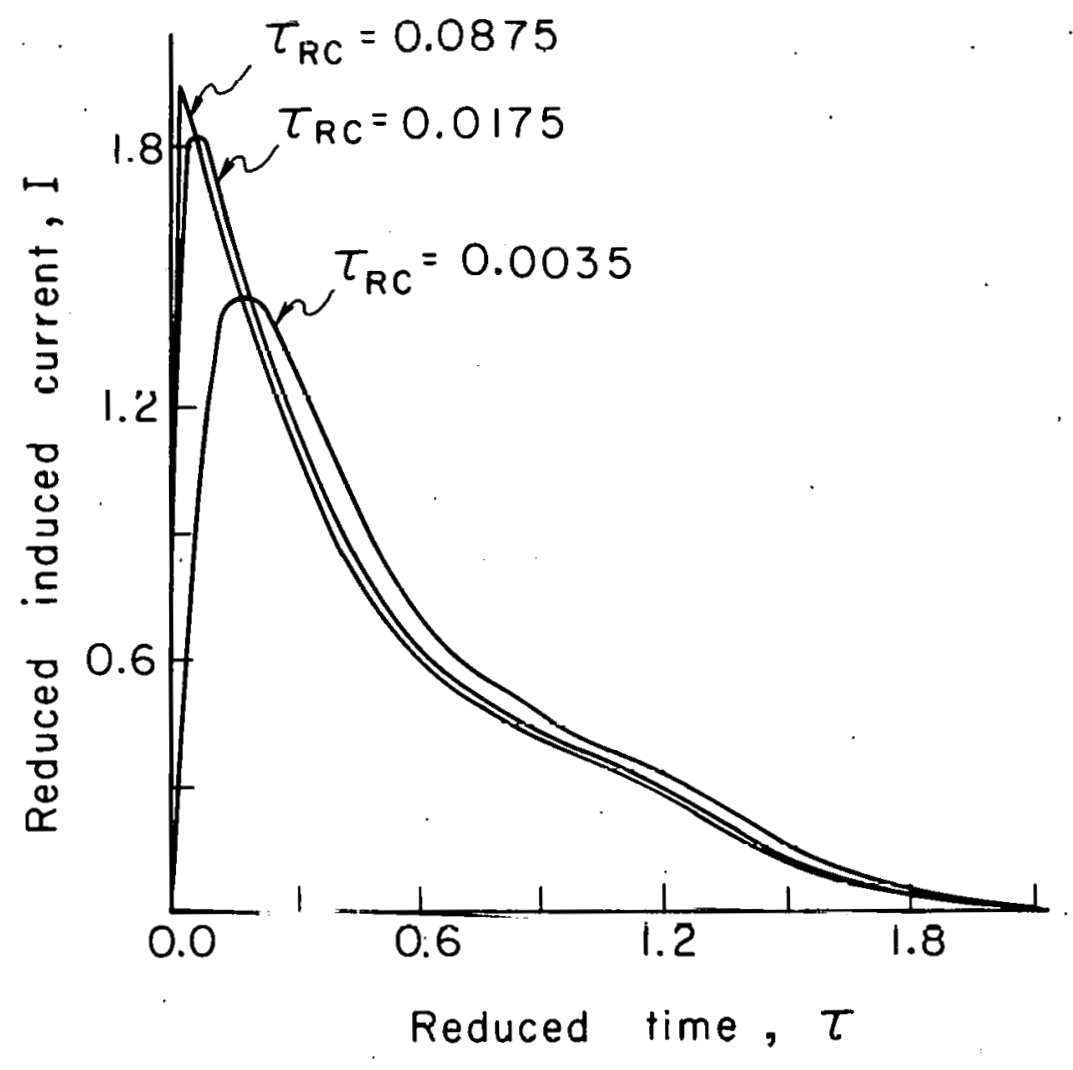

MU. 31869

Fig. 5. Reduced-current reduced-time curves for three different values of $\tau_{\mathrm{RC}}$. All curves have $\mathrm{G}=7.2426$, $\Omega=0$, and a semiuniform initial distribution. 


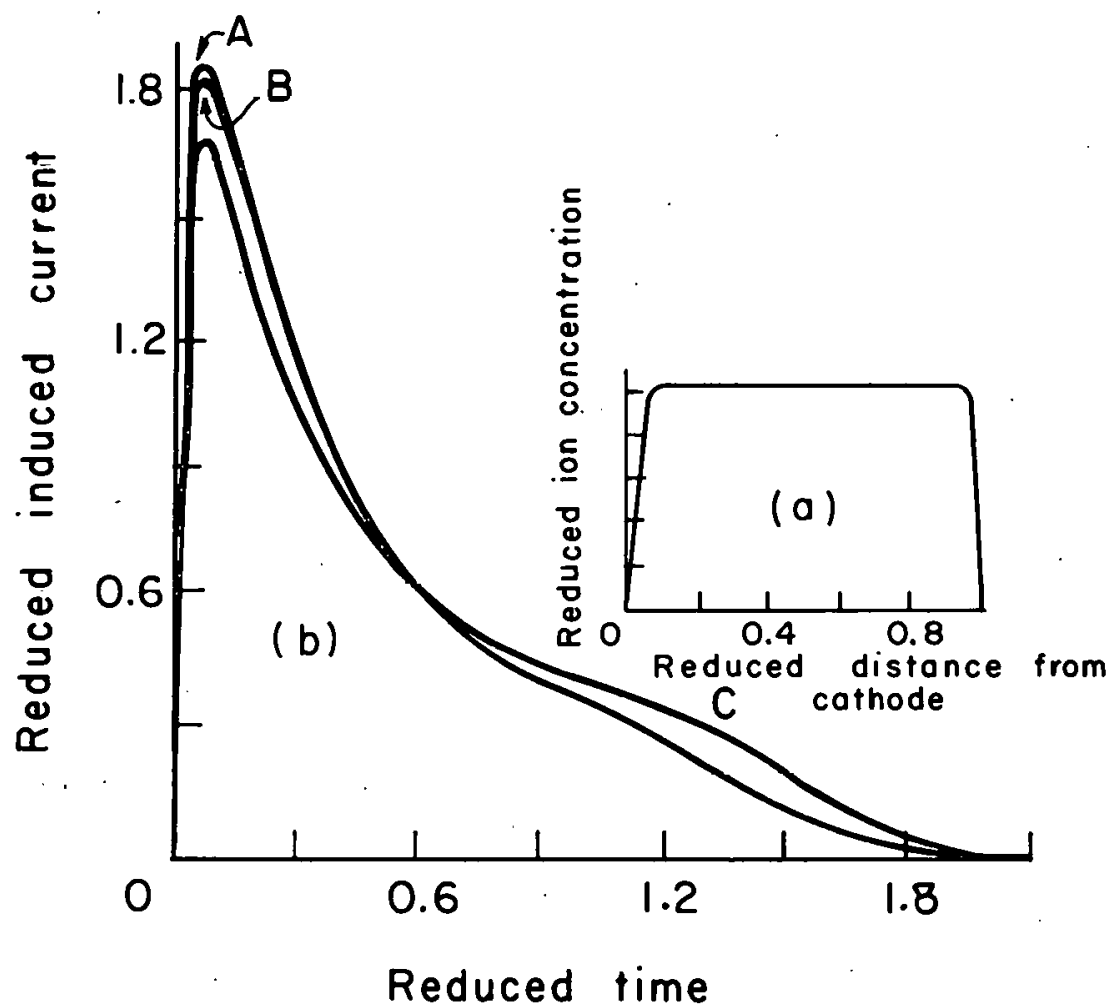

$M U-31870$

Fig. 6(a). Semiuniforn initial distribution.

(b) Reduced-current reduced-time curves using different initial distributions: curve (A) uniform initial distribution, curve (B) semiuniform initial distribution, curve (C) Sine initial distribution.

All curves are for $\mathrm{G}=7.2426, \Omega=0$, and $\tau_{\mathrm{RC}}=0.0175$. 


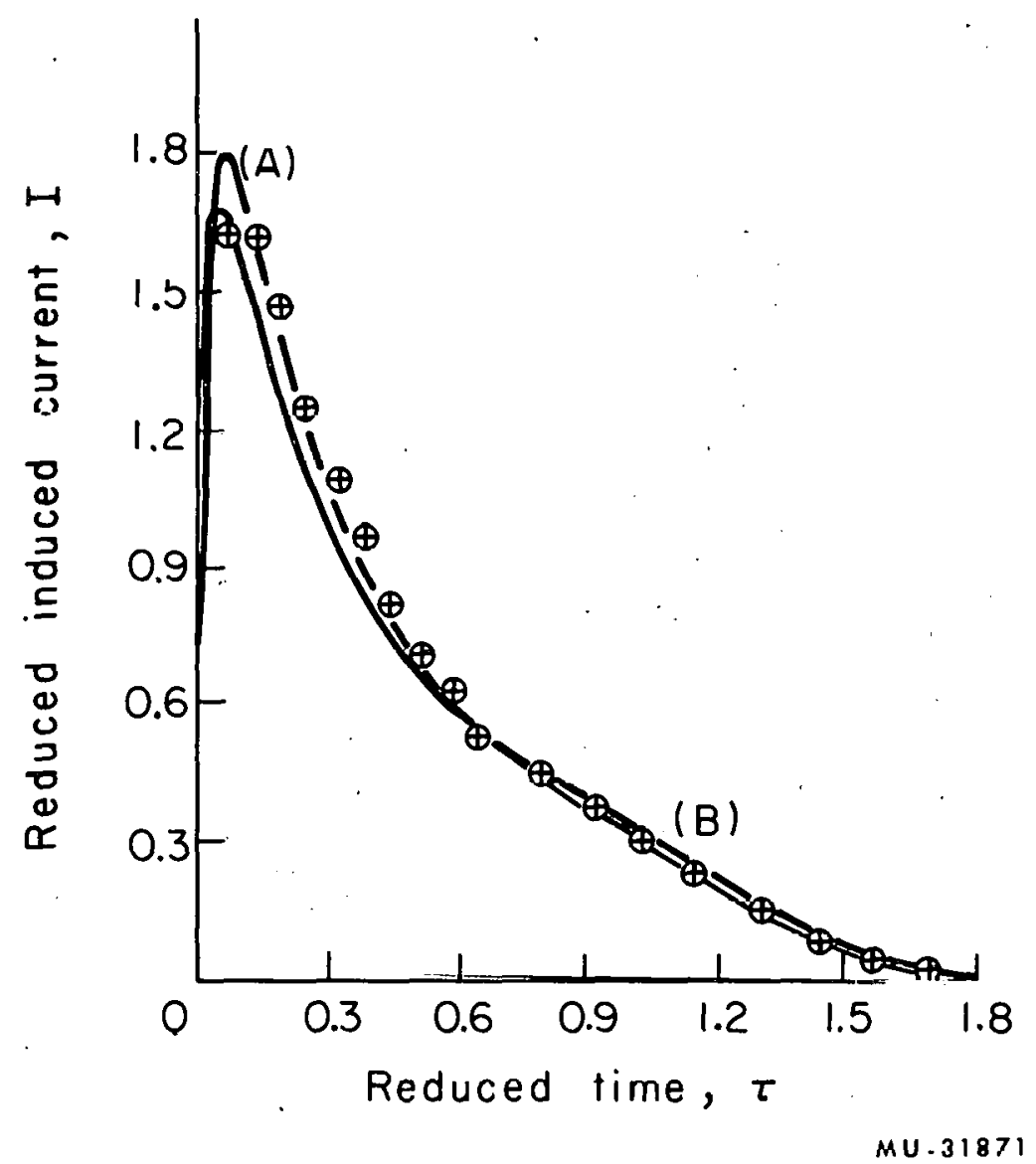

Fig. 7. Comparison of points from experimcntal currenttime curve with calculated curves. The assumed value of the mobility is $9.75 \mathrm{~cm}^{2} /$ (volt sec) and the experimental run is NO-Kr at 138 torr, $V_{0}=270 \mathrm{~V} ; n_{0}=2.4 \times 10^{8}$ ions $/ \mathrm{cm}^{3}$, $a=0.75 \times 10^{-6} \mathrm{~cm}^{3} / \mathrm{sec}$, the value of $G$ experimental is 6.44 and $\Omega$ experimental is 0.27 , while the calculation uses $G=6.3$ and $\Omega=0.30$. Curve (A) Semiuniform initial distribution. Curve (B) Sine initial distribution. 


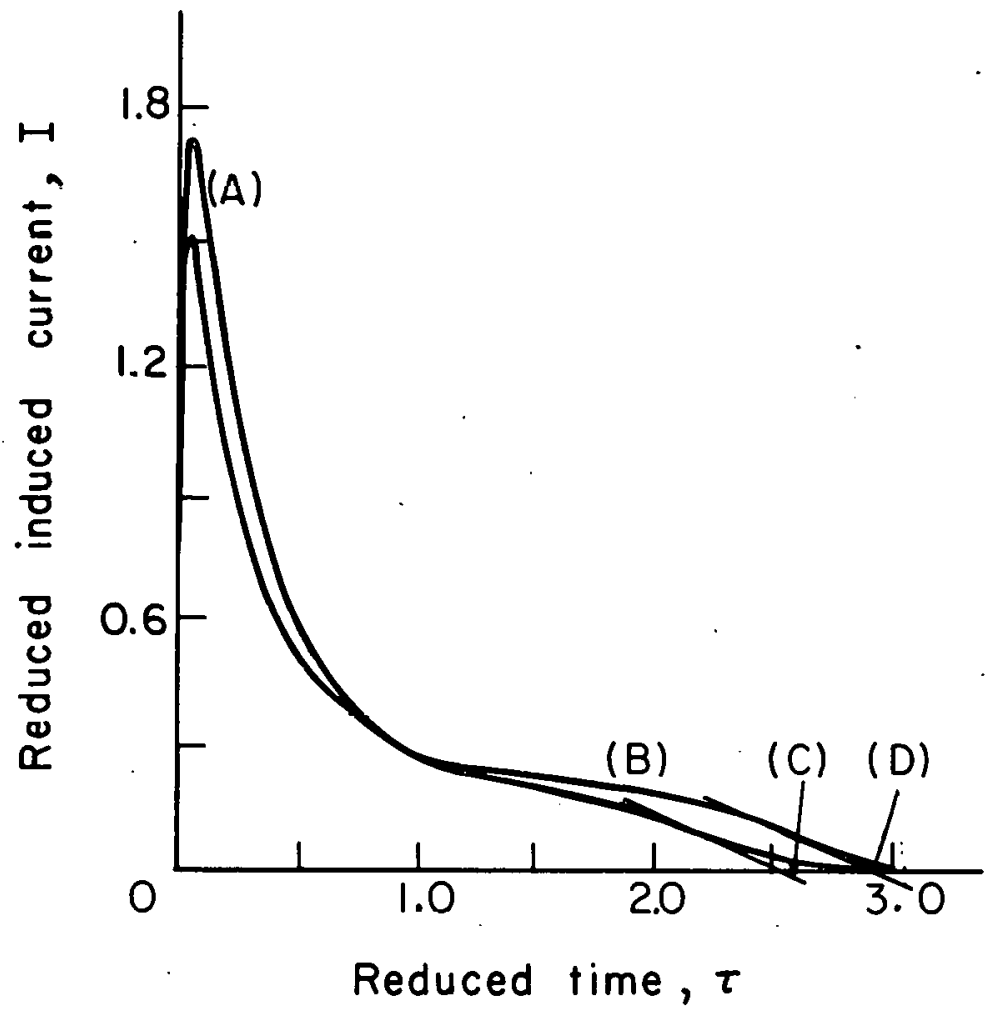

$M U-31872$

Fig. 8. Current-time curves showing the determination of the reduced extrapolated ion-collection time, $\mathrm{T}_{\mathrm{C}}$. Both curves have $G=16, \Omega=0.10, \tau_{R C}=0.0175$. Curve $(\AA)$ has the semiuniform initial distribution and gives the $\mathrm{T}_{C}=2.53$ shown at C. Curve (B) has the sine initial distribution and gives the $\mathrm{T}_{\mathrm{C}}=2.91$ shown at (D). 


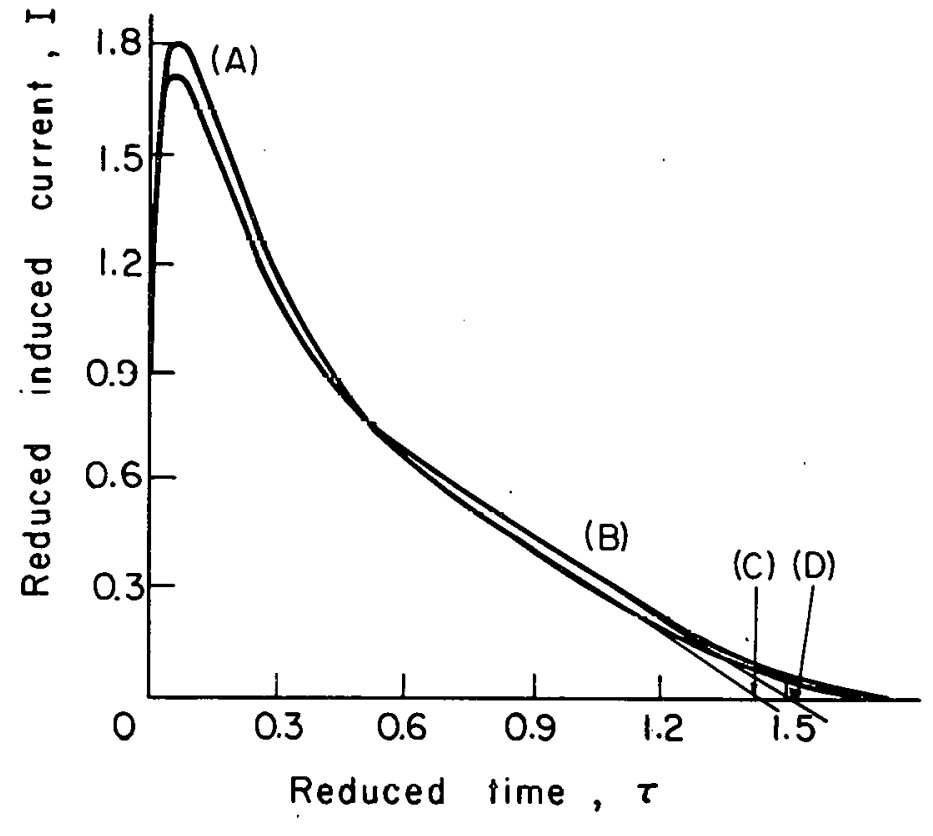

$M \cup .31873$

Fig. 9. Current-time curves showing reduced extrapolated ioncollection time, $\mathrm{T}_{\mathrm{C}}$, determination. Both curves have $\mathrm{G}=5, \Omega=0.10$, and $\tau_{\mathrm{RC}}=0.0175$. Curve (A) has the semiurifosm initial distribution and gives the $\mathrm{T}_{\mathrm{C}}=1.42$ shown at (C). Curve (B) has the sine initial distribution ard gives the $\mathrm{T}_{\mathrm{C}}=1.49$ shown at $(\mathrm{D})$. 
The values of $T_{C}$ were obtained from calculations using various values of $G$ and $\Omega$ for both the sine and the semiuniform initial distribution. These values are tabulated in Table II along with the fraction of ions actually measured, ${ }_{n} / n_{0}$ (as determined from the area under the induced current-time curves). Also in Táble II are the values of $G_{\dot{C}}$, defined as

$$
G_{C}=n_{C} G / n_{0}=4 \pi n_{C} e^{2} / V_{0} \text {. }
$$

The values of $G_{C}$ are included because the direct experimental measurement gives ${ }^{n} \mathrm{C}$ rather than $\mathrm{n}_{0}$. There is an uncertainty of $2 \%$.or $3 \%$ in the values in Table II caused by errors from the finite step size used in the integration. There is an additional error of 2 to $4 \%$ in the extrapolation procedure, as well as an additional uncertainty when comparing cases having different values of $\tau_{R C}$ with the values calculated for $\tau_{R C}=0.0175$. Figure 10 shows that the values of $T_{C}$ plotted against $G_{C}$ give approximately linear plots for each value of $\Omega$ and for each initial distribution. For small values of $G_{C}$, the curves are quite close, but the differences increase as $G_{C}$ increases.

The method of evaluating the ion mobility is to calculate $G_{C}$ from Eq. (D-23) and estimate $\Omega$ from Eq. (D-11) by using an estimated value of $k_{a}$. Then $T_{C}$ for these values of $G_{C}$ and $k_{a}$ is determined by interpolating between the curves in Fig. 10. Two values of $T_{C}$ are "... generally obtained, one from each initial distribution. Then the experimental extrapolated ion-collection time, ${ }^{t} \mathrm{C}$, is measured and the mobility is found from Eq. (D-11) to be

$$
k_{a}=d^{2} T_{C} /\left(v_{0} t_{C}\right) \text {. }
$$

This value of the mobility is used to revise the estimate of $\Omega$, and the process is repeated. The accuracy of this procedure is probably not better than $10 \%$, and there is often a considerable difference between the mobilities obtained by using the two different initial distributions. When the mobilities are different, the choice of the actual mobility can be based on the recognition that the different mobilities are limiting results and that, as the mobility increases, the true mobility approaches the mobility obtained from the sine initial distribution. The actual 
Table IT. Values determincd for $\mathrm{T}_{\mathrm{C}}$ and $\mathrm{n}_{\mathrm{C}} / \mathrm{n}_{0}{ }^{a}$

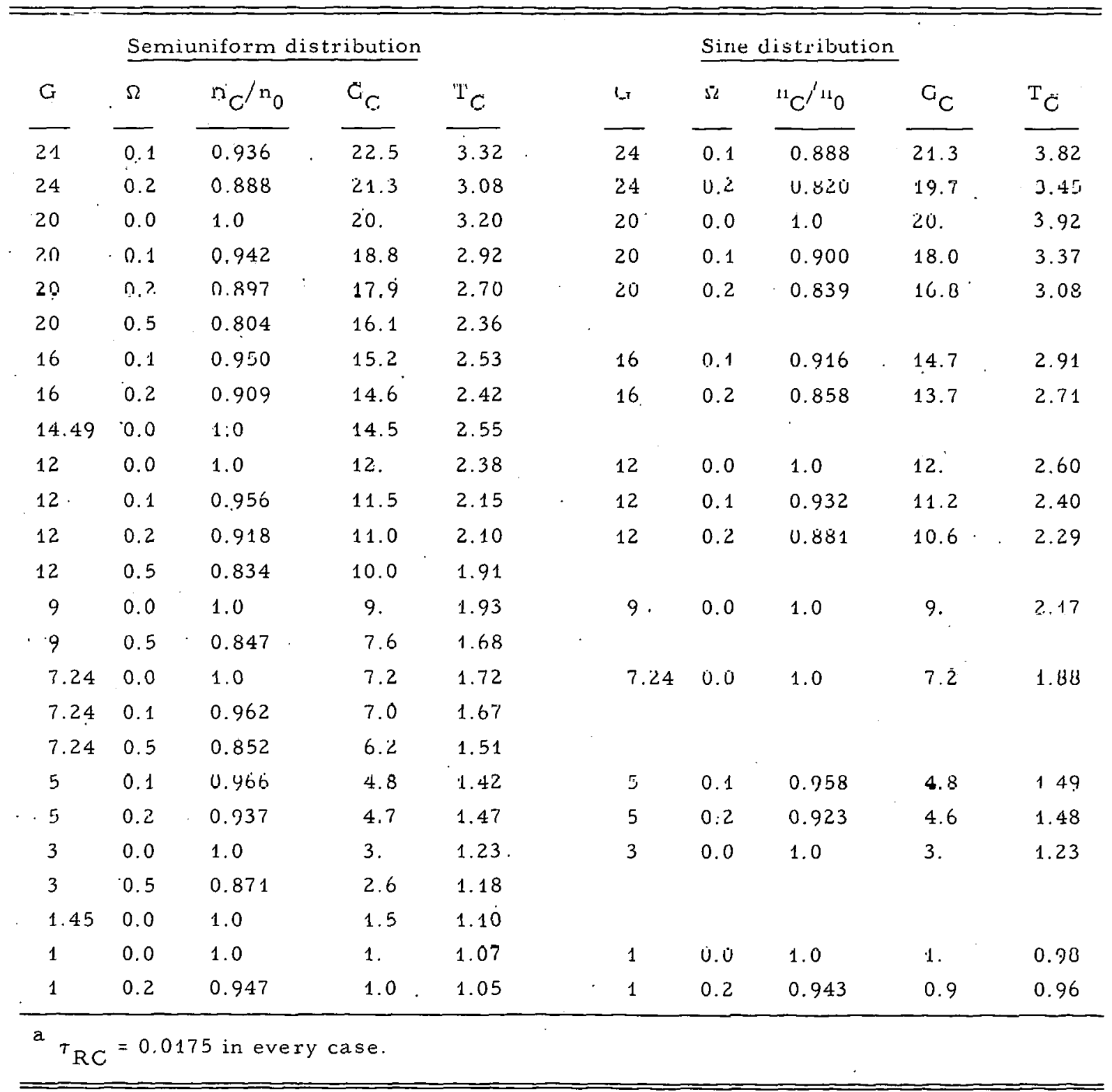




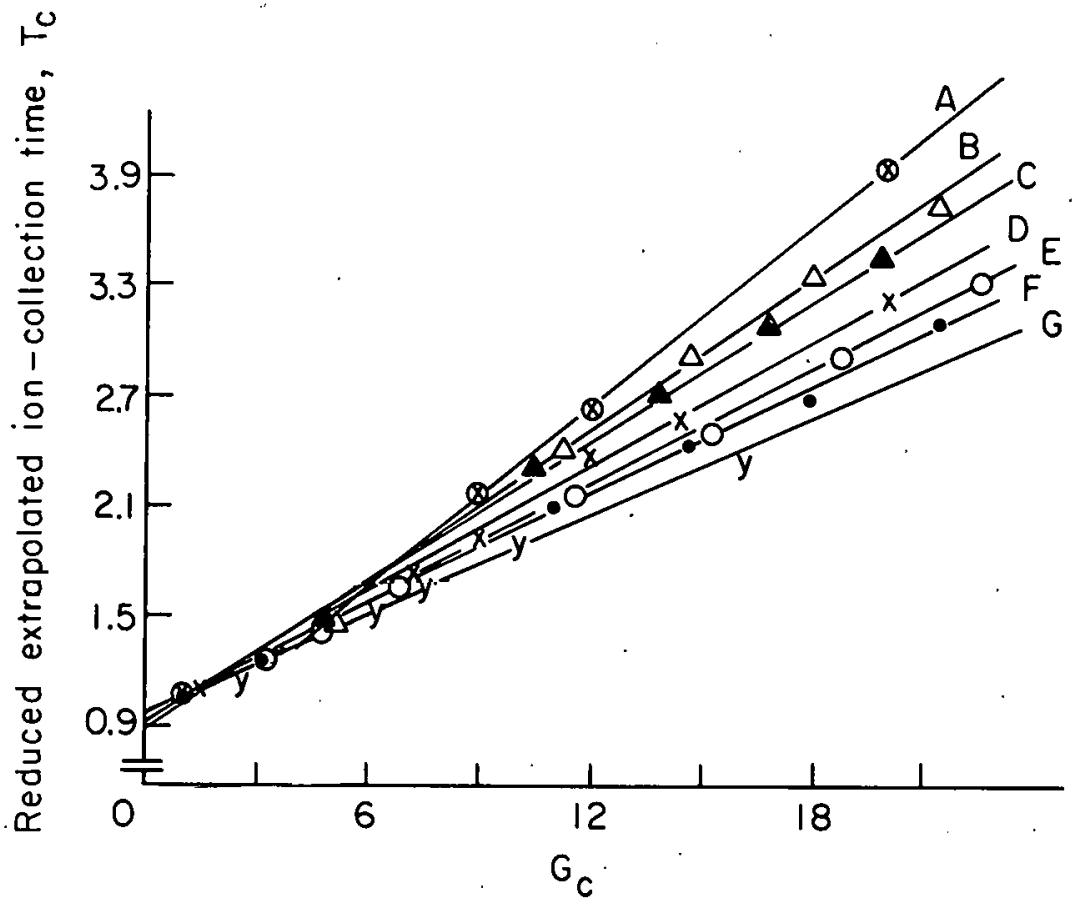

MU -31874

Fig. 10. Reduced extrapolated ion-collection time as a function of $\mathrm{G}_{\mathrm{C}}$. (A), (B), and (C) have initial sine distributions with $\Omega=0$ for (A), $\Omega=0.1$ for (B), and $\Omega=0.2$ for (C). (D), (E), (F), and (G) have semiuniform initial distributions with $\Omega=0$ for (D), $\Omega=0.1$ for $(E), \Omega=0.2$ for $(F)$, and $\Omega \doteq 0.5$ for $(G)$. 
choice made was such that for $\mathrm{k}_{\mathrm{a}}=30 \mathrm{~cm}^{2} /(\mathrm{V} \mathrm{sec})$ the mobility is $\mathrm{k}_{\mathrm{a}}=(1 / 3) \mathrm{k}_{\mathrm{aS}}+(2 / 3) \mathrm{k}_{\mathrm{aS}-\mathrm{U}}$, where $\mathrm{k}_{\mathrm{aS}}$ is the mobility determined by using the sine distribution and $\mathrm{k} a S-\mathrm{U}$ is from the semiuniform distribution; for $k_{a}=60$ the mobility is $k_{a}=(2 / 3) k_{a S}+(1 / 3) k_{a S-U}$, etc. The mobilities were determined for three or four delay times for each experimental run and these values were averaged. Then the value of the mobility at 1 atm pressure was calculated from

$$
k_{760}=k_{a}(P / 760),
$$

whese $\Gamma$ io tho gas pressure in torr. The experiments were done at room temperature, so that the mobilities lisled in Table III are at 760 torr and approximately $298^{\circ} \mathrm{K}$.

Because the calculated eurrent-time surves do not match the observed curves exactly, there is always an uncertainty in the determination of $k_{a}$. The method described above places greater emphasis on matching the curves in the region near the end of the collection period. This may not be the best method, but it was used for lack of knowledge of a better method.

From these calculations we can estimate what fraction of the total ions present when the voltage is applied are aclually measured. Figure 11 shruws a plot of $n_{C} / n_{0}$ as a function of $G_{C}$ forvarious values of $\Omega$ for the two initial distributions. By use of these curves it is estimated that the ion loss by recombination during the time of ion. collection inliuduces an crror of only a few percent, and that the corrected values of the rate coirstant for rocombination, $a$, are generally within the experimental uncertainty in the determination of $a$.

\section{Relation Between the Mobility and the Nature of the Ions:}

In order to discuss the results given in Table III, we necd a theory to relate the ionic mobility to the nature of the ions. One such theory has been derived by Langevin ${ }^{28}$ and recalculatcd by Hasse 29 In this theory, the interaction potential bctween the ions and the neutrals is assurned to be an ion-induced dipole potcntial with a hard-sphere core: 
Table III. Experimental determinations of the ion mobility at 760 torr, approx $298^{\circ} \mathrm{K}$

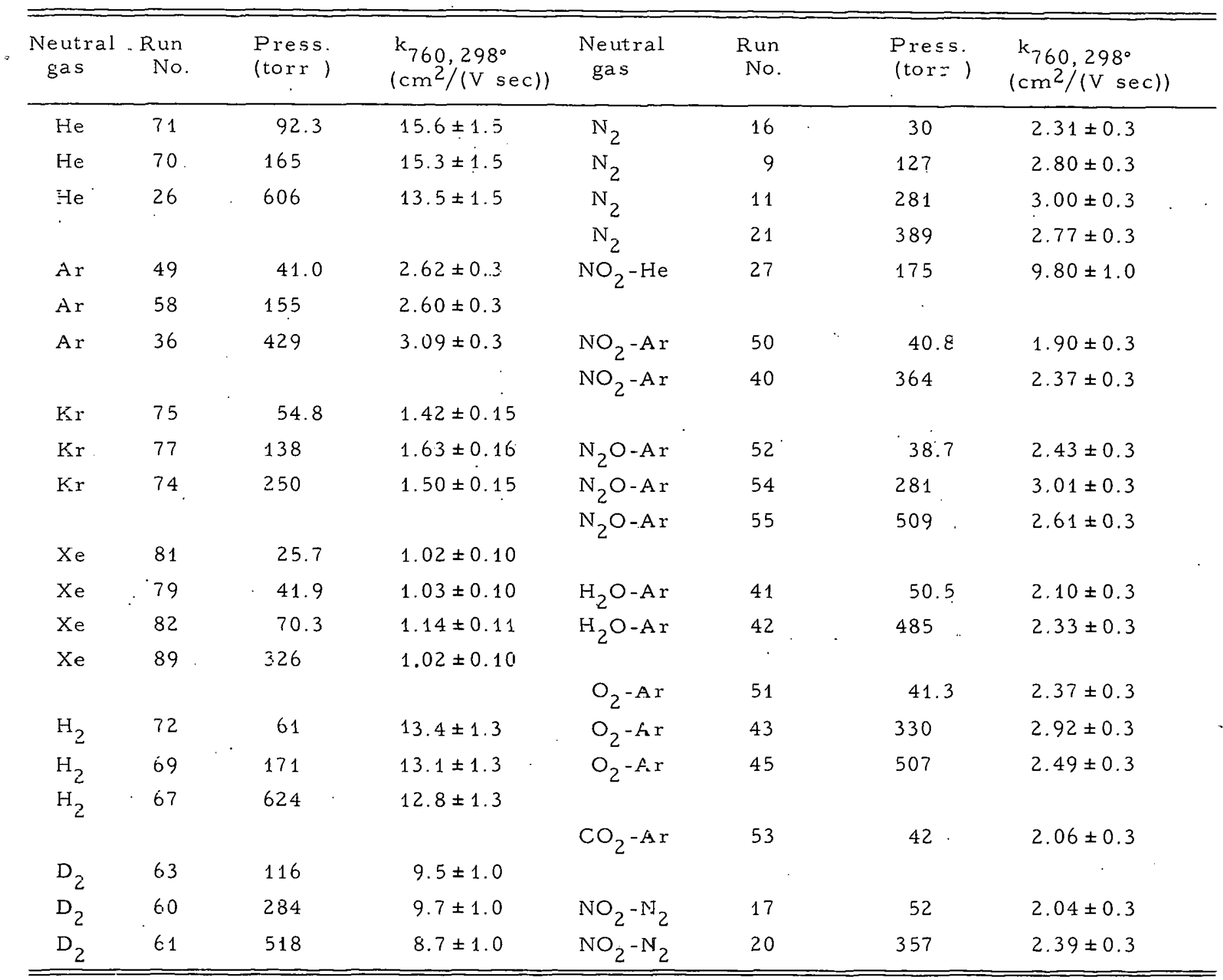




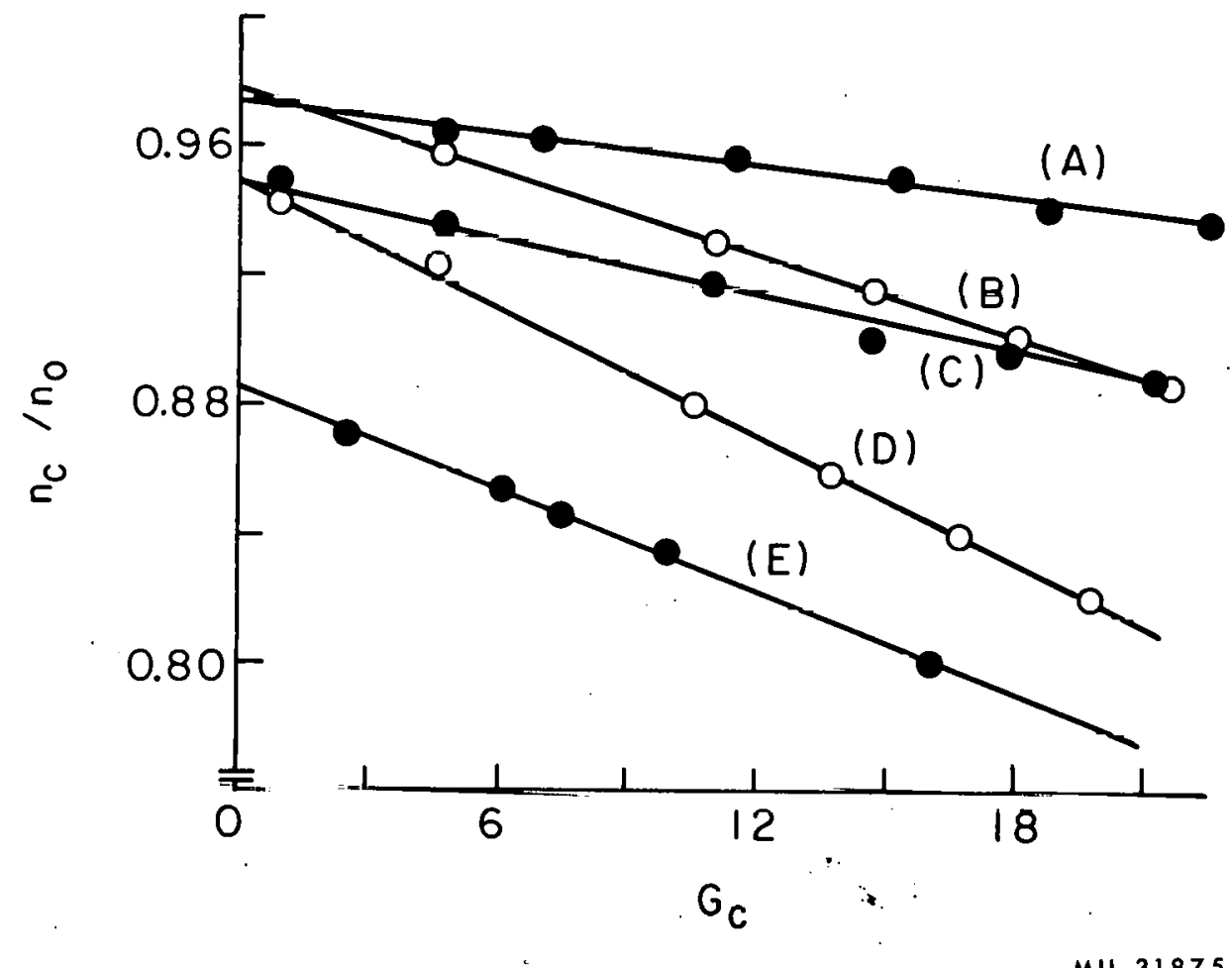

Fig. 11. Fraction of ions actually measured as a function of $\mathrm{G}_{\mathrm{C}}$. (A), (C), and (E) have semiuniform initial distributions with $\Omega=0.1$ for (A), $\Omega=0.2$ for (C), and $\Omega=0.5$ for (E). (B) and (D) have sine initial distributions with $\Omega=0.1$ for (B) and $\Omega=0.2$ for (D). 


$$
\begin{aligned}
& V(r)=-a_{M} e^{2} /\left(2 r^{4}\right) \quad \text { for } r>s, \\
& V(r)=\infty: \text { for } r \leqslant s,
\end{aligned}
$$

where $a_{M}$ is the polarizability of the neutral, e. is the electronic charge, and the hard-sphere distance is defined as

$$
\mathrm{s}=\left(\mathrm{s}_{\mathrm{I}}+\mathrm{s}_{\mathrm{M}}\right) / 2
$$

where $s_{I}$ and $s_{M}$ are the hard-sphere diameters for the ion and the neutral. is 30

When this potential is used the expression for the ionic mobility

$$
k=\frac{0.462 A}{P(273 / T)\left[4 \pi\left(2.689 \times 10^{19}\right) a_{M^{\mu}}{ }^{1 / 2}\right.}
$$

where $P$ is the gas pressure in atmospheres, $T$ is the absolute temperature, $\mu=M_{M} M_{I} /\left(M_{I}+M_{M}\right)$ is the reduced mass in gram-molecular weight units, and $A$ is tabulated 29,30 as a function of a parameter, $\lambda$, defined as

$$
\lambda=\left[2 \mathrm{k}_{\mathrm{B}} \mathrm{Ts}^{4} /\left(\mathrm{a}_{\mathrm{M}} \mathrm{e}^{2}\right)\right]^{1 / 2}
$$

where $k_{B}$ is the Boltzman constant and $s$ is defined in Eq. (D-27). This theory then predicts that the mobility,depends both upon the masses and upon the sizes of the ions and the neutrals, as. well as on the polarizability of the neutrals. In view of the rough determination of the mobilities, this theory should be sufficiently realistic for our purposes, therefore it is used because of its simplicity in comparison with more detailed calculations.

For one of the parameters of the theory it is required that the hard-sphere diameters of the ion and the neutral be estimated. The procedure used was to take values derived from Goldschmidt's values of the distance between ionic and molecular centers on impact ${ }^{31}$ as lower limits, use Lennard-Jones $\sigma$ values ${ }^{32}$ as upper limits. Table IV gives the $s$ values and the $\lambda$ values from Eq. (D-29). The different ions are assumed to be $\mathrm{NO}^{+}$and $\mathrm{NO}_{2}^{-}$ions with varying amounts of ion clustering. The values of $s$ for the ion clusters were estimated from a comparison with values for various polyatomic molecules. 
Table IV. Values ${ }^{\circ} \equiv \mathrm{s}$ and $\lambda$ for different ion sizes in various neutral gases. ${ }^{a}$

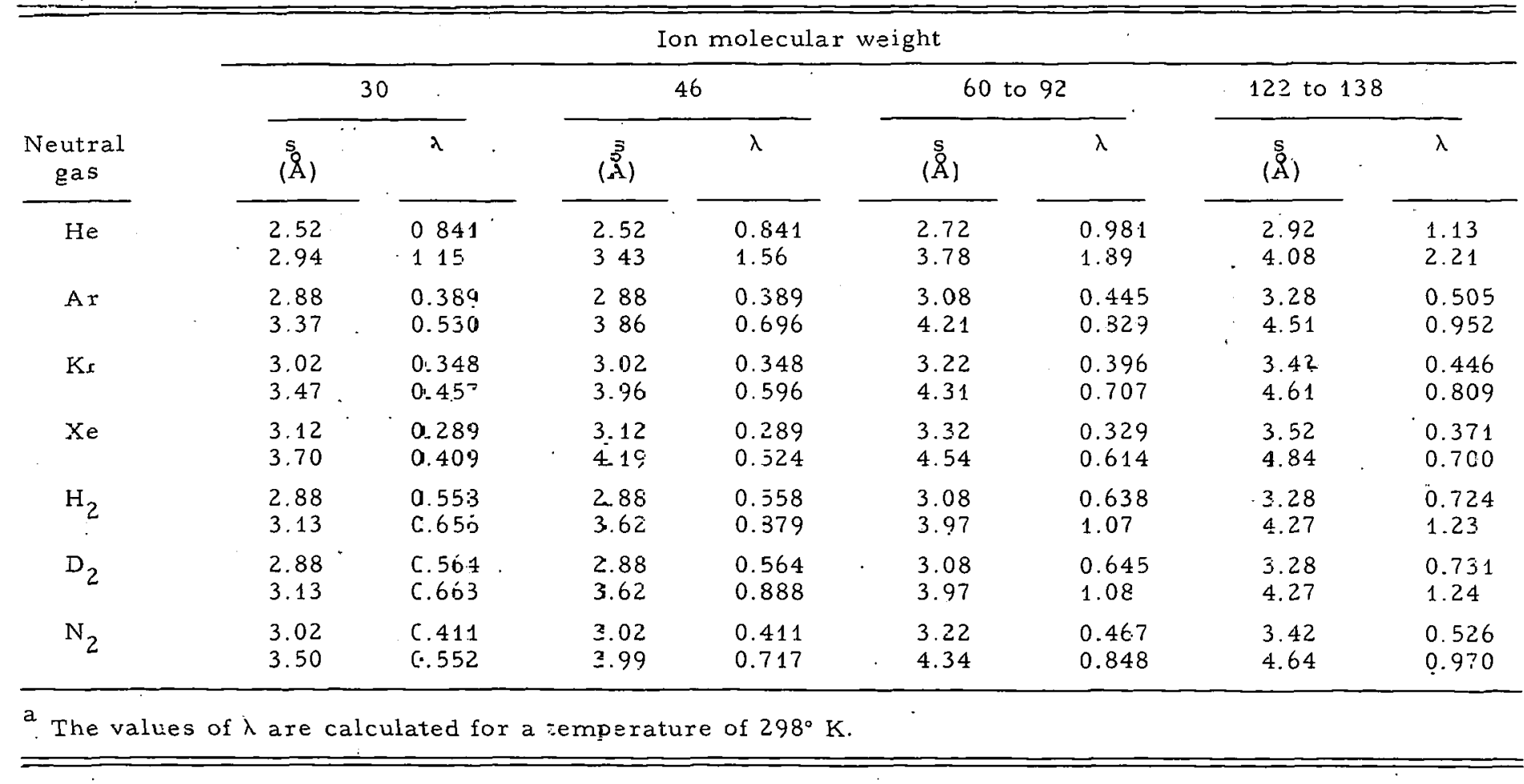


The predicted mobilities listed in Table $\mathrm{V}$ may be likened to the experimental determinations of the mobilities of alkali ions. ${ }^{33}$ The predicted values in $\mathrm{Ar}, \mathrm{Kr}, \mathrm{Xe}$, and $\mathrm{H}_{2}$ agree with the experimental results to within 2 to $4 \%$ (the predicted values are usually lower than the experimental values). The predicted values of the mobilities in He agree better with the observed mobilities of molecular ions ${ }^{34}$ and are lower than the experimental mobilities of the alkali ions in $\mathrm{He}$, indicating that the alkali ions have somewhat smaller values of $s$ than are used in this calculation. However, the predicted mobilities in $\mathrm{N}_{2}$ are abnut 10\% highor than line experimental values of the alkali ion mobilities in $\mathrm{N}_{2}$. Since the mobility in $\mathrm{N}_{2}$ is not very sensitive to our choice of $\mathrm{s}$, this prediction indicates that some failure of the theory causes the predicted mobilities to be too high for ions in $\mathrm{N}_{2} \cdot 35$ For this reason, Table $\mathrm{V}$ also includes values of the mobility in $\mathrm{N}_{2}$, which. were obtained from a comparison with mobilities of alkali ions in $\mathrm{N}_{2} \cdot 33$

\section{Discussion of the Results of the Mobility Determination}

By comparing the experimental values of the mobility listed in Table III with the predicted values given in Table V, we can get some idea of the nature of the ions involved. In the NO-He mixtures, the predicted mobility depends mainly on the size of the ion, and the experimental results would be consistent with an ion molecular weight anywhere from 40 to 150 . When 5 to $8 \mu$ of $\mathrm{NO}_{2}$ is added to the $\mathrm{NO}-\mathrm{He}$ mixture the mobility is considerably lower and indicates an ion whose hard-sphere diameter is larger than $4.1 \AA$.

In NO-Ar mixtures the high-pressure result indicates an ion molecular weight of less than 30 , but the other pressures indicate the ion molecular weight to be between 46 and 60 . The smaller ion at high pressure is a strange result (assuming that the discrepancy is real and not just a result of the rough mobility determination), since one would expection-cluster formation to be more likely as the pressure increases. Perhaps there were some electrons present during this run and they caused the average mobility to increase. Upon addition of several microns of $\mathrm{NO}_{2}$ to the NO-Ar mixture, the mobility is lowered and a 
Table V. Range of the predicted values of the ion: mobility at 760 tor $\mathrm{r}, 298^{\circ} \mathrm{K}$. a

\begin{tabular}{|c|c|c|c|c|c|c|c|c|}
\hline \multirow[b]{2}{*}{$\begin{array}{c}\text { Neutral } \\
\text { Gas }\end{array}$} & \multirow[b]{2}{*}{. } & \multicolumn{6}{|c|}{ Ion Molecular Weight } & \multirow{2}{*}{138} \\
\hline & & 30 & 46 & 60 & 76 & 92 & 122 & \\
\hline $\mathrm{He}$ & & $\begin{array}{l}16.8 \\
18.6\end{array}$ & $\begin{array}{l}13.6 \\
18.2\end{array}$ & $\begin{array}{l}11.5 \\
17.3\end{array}$ & $\begin{array}{l}11.5 \\
17.2\end{array}$ & $\begin{array}{l}11.4 \\
17.1\end{array}$ & $\begin{array}{l}10.0 \\
16.1\end{array}$ & $\begin{array}{l}10.0 \\
16.1\end{array}$ \\
\hline Ar & & $\begin{array}{l}3.02 \\
3.07\end{array}$ & $\begin{array}{l}2.70 \\
2.75\end{array}$ & $\begin{array}{l}2.52 \\
2.60\end{array}$ & $\begin{array}{l}2.41 \\
2.48\end{array}$ & $\begin{array}{l}2.34 \\
2.40\end{array}$ & $\begin{array}{l}2.24 \\
2.32\end{array}$ & $\begin{array}{l}2.21 \\
2.28\end{array}$ \\
\hline $\mathrm{Kr}$. & & $\begin{array}{l}2.16 \\
2.19\end{array}$ & $\begin{array}{l}1.86 \\
1.90\end{array}$ & $\begin{array}{l}1.73 \\
1.75\end{array}$ & $\begin{array}{l}1.62 \\
1.64\end{array}$ & $\begin{array}{l}1.54 \\
1.56\end{array}$ & $\begin{array}{l}1.43 \\
1.47\end{array}$ & $\begin{array}{l}1.40 \\
1.43\end{array}$ \\
\hline $\mathrm{Xe}$ & & $\begin{array}{l}1.60 \\
1.62\end{array}$ & $\begin{array}{l}1.35 \\
1.38\end{array}$ & $\begin{array}{l}1.23 \\
1.26\end{array}$ & $\begin{array}{l}1.14 \\
1.17\end{array}$ & $\begin{array}{l}1.08 \\
1.10\end{array}$ & $\begin{array}{l}1 \cap 1 \\
1.03\end{array}$ & $\begin{array}{l}0.97 \\
0.99\end{array}$ \\
\hline $\mathrm{H}_{2}$ & . & $\begin{array}{l}13.3 \\
13.3\end{array}$ & $\begin{array}{l}12.7 \\
13.2\end{array}$ & $\begin{array}{l}11.8 \\
13.1\end{array}$ & $\begin{array}{l}11.8 \\
13.1\end{array}$ & $\begin{array}{l}11.8 \\
13.0\end{array}$ & $\begin{array}{l}11.0 \\
12.9\end{array}$ & $\begin{array}{l}11.0 \\
12.9\end{array}$ \\
\hline $\mathrm{D}_{2}$ & & $\begin{array}{r}9.8 \\
9.8\end{array}$ & $\begin{array}{l}9.2 \\
9.6\end{array}$ & $\begin{array}{l}8.5 \\
9.5\end{array}$ & $\begin{array}{l}8.5 \\
9.4\end{array}$ & $\begin{array}{l}8.5 \\
9.4\end{array}$ & $\begin{array}{l}7.9 \\
9.3\end{array}$ & $\begin{array}{l}7.9 \\
9.2\end{array}$ \\
\hline $\mathrm{N}_{2}$ & & $\begin{array}{l}3.18 \\
3.20\end{array}$ & $\begin{array}{l}2.89 \\
2.94\end{array}$ & $\begin{array}{l}2.71 \\
2.80\end{array}$ & $\begin{array}{l}2.62 \\
2.70\end{array}$ & $\begin{array}{l}2.55 \\
2.64\end{array}$ & $\begin{array}{l}2.40 \\
2.56\end{array}$ & $\begin{array}{l}2.37 \\
2.53\end{array}$ \\
\hline $\mathrm{N}_{2}^{\mathrm{b}}$ & & 2.92 & 2.67 & 2.51 & 2.50 & 2.39 & 2.31 & 2.27 \\
\hline
\end{tabular}

${ }^{a}$ In units of $\mathrm{cm}^{2} /(\mathrm{V}$ sec). The range in the values is caused by the range in the values of $s$ used.

b Determined by comparison with the experimental data for the mobility of alkali ions in $\mathrm{N}_{2}$ (reference 33 ). 
molecular weight of 92 or greater is indicated. When 300 to $400 \mu$ of $\mathrm{H}_{2} \mathrm{O}$ is added to the NO-Ar mixture, an ion molecular weight of more than 100 is indicated. Adding 3 or 4 torr of $\mathrm{O}_{2}$ to the NO-Ar gives variable results, in one case indicating and ion molecular weight around 92; in another, a molecular weight between 30 and 46; and in a third case, a molecular weight around 76. The addition of 200 to $400 \mu$ of $\mathrm{N}_{2} \mathrm{O}$ to the NO-Ar gaveresults ofionmolecular weights of approx 76, 30, and between 60 and 76. Finally, adding 3 to 4 torr of $\mathrm{CO}_{2}$ reduced the mobility considerably and indicated an ion molecular weight greater thail 138 .

In NO-Kr mixtures, the indicated ion molecular weight is 76 to 138, whereas it is 76 to 122 in $\mathrm{NO}-\mathrm{Xe}$ mixtures. In $\mathrm{NO}-\mathrm{H}_{2}$ mixtures the mobility is not sensitive to the ion mass, and any ion molecular weight between 30 and 138 would be consistent with the results. In the $\mathrm{NO}-\mathrm{D}_{2}$ mixtures, one determination indicates an ion molecular weight of 60 to 138 (or higher), whereas the other two determinations give ion molecular weights of 30 to 60 ; however, the uncertainty of the results do not allow us to rule out any ion molecular weight from 30 to 150 .

Mixtures of $\mathrm{NO}$ with $\mathrm{N}_{2}$ gave variable results. When compared with the mobilities as estimated from the alkali ion mobilities, the results of various runs indicate ion molecular weights of approximately $26,37,46$, and 120 . When several $\mu$ of $\mathrm{NO}_{2}$ is added to the $\mathrm{NO}-\mathrm{N}_{2}$ mixtures, ion molecular weights of around 92 and of more than 138 are indicated.

The results from the mixtures of NO with the light third-body gases $\left(\mathrm{H}_{2}, \mathrm{D}_{2}\right.$, and $\left.\mathrm{He}\right)$ give little information about the ion masses. The results in the mixtures of NO with the heavier gases indicate average ion molecular weights ranging from less than 30 to 138 . The uncertainty in the determination of $\mathrm{k}_{\mathrm{a}}$ is large enough so that most of the results in $\mathrm{NO}-\mathrm{Ar}$ and $\mathrm{NO}-\mathrm{N}_{2}$ mixtures could be interpreted as indicating average ion molecular weights of 46 or less; however, the NO-Kr and NO-Xe results definitely indicate ion molecular weights greater than 46 . 
From the results when $\mathrm{NO}_{2}$ or $\mathrm{H}_{2} \mathrm{O}$ is added, we know that ion clustering does take place when there is a sufficient quantity of molecules with large polarizabilities or with appreciable dipole moments. Thus, there may be an appreciable fraction of the ions present as ion clusters. These may be complexes with the neutral NO to give species such as $(\mathrm{NO})_{2}^{+}$or $\mathrm{NO}_{2}(\mathrm{NO})^{-}$(the smaller. size of the $\mathrm{NO}^{+}$. should enhance its chance of cluster formation in comparison with that for the $\mathrm{NO}_{2}^{-}$). Possibly some larger clusters such as (NO) ${ }_{3}^{+}$, etc., are present. Anothor likely randidate for cluster formation is the $\mathbb{N O}_{2}$ present (a micron or so) from the photolysis of the NO, since we know that the addition of 5 or $10 \mu$ of $\mathrm{NO}_{2}$ does lower the mobility. Thus, the ion clusters mày be $\mathrm{NO}\left(\mathrm{NO}_{2}\right)^{+}$and $\left(\mathrm{NO}_{2}\right)_{2}^{-}$, with possibly some larger clusters such as $\mathrm{NO}\left(\mathrm{NO}_{2}\right)_{2}^{+}$, etc. (the number uf the sc larger slusters increasing when $\mathrm{NO}_{2}$ is added). Or, the clustering may involve the third-body gas. Table XIII in Sec. VI. A gives rough estimates of the equilibrium constants, $K$, for ion-cluster formation with the different third-body gases, and these estimates indicate that xenon is the most likely to form a cluster with the ions, followed by krypton, with $\mathrm{N}_{2}$ and Ar being less likely, and the lighter gases being even less likely. So, in the NO-Xe and NO-Kr mixtures some of the ion clusters may be NO $(\mathrm{Xe})^{\prime}$ and $\mathrm{NO}(\mathrm{Kr})^{+}$, and pussibly therc aro similar slusters with the other neutrals.

In some runs, the mobility seems to decreasc as the delay time increases - - indicating that the relative proportion of for clusters io increasing during the delay time. However, these results ate very uncertain, because the larger values of $G_{C}$ at the short delay times make the $k_{a}$ determinations sensitive to the initial ion distribution. Since the observed values of a do not decrease with increasing time, either the fraction of ions present as ion clusters doesn't change markedly during the delay times, or else the recombinalion rate constant for ion clusters is either nearly the same as or larger than the rate constant for unclustered ions.

In conclusion, this method is a rather unsatisfactory way to determine the ion masses. The method gives very little information 
about the nature of the ions in the light gas mixtures, and only crude results in the heavier gases. However, these crude results indicate that (a) it is not safe to assume that ion clusters are not present, and (b) a better method of mass analysis of the ions present should give interesting results. 


\section{RESULTS OF THE EXPERIMENTAL DETERMINATION OF THE RECOMBINATION COEFFICIENT, a}

\section{A. Second-Order Process}

The ions can disappear both by recombination (a second-order process) and by diffusion (a first-order process). At small values of the ion concentration, the first-order term dominates, and since the diffusion losses increase as the mobility increases, the first-order term eventually dominates at low enough pressures.

If we can ignore the diffusiun losses, then the integrated form of the rate expression is

$$
1 / n=1 / n_{0}+a t
$$

where $\mathrm{n}$ is the ion concentration at time $\mathrm{t}, \mathrm{n}_{0}$ is the ion concentration for $t=0$, and $\cdot a$ is the recombination coefficient. A plot of $1 / n$ vs $t$ should therefore be linear, with the slope giving the value of a. How ever, Gray and Kerr have shown that the plot of $1 / \mathrm{n}$ vs $\mathrm{t}$ may appear to be linear over a short range of $1 / n$, even if the diffusion term is large. 36 Figure 12 shows an example of a plot of $1 / n$ vs $t$. Notice that the plot is linear over a fractional change in $1 / \mathrm{n}$ of "1.8. . . The other data indicate linearity uver fractional changes of 16 to 25 for high-pressure runs and of 2 to 4 at, the lowest pressures. Gray and Kerr show that linearity over a fractional change of 4 gives an apparent a that may be 8 to $30 \%$ above the true valuc, but linearity over a fractional change of 8 should give values of a that are within a few per cent of the true values. 36

In the lowest-pressure runs made with each gas, the upward curvature of the $1 / \mathrm{n}=\mathrm{vs}-\mathrm{t}$ plots indicated that diffusion was important. In these cases, the values of the slope at short delay times were taken as approximately the true value of $a$, since the ion concentrations were largest there. Also, we attempted to correct for diffusion by plotting the data according to the differential rate law when both first-and second-order terms are present: we have

$$
-d(\ln n) / d t=a n+b,
$$




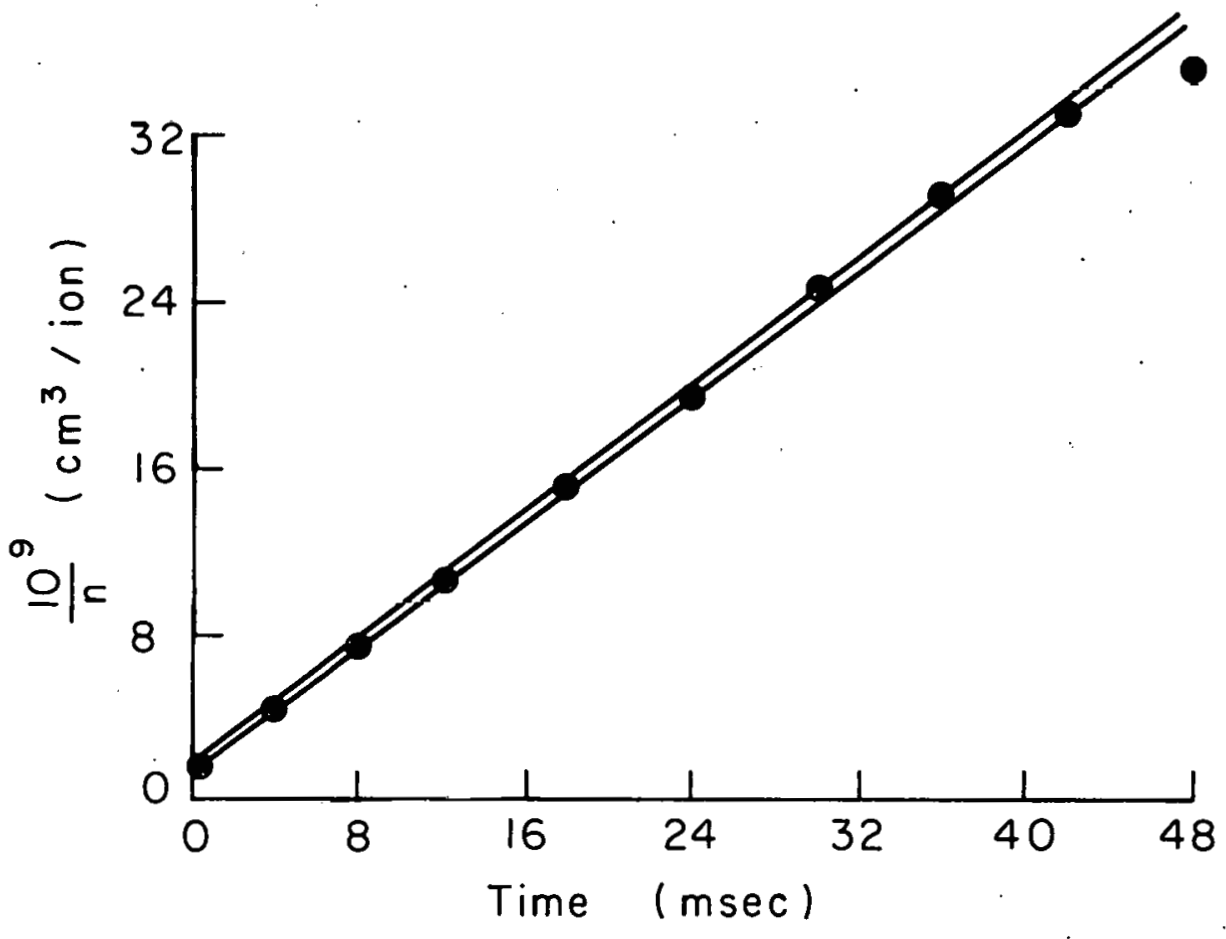

$M U-31876$

Fig. 12. Plot of $1 / \mathrm{n}$ vs time. Data are for run No. 77 in a NO-Kr mixture at a total pressure of 138 torr. The values of a from the slopes of the two lines are 7.5 and $7.6 \times 10^{-7} \mathrm{~cm}^{3} / \mathrm{sec}$. 
where $b$ is the first-order rate constant. Thus, the plot of $d \ln n / d t$ vs $n$ should give the true value of $a$. However, the $d(\ln n) / d t$ terms had to be evaluated graphically, and the uncertainty in the result was considerable.

\section{B. Initial Recombination}

The problem of initial recombination occurs when ion pairs are formed with the positive and negativeions relatively close together, so close that some of the ions recombine before a random spatial distribution is attained. This causes the observed value of a to be too large initially, with a decreasing to approach the true valuc as time increases. This difficulty was encountered by Sayels 5 and by Gardnor ${ }^{4}-$ both of whom used high pressures of electronegative gas $\left(\mathrm{O}_{2}\right)$. The problem was not encountered in these experiments because of the low pressure of electronegative gas (NO), and the observed values of a show no tendency to decrease with increasing time over periods of 50 to $75 \mathrm{msec}$.

The reasons why initial recombination should not be expected in these experiments can be understood from the following considerations. First, the photoelectron does not recombine preferentially with its parent positive ion; instead it escapes into the volume of the gas. This is because the electron is produced with 0.17 to $0.77 \mathrm{eV}$ of energy and it. has a mean free path of 3 to $20 \times 10^{-4} \mathrm{~cm}$ at a pressure of 100 tor $\mathrm{r}^{37}$ Since the Coulomb attractive energy is larger than the average thermal energy only when the charges are within $0.037 \times 10^{-4} \mathrm{~cm}$, most electrons escape to distances greater than this without a single collision. Further, the electrons are at a considerable distance away before becoming thermalized, since it takes 100 to 10,000 collisions to remove the initial kinetic energy. ${ }^{37}$ Thus we can see whether the initial spatial distribution will be inhomogeneous by considering only the distance at which. the :electron: attaches to form the negative ion.

The r.m.s. distance ${ }^{r} A$ that the electron travels before attaching can be taken approximately as 18 


$$
r_{A}=\left(6 D t_{A}\right)^{1 / 2}
$$

where $D$ is the diffusion coefficient of the electrons and $t_{A}$ is the mean time required for attachment. We can get a rough estimate of $D$ from

$$
D \approx 250\left(\frac{760}{P_{M}}\right) \mathrm{cm}^{2} / \mathrm{sec}
$$

where $P_{M I}$ is the gas pressurc in tol. ${ }^{18}$ The ${ }^{t} A$ can be estimated from $t_{A}=1 / k_{A}$, where $k_{A}$ is the first-order rate constant for electron attachment, and in Sec. II. B, $k_{A}$ is estimated to be $25+150 \mathrm{P}_{\mathrm{M}} \approx 150 \mathrm{P}_{\mathrm{M}} \mathrm{sec}^{-1}$. Substituting into Eq... (E-3) gives

$$
r_{A} \approx\left(\frac{6 \times 250 \times 760}{150\left(P_{M}\right)^{2}}\right)^{1 / 2} \approx \frac{90}{P_{M}}
$$

Thus, $r_{A}$ is on the order of 0.1 to $5 \mathrm{~cm}$--which is much larger than the average distance between ions $\left(r_{0} \approx 10^{-3} \mathrm{~cm}\right)$. We see that the negative ions do not tend to form close to the positive ions; instead, the spatial distribution of ions should be quite homogeneous even at short delay times.

C. Effects of Pressure and of Different Third-Body Gases on a

The experimental values of the specific rate of ion recombination, $a$, are listed in Table VI for various pressures of the mixtures of NO with the different $M$ gases used. The upper and lower limits of a for each pressure represent the limiting values for which reasonable straight lines could be drawn through the experimental points on the $1 / n-v s-t$ plots. There may be an error of 5 to $10 \%$ in these results as a result of uncertainty in the voltage calibration for any particular run, uncertainty in determining the areas of the current-time curves, and possible failure to keep the lamp intensity constant throughout the experiment. Also, in the runs at low pressures, there may be an additional 10 to $20 \%$ uncertainty because of the problem with the "tail" on the oscillogram. In addition there is an uncertainty of 5 to $10 \%$ in the 
Table VI. Values of a for different NO-M mixtures

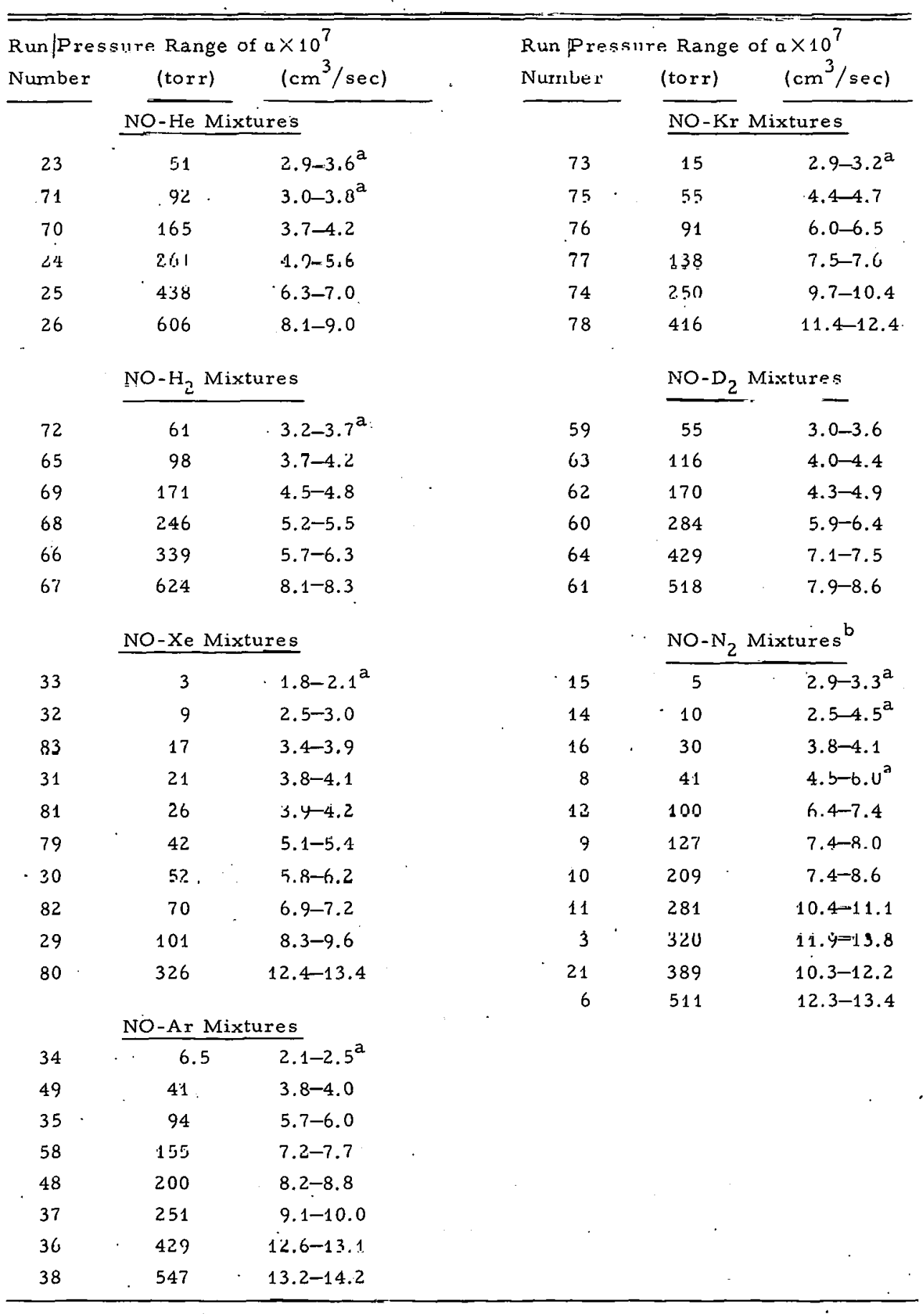

${ }^{a}$ Noticeable curvature of $1 / \mathrm{n}-\mathrm{vs}-\mathrm{t}$ plot indicates diffusion is important.

${ }^{b}$ Run No. 8 ehows diffusion berause of low value of $n_{0}$. 
absolute values of $a$ in all the experiments because of the uncertainties in determining the collection volume and in the absolute calibration of the oscilloscope. In some experiments, also, small quantities of a third gas (e.g., $\mathrm{NO}_{2}$ ) were added to the NO-M gas mixtures, and the results of these runs are given in Table VII.

The values of a plotted vs the gas pressure when $\mathrm{H}_{2}$ and when $\mathrm{Kr}$ are the third-body gases are shown in Fig. 13, Fig. 14 is for $\mathrm{D}_{2}$ and for $\mathrm{Xe}$ as the $\mathrm{M}$ gases, Fig. 15 is for $\mathrm{He}$ and for $\mathrm{N}_{2}$, and Fig. 16 gives the data when Ar is the third-body gas. The data are plotted for Loth the pure NU-M mixtures and the experiments in which other gases were added to the NO-M mixtures. Also on these figures are curves showing the predicted values of a from the results of the detailed calculation described in Sec. VII.

The general form of the a-vs-P curves has a finite value of a at zero pressure, a linear increase in a with increasing pressure at low pressure, and an approach to a constant value of a at higher pressures in the heavier gases. This general behavior is in good agreement with the results of Gardner ${ }^{4}$ and of Sayers. ${ }^{5}$ Notice that the heavier gases are more efficient third bodies, since a given pressure of a heavy gas gives a larger value of a than the same pressure of a light gas.

\section{Determination of the Low-Pressure Limit of a}

Since a extrapolates to a finite value at zero pressure and a also increases with increasing pressure, it is convenient to consider that the ion recombination mechanism consists of two parts: (a) a true bimolecular mechanism, and (b) a mechanism that is overall third order. Thus we have the reactions

$$
\mathrm{A}^{+}+\mathrm{B}^{-} \stackrel{\mathrm{k}_{0}}{\longrightarrow} \text { neutrals (2nd order), }
$$

and

$$
\mathrm{A}^{+}+\mathrm{B}^{-}+\mathrm{M} \stackrel{\mathrm{k}_{\mathrm{T}}}{\longrightarrow} \text { neutrals (overall 3rd order). (E-7) }
$$


Table VII. Effect of various added gases on a.

\begin{tabular}{|c|c|c|c|c|}
\hline \multirow[t]{2}{*}{$\begin{array}{c}\text { Run } \\
\text { number. }\end{array}$} & $\begin{array}{l}\text { Total } \\
\text { pressure } \\
\quad(\text { tor })\end{array}$ & $\begin{array}{lc}\text { added gas } \\
\text { ure } & \text { pressure } \\
\text { r) } & \text { (torr) }\end{array}$ & $\begin{array}{c}\text { NO } \\
\text { pressure } \\
\text { (torr) }\end{array}$ & $\begin{array}{c}\text { Range of } a \times 10^{7} \\
\left(\mathrm{~cm}^{3} / \mathrm{sec}\right)\end{array}$ \\
\hline & $\cdots$ & $\mathrm{NO}_{2}$ added to $\mathrm{I}$ & O-Ar mixtures & \\
\hline 39. & 32 & $\approx 0.020$ & 0.25 & $3.0-3.3$ \\
\hline \multirow[t]{2}{*}{50} & 41 & $\approx 0.005$ & 0.33 & $3.3-3.5$ \\
\hline & & \multicolumn{2}{|c|}{$\mathrm{H}_{2} \mathrm{O}$ added to NO-Ar mixtures } & \\
\hline 41 & 50 & 0.38 & 0.42 & $3.7-4.1$ \\
\hline \multirow[t]{2}{*}{42} & 48.5 & 0.28 & 0.39 & $9.6-11.1$ \\
\hline & & \multicolumn{2}{|c|}{$\mathrm{O}_{2}$ added to NO-Ar mixtures: } & \\
\hline 51 & 41 & 1.7 & 0.28 & $3.9-4.1$ \\
\hline 47 & 62 & 1.9 & 0.35 & $3.9-4.3$ \\
\hline 46 & 184 & 2.4 & 0.39 & $6.8-7.4$ \\
\hline 43 & 330 & 1.7 & 0.39 & $9.5-10.3$ \\
\hline 44 & 506 & 2.1 & 0.42 & $11.7-12.8$ \\
\hline \multirow[t]{2}{*}{45} & 507 & 4.3 & 0.35 & $12.0-13.1$ \\
\hline & & \multicolumn{2}{|c|}{$\mathrm{N}_{2} \mathrm{O}$ added to $\mathrm{NO}-\mathrm{Ar}$ mixtures } & \\
\hline 52 & 39 & 0.63 & 0.30 & $3.7-4.1$ \\
\hline 54 & 281 & 0.38 & 0.28 & $9.4-10.4$ \\
\hline \multirow[t]{2}{*}{5.5} & 509 & 0.25 & 0.34 & $11.9-12.5$ \\
\hline & & \multicolumn{2}{|c|}{$\mathrm{CO}_{2}$ added to $\mathrm{NO}-\mathrm{Ar}$ mixtures } & \\
\hline \multirow[t]{2}{*}{53} & 42 & 3.5 & 0.29 & $3.8-3.9$ \\
\hline & & \multicolumn{2}{|c|}{$\mathrm{NO}_{2}$ added to $\mathrm{NO}-\mathrm{N}_{2}$ mixtures } & \\
\hline 18 & 20 & $\approx 0.020$ & 0.26 & $3.1-3.2$ \\
\hline 17 & 52 & $\approx 0.025$ & 0.28 & $3.8-4.0$ \\
\hline 20 & 357 & $\approx 0.010$ & 0.24 & $8.7-9.6$ \\
\hline \multirow[t]{2}{*}{22} & 513 & $\approx 0.010$ & 0.17 & $12.0-12.4$ \\
\hline & & \multicolumn{2}{|c|}{$\mathrm{NO}_{2}$ added to $\mathrm{NO}-\mathrm{He}$ mixtures } & \\
\hline 27 & 175 & $\approx 0.007$ & 0.15 & $3.8-4.0$ \\
\hline
\end{tabular}




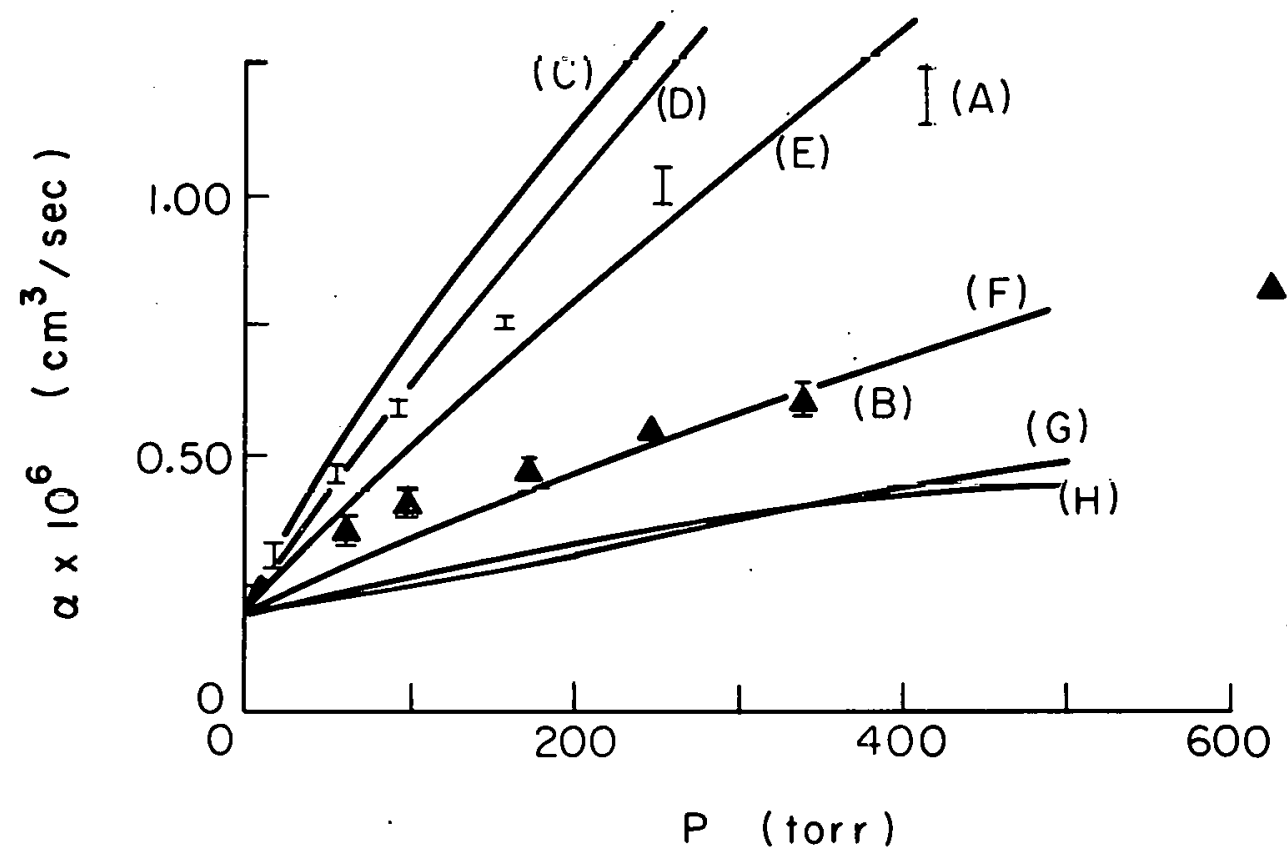

$M U .31877$

Fig. 13. Plot of a vs pressure for $\mathrm{NO}-\mathrm{Kr}$ and $\mathrm{NO}-\mathrm{H}_{2}$ mixtures: (A) data for $\mathrm{NO}-\mathrm{Kr}$ mixtures; (B) triangles are data for $\mathrm{NO}-\mathrm{H}_{2}$ mixtures; (C), (D), and (E) are predicted curves for deactivation by krypton atoms using the detailed calculation, and (F), (G), and (H) are predicted for deactivation by $\mathrm{H}_{2}$. (C), (F), (E), and (H) are for $E_{D}=0$ and $b_{m}=600 \AA$. (C) and $(F)$ are for $\mathrm{NO}^{+}$and $\mathrm{NO}_{2}^{-}$ ions. (E) and $(\mathrm{H})$ are for $\mathrm{NO}\left(\mathrm{NO}_{2}\right)^{+}$and $\left(\mathrm{NO}_{2}\right)_{2}^{-}$ions. (D) and (G) are for $E_{D}=k_{B} T, b_{m} \geqslant 1500^{2} \AA$, and $N^{+}$and $N_{2} \bar{i}$ ions. 


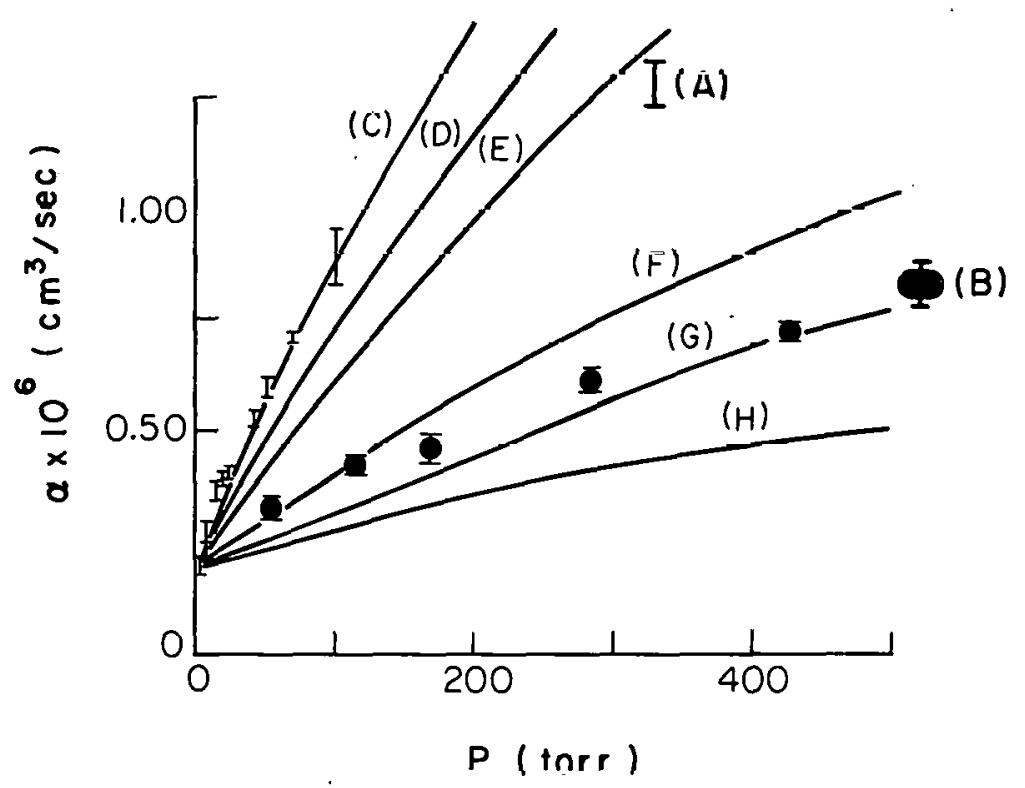

$M U-31878$

Fig. 14. Plot of a vs pressure for $\mathrm{NO}-\mathrm{Xe}$ and $\mathrm{NO}-\mathrm{D}_{2}$ mixtures: (A) data for NO-Xe mixtures; (B) circles are data for $\mathrm{NO}-\mathrm{D}_{2}$ mixtures; (C), (D), and (E) are predicted curves for deactivation by xenon atoms using the detailed calculation, and $(F)$, $(G)$, and $(H)$ are predicted for deactivation by $D_{2} \cdot(C),(F)$, $(E)$, and $(H)$ are for $E_{D}=0$ and $b_{m}=600 \AA \quad(C)$ and $(F)$ are for NOt and $\mathrm{NO}_{2}^{-}$ions. $(\mathrm{E})$ and $(\mathrm{H})$ are for $\mathrm{NO}\left(\mathrm{NO}_{2}\right)^{+}$, and $\left(\mathrm{NO}_{2}\right)_{\overline{2}}$ ions. (D) and $(\mathrm{G})$ are for $\mathrm{E}_{\mathrm{D}}=\mathrm{k}_{\mathrm{B}} \mathrm{T}, \mathrm{b}_{\mathrm{m}} \geqslant 1500 \AA$, and $\mathrm{NO}^{+}$and $\mathrm{NO}_{2}^{-}$ions. 


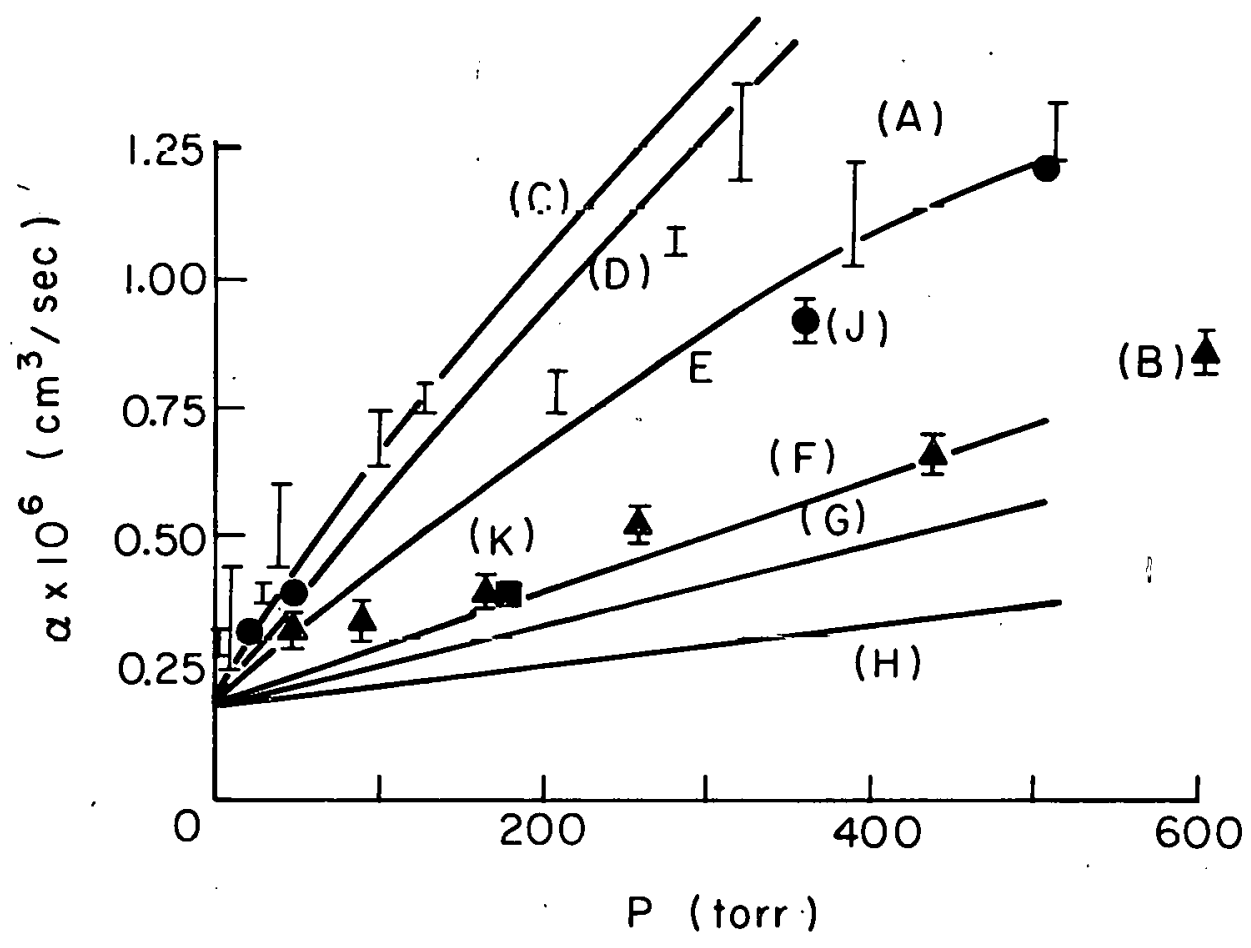

MU.31879

Fig. 15. Plot of a vs pressure for $\mathrm{NO}-\mathrm{N}_{2}, \mathrm{NO}-\mathrm{He}, \mathrm{NO}-\mathrm{NO}_{2}-\mathrm{N}_{2}$, and $\mathrm{NO}-\mathrm{NO}_{2}-\mathrm{He}$ mixtures: (A) data for $\mathrm{NO}-\mathrm{N}_{2}$ mixtures; (B) triangles are data for $\mathrm{NO}-\mathrm{He}$ mixtures; (C), (D), and (E) are predicted curves for deactivation by $\mathrm{N}_{2}$, and $(F),(G)$, and (H) a re predicted for deactivation by $\mathrm{He}$. (C), (F), (E), and $(\mathrm{H})$ are for $E_{D}=0$ and $b_{m}=600 \AA$. (C) and $(F)$ are for $\mathrm{NO}^{+}$and $\mathrm{NO}_{2}{ }^{-}$ions. (E) and $(\mathrm{H})$ are for $\mathrm{NO}\left(\mathrm{NO}_{2}\right)^{+}$and $\left(\mathrm{NO}_{2}\right) 2^{-}$ions. (D) and $(G)$ are for $E_{D}=k_{B} T, b_{m} \geqslant 1500 \AA$, and $\mathrm{NO}^{+}$and $\mathrm{NO}_{2}^{-}$ions. (J) circles are data for $\mathrm{NO}-\mathrm{NO}_{2}-\mathrm{N}_{2}$ mixtures. (K) square is the datum for the $\mathrm{NO}-\mathrm{NO}_{2}-\mathrm{He}$ mixture. 


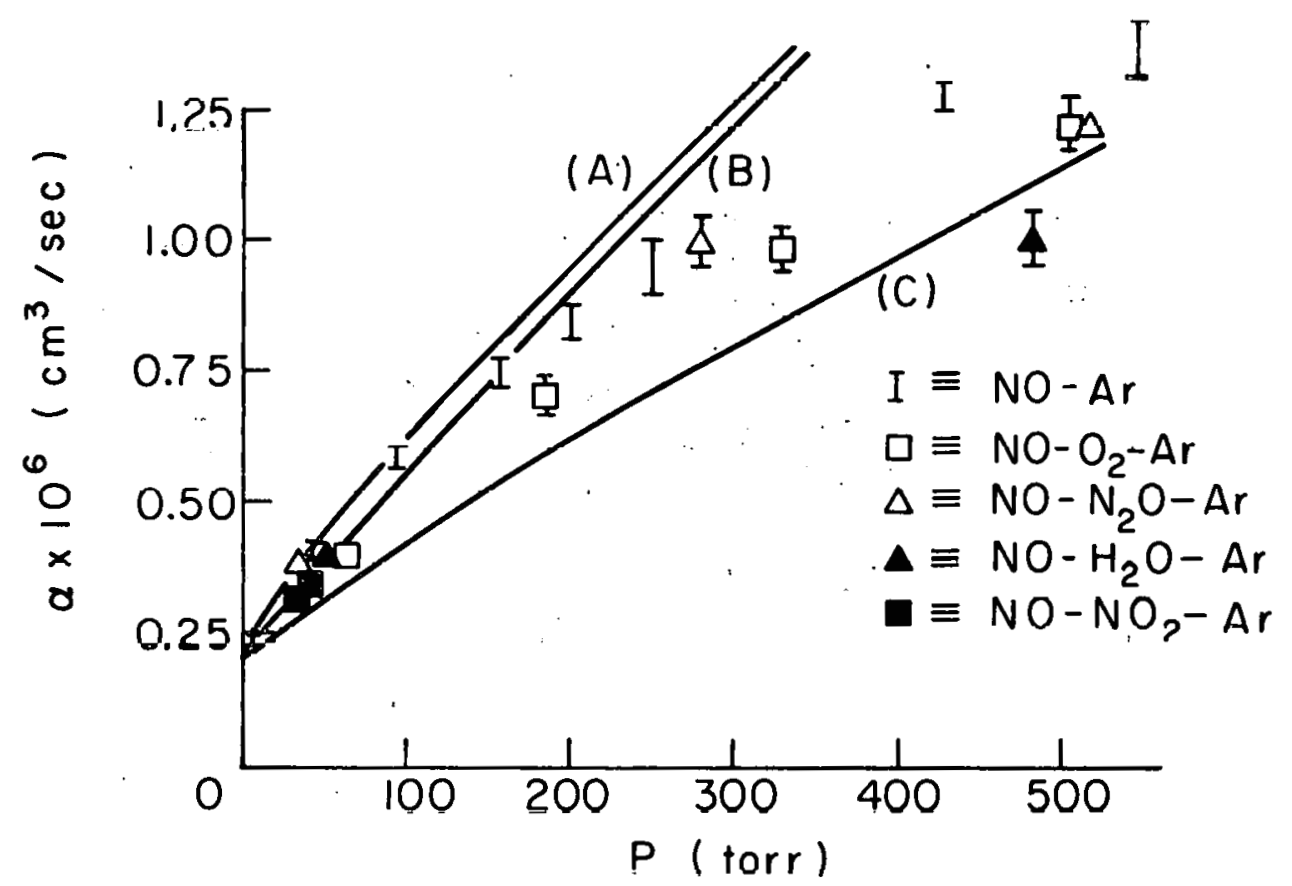

$M U-31880$

Fig. 16. Plot of a vs pressure for $\mathrm{NO}-\mathrm{Ar}$, NO-O $2-\mathrm{Ar}, \mathrm{NO}-\mathrm{N}_{2} \mathrm{O}-\mathrm{Ar}$, $\mathrm{NO}-\mathrm{H}_{2} \mathrm{O}-\mathrm{Ar}$, and $\mathrm{NO}-\mathrm{NO}_{2}-\mathrm{Ar}$ mixtures; (A) predicted curve for $E_{D}=O, b_{m}=600 \AA$, and $N^{+}$and $N_{2}^{-}$ions; (B) predicted for $E_{D}=k_{B} T, b_{m} \geqslant 1500 \AA$, and $N^{+}$and $N_{2}^{2}$ ions; (C) predicted for $\mathrm{E}_{\mathrm{D}}=0, \mathrm{~b}_{\mathrm{m}}=600 \AA$, and $\mathrm{NO}\left(\mathrm{NO}_{2}\right)^{\prime}$ and $\left(\mathrm{NO}_{2}\right)_{2}$ ions. 
The total rate of recombination is the sum of the rates of Reactions $(E-6)$ and $(E-.7)$, and it is given by

$$
\frac{-d n}{d t}=\left(k_{0}+k_{T}[M]\right) n^{2}=a n^{2},
$$

where $n=\left[\mathrm{A}^{+}\right]=\left[\mathrm{B}^{-}\right]$. Therefore we have

$$
a \mp k_{0}+k_{T}[M]
$$

where $k_{0}$ is the rate constant for the bimolecular process and is the limit of $a$ at zero pressure, and $k_{T}$ is the termolecular rate constant, which depends upon the nature of the third-body gas.

Table VIII gives the values of $k_{0}$ determined by a linear extrapolation of the low-pressure values of a to the zero pressure limit. Also included are the upper and lower limits of the values of $k_{0}$ that could be consistent with the extrapolation in each of the third-body gases. Within the experimental scatter, the values of $k_{0}$ are the same for all the third-bidy gases. This must be the result if $k_{0}$ is for a truly bimolecular process between only the positive and negative ions, and if the ions are the same in each gas mixture. Unfortunately, the uncertainty is so large that a change of $40 \%$ in $\mathrm{k}_{0}$ would be required in $\mathrm{k}_{0}$ before it would be evident. The average value of $\mathrm{k}_{0}$ is $\mathrm{k}_{0}=2.1 \pm 0.4 \times 10^{-7} \mathrm{~cm}^{3} / \mathrm{sec}$.

Since $k_{0}=\sigma_{0}\langle g\rangle$, where $\sigma_{0}$ is the cross section and $\langle g\rangle$ is the mean relative velocity, we can evaluate $\sigma_{0}$ if we know the mass of the ions (which determines $\langle g\rangle$ ). If we assume the ions to be $\mathrm{NO}^{+}$ and $\mathrm{NO}_{2}^{-}$, then $\sigma_{0}=3.6 \pm 0.7 \times 10^{-12} \mathrm{~cm}^{2}$. If we assume that the ions have a gram-molecular weight of 76, then $\sigma_{0}=5.2 \pm 1.0 \times 10^{-12} \mathrm{~cm}^{2}$. These experimental values may be compared with Yeung's value of $k_{0}=1.47 \times 10^{-7} \mathrm{~cm}^{3} / \mathrm{sec}$ for ions in iodine vapor; ${ }^{2,6}$ if the ions are $\mathrm{I}_{2}^{+}$and $\mathrm{I}^{-}$, then $\sigma_{0}=5.4 \times 10^{-12} \mathrm{~cm}^{2}$. Also, for ions in bromine vapor, Yeung obtained $\mathrm{k}_{0}=1.85 \times 10^{-7} \mathrm{~cm}^{3} / \mathrm{sec}$, which corresponds to $\sigma_{0}=5.4 \times 10^{-12} \mathrm{~cm}^{2}$ if the ions are $\mathrm{Br}_{2}^{+}$and $\mathrm{Br}^{-}$. Greaves found values of $\mathrm{k}_{0}=10^{-7} \mathrm{~cm}^{3} / \mathrm{sec}$ and $\sigma_{0}=3 \times 10^{-12^{2}} \mathrm{~cm}^{2}$ for $\mathrm{I}_{2}^{+}$and $\mathrm{I}^{-} .^{2}$ Therefore the cross section for the bimolecular process determined in these 
experiments is quite comparable to the results for ions in iodine vapor and in bromine vapor.

Table VIII. Low-pressure limit of $a$ in various NO-M mixtures

\begin{tabular}{ccc}
\hline gas & $\begin{array}{c}\mathrm{k}_{0} \times 10^{7} \\
\left(\mathrm{~cm}^{3} / \mathrm{sec}\right)\end{array}$ & $\begin{array}{c}\text { Range of } \mathrm{k}_{0} \times 10^{7} \\
\left(\mathrm{~cm}^{3} / \mathrm{sec}\right)\end{array}$ \\
\hline He & 1.9 & $1.5-2.5$ \\
$\mathrm{Ar}$ & 2.1 & $1.7-2.3$ \\
$\mathrm{Kr}$ & 2.3 & $2.1-2.5$ \\
$\mathrm{Xe}$ & 1.8 & $1.6-2.4$ \\
$\mathrm{H}_{2}$ & 2.4 & $2.1-2.6$ \\
$\mathrm{D}_{2}$ & 2.1 & $1.7-2.7$ \\
$\mathrm{~N}_{2}$ & 2.2 & $1.7-2.5$ \\
\hline
\end{tabular}

E. Termoleculàr Charge Neutralization

We now discuss the termolecular charge neutralization reaction, whose overall reaction is given by Eq. (E-7).

\section{Mechanismr}

The overall third-order charge-neutralization reaction can be described in terms of the following collisional-deactivation mechanism. An excited ion pair, $\left(\mathrm{A}^{+} \mathrm{B}^{-}\right)^{*}$, is formed by

$$
A^{+}+B^{-} \stackrel{\dot{k}_{1}}{\longrightarrow}\left(A^{+} B^{-}\right)^{*} \text {. }
$$

Then it can either dissociate,

$$
\left(\mathrm{A}^{+} \mathrm{B}^{-}\right)^{*} \stackrel{\mathrm{k}_{2}}{\longrightarrow} \mathrm{A}^{+}+\mathrm{B}^{-}
$$

or can be deactivated by a collision with a neutral gas molecule,

$$
\left(\mathrm{A}^{+} \mathrm{B}^{-}\right)^{*}+\mathrm{M} \stackrel{\mathrm{k}_{3}}{\rightarrow}\left(\mathrm{A}^{+} \mathrm{B}^{-}\right)+\mathrm{M} \text {. }
$$


The deactivated ion pair, $\left(\mathrm{A}^{+} \mathrm{B}^{-}\right)$, may be considered to be an ion pair that has lost enough of its kinetic energy of relative motion so that the ions can no longer separate to large distances (i.e., dissociate). Since the $\left(\mathrm{A}^{+} \mathrm{B}^{-}\right)$cannot dissociate, the ions oscillate between their minimum and maximum separations until charge neutralization eventually occurs.

The excited ion pair, $\left(\mathrm{A}^{+} \mathrm{B}^{-}\right)^{*}$, may be thought of as being two ions close enough together so that a collision with a neutral can deactivate the ion pair. Also, since we want to consider here only the termolecular charge-neutralization process, we do not include as $\left(\mathrm{A}^{+} \mathrm{B}^{-}\right)^{*}$ any ion pairs that are in the process of undergoing the purely birnolecular charge-neutralization reaction. That is, we do not include ion pairs whose impact parameters are less than $b_{0}=\left[k_{0} /(\pi\langle g\rangle)\right]^{1 / 2}$, where $k_{0}$ is the bimolecular rate constant determined in Sec. V. D and $\langle\mathrm{g}\rangle$ is the mean relative velocity of the ions. We can express $\mathrm{k}_{1}$ as

$$
k_{1}=\pi\left(b_{1}^{2}=b_{0}^{2}\right)\langle g\rangle \text {. }
$$

Therefore the ion pairs with impact parameters between $b_{0}$ and $b_{1}$ are to be included as $\left(\mathrm{A}^{+} \mathrm{B}^{-}\right)^{*}$.

When the pressure is more than 1 to 2 atm, the value of $k_{1}$ begins to decrease noticeably as the pressure increases, and even at pressures below 1 atmosphere, $k_{1}$ depends somewhat on the pressure. However, the decrease in $k_{1}$ should not be more than 10 to $15 \%$ in the pressure range used, and we consider $k_{1}$ to be independent of the pressure.

If we assume that every deactivated ion pair eventually undergoes charge neutralization, and if we use the steady-state assumption for $\left[\left(A^{+} B^{-}\right)^{*}\right]$, then from Reactions $(E-7),(E-10),(E-11)$, and $(E-12)$, the expression for $a_{T}$, the termolecular part of the recombination coeffiçient, is

$$
a_{T}=k_{T}[M]=a-k_{0}=\frac{k_{1} k_{3}[M]}{k_{2}+k_{3}[M]} \text {, }
$$

where $[M]$ is the neutral concentration. 


\section{Experimental Determination of $\mathrm{k}_{1}$}

In order to evaluate the rate constants for the individual steps in the mechanism, it is convenient to invert Eq. $(E-14)$ to give

$$
1 / a_{T}=1 /\left(a-k_{0}\right)=1 / k_{1}+k_{2} / k_{1} k_{3}(1 /[M]) .
$$

A plot of $1 / a_{\mathrm{T}}$ vs $1 /[\mathrm{M}]$ (or vs $1 /$ pressure) should therefore be linear, with an intercept of $1 / k_{1}$ and a slope of $k_{2} /\left(k_{1} k_{3}\right)$. Figure 17 shows a plot of $1 / \mathrm{a}_{\mathrm{T}}$ vs the reciprocal of the pressure fus lie data in the $\mathrm{NU}-\mathrm{H}_{2}$ NO-Ar, and NO-Xe mixtures, and Fig. 18 shows the data for the NO-He and NO-N $\mathrm{N}_{2}$ mixtures. Figure 19 shows the data for the $\mathrm{NO}-\mathrm{D}_{2}$-and $\mathrm{N} \Omega-\mathrm{Kr}$ mixtures, along with the lines deterrnined by the data shown in Figs. 17 and 18. Table IX gives the values of $k_{1}$ determined in each $M$ gas, and also gives the upper and lower limits of the values of $k_{1}$ that would seem to be consistent with the data. The values of $k_{1}$ show a rather wide variation, and it may be $k_{1}$ is not independent of the $M$ gas. However, because of the large experimental uncertainty, we decided to assume that $k_{1}$ is independent of the $M$ gas. The average value of $k_{1}$ is $k_{1}=1.8 \pm 0.5 \times 10^{-6} \mathrm{~cm}^{3} / \mathrm{sec}$. Therefore, the highpressure limit of the specific rate of recombination is $a=k_{1}+k_{0}=2.0 \pm 0.5 \times 10^{-6} \mathrm{~cm}^{3} / \mathrm{sec}$.

By knowing $k_{0}$, and $k_{1}$, we can calculate the impact parameter $\mathrm{b}_{1}$ from $\mathrm{E}_{\mathrm{f}}(\mathrm{F},-13)$, if we know the ion masses. Also, we can calculate the distance of closest approach, ${ }^{r} C$, corresponding to $b_{1}$. If we assume that the ions are $\mathrm{NO}^{+}$and $\mathrm{NO}_{2}^{-}$, then the cross section, $\sigma_{1}$, is $\sigma_{1}=\pi \mathrm{b}_{1}^{2}=3.4 \pm 0.9 \times 10^{-11} \mathrm{~cm}^{2} ; b_{1}=3.3 \pm 0.4 \times 10^{-6} \mathrm{~cm}$; and ${ }^{\mathrm{C}}=1.8 \pm 0.4 \times 10^{-6} \mathrm{~cm}$. The Coulomb energy at $\mathrm{r}_{\mathrm{C}}$ is $3.2 \pm 0.8 \mathrm{k}_{\mathrm{B}} \mathrm{T}$. Since the ions may be clustered, we have also calculated the values assuming that both ions have a gram molecular weight of $76 .{ }^{\circ}$ Then $\sigma_{1}=4.9 \pm 1.2 \times 10^{-11} \mathrm{~cm}^{2} ; b_{1}=4.0 \pm 0.6 \times 10^{-6} \mathrm{~cm} ; r_{C}=2.3 \pm 0.4 \times 10^{-6} \mathrm{~cm}$; and the Coulomb energy at $r_{C}$ is $2.4 \pm 0.6 k_{B} T$. 


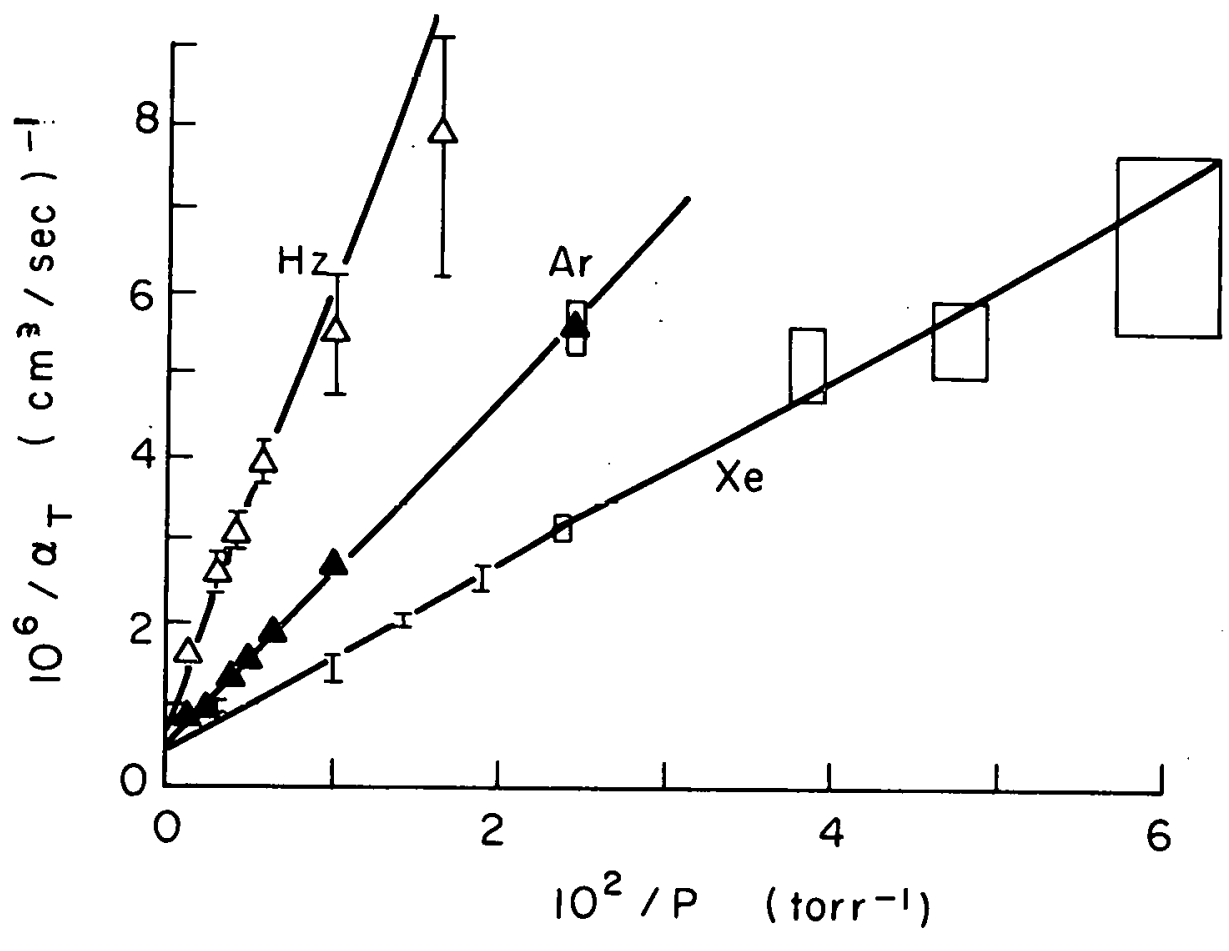

$M U .31881$

Fig. 17. Plol of $1 / a_{T}$ vs $1 / \mathrm{P}$ for $\mathrm{NO}-\mathrm{H}_{2}$, NO-Ar and NO-Xe mixtures. Open triangles are data in $\mathrm{NO}-\mathrm{H}_{2}$; solid triangles are data in NO-Ar; and the other points are data in $\mathrm{NO}-\mathrm{Xe}$. 


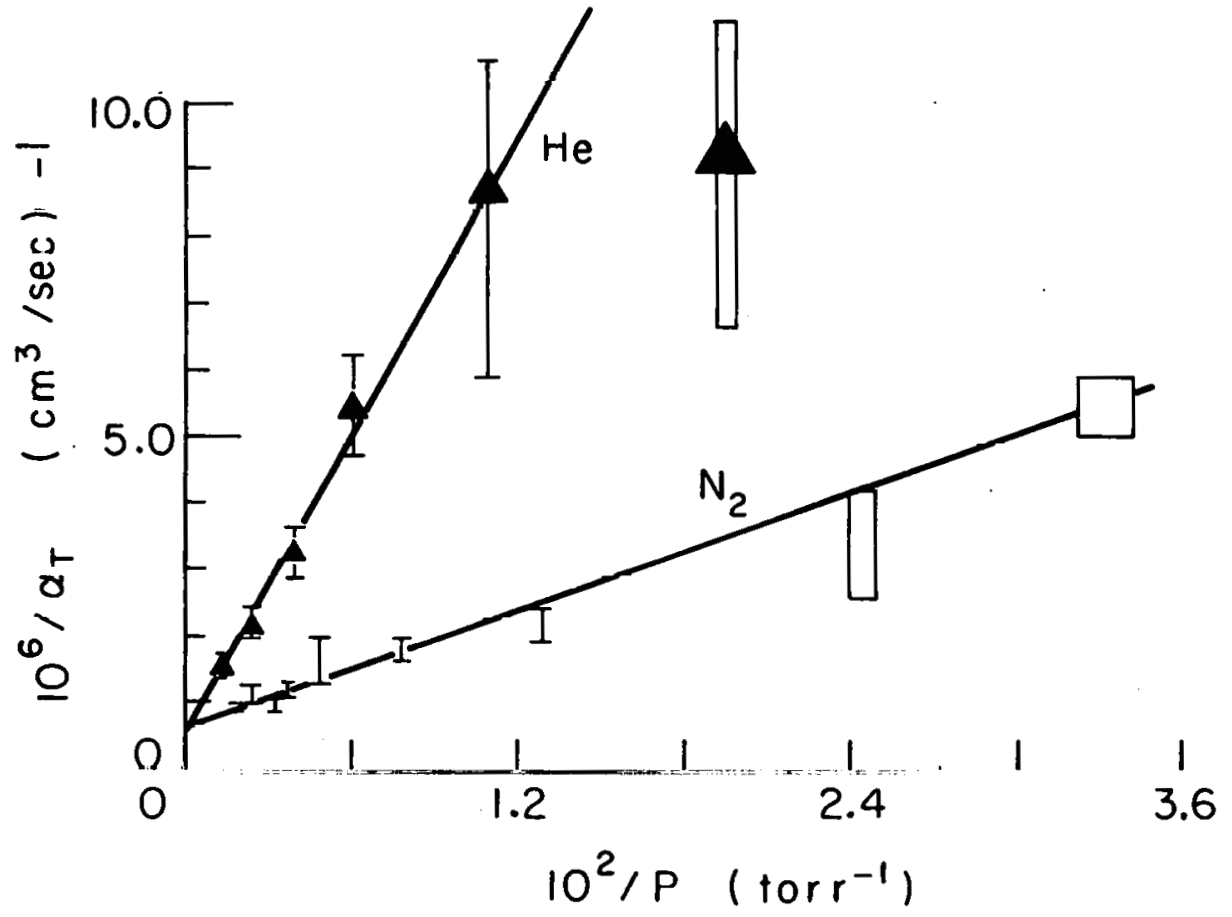

$M U \cdot 31882$

Fig. 18. Plot of $1 / \mathrm{a}_{\mathrm{T}}$ vs $1 / \mathrm{P}$ for $\mathrm{NO}-\mathrm{He}$ and $\mathrm{NO}-\mathrm{N}_{2}$ nixtures. Triangles are data in NO-He and the other points are data in $\mathrm{NO}-\mathrm{N}_{2}$. 


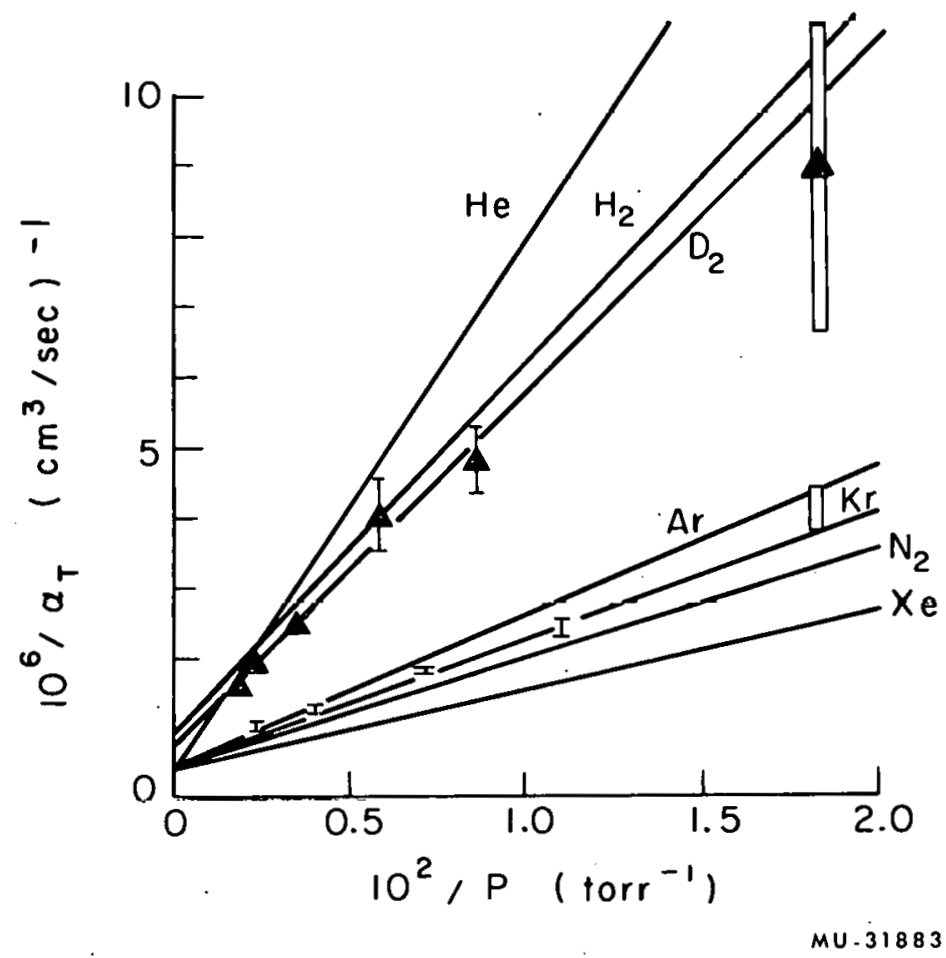

Fig. 19. Plot of $1 / a_{T}$ vs $1 / P$ for $N O-D_{2}$ and $\mathrm{NO}-\mathrm{Kr}$ mixtures along with the best lines from the other gas mixtures. Triangles are data in $\mathrm{NO}-\mathrm{D}_{2}$ and the other points are for NO-Kr mixtures. 
Table IX. Values of $k_{1}$ in various NO-M mixtures.

\begin{tabular}{lcc}
\hline gas & $\begin{array}{c}\mathrm{k}_{1} \times 10^{6} \\
\left(\mathrm{~cm}^{3} / \mathrm{sec}\right)\end{array}$ & $\begin{array}{c}\text { Range of } \mathrm{k}_{1} \times 10^{6} \\
\left(\mathrm{~cm}^{3} / \mathrm{sec}^{6}\right)\end{array}$ \\
\cline { 2 - 2 } & 2.4 & $1.4-3.6$ \\
$\mathrm{Hr}$ & 2.2 & $1.8-2.6$ \\
$\mathrm{Kr}$ & 1.8 & $1.6-2.0$ \\
$\mathrm{Xe}$ & 2.1 & $1.9-2.6$ \\
$\mathrm{H}_{2}$ & 1.1 & $0.9-1.4$ \\
$\mathrm{D}_{2}$ & 1.3 & $1.1-1.8$ \\
$\mathrm{~N}_{2}$ & 1.6 & $1.5-1.8$ \\
\hline \hline
\end{tabular}

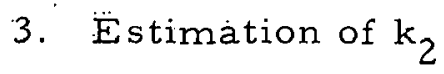

The value of the rate constant for dissociation of the $\left(\mathrm{A}^{+} \mathrm{B}^{-}\right)^{*}$ can be estimated by noting $\mathrm{k}_{2}=1 / \tau$, where $\tau$ is the lifetime of the $\left(\mathrm{A}^{+} \mathrm{B}^{-}\right)^{*}$ if no deactivation occurs. We have considered that the $\left(\mathrm{A}^{+} \mathrm{B}^{-}\right)^{*}$ state is defined as ion pairs within the distance of closest approach corresponding to $b_{1}$. Then we assume that when the ions are separated by a long distance their relative velocity has the mean value $\left(=\left[8 \mathrm{k}_{\mathrm{B}} \mathrm{T} /(\mu \pi)\right]^{1 / 2}\right)$, so that we can now calculate $\tau$ from the equations of the hyperbolic orbit for each value of $b$. What we need is the average of the reciprocal of $\tau$ for ion pairs with impact parameters between $b_{0}$ and $b_{1}$. In the detailed calculation presented in Section VII. $D$, the same type of average is required, and the details of how the calculation is done are given there. The results of the calculation are that if the ions are $\mathrm{NO}^{+}$and $\mathrm{NO}_{2}^{-}$, with $\mathrm{k}_{0}=2.1 \times 10^{-7} \mathrm{~cm}^{3} / \mathrm{sec}$ and $\mathrm{k}_{1}=1.8 \pm 0.5 \times 10^{-6}$ $\mathrm{cm}^{3} / \mathrm{sec}$, then $\mathrm{k}_{2}=4.5_{-0.9}^{+1.6} \times 10^{10} \mathrm{sec}^{-1}$. If the ions have a gram molecular weight of 76 , then $\mathrm{k}_{2}=2.2_{-0.1}^{+0.7} \times 10^{10} \mathrm{sec}^{-1}$.

The $k_{2} / k_{1}$ ratio is approximately proportional to the reciprocal of the cube of $r_{C}$ corresponding to $b_{1}$, so it is :roughly proportional to $1 / b_{1}^{3}$. Thüs, if $k_{1}$ is independent of the $M$ gas, then $k_{2}$ is also 
independent. However, if $k_{1}$ is not independent of $M$, then the ratios of $k_{2}$ for different $M$ gases are approximately inversely proportional to the ratios of $k_{1}^{1 / 2}$ for the different $M$ gases.

4. Determination of the Relative Third-Body Efficiencies:

and the Estimation of $\mathrm{k}_{3}$

If we assume $k_{1}$ to be independent of the $M$ gas, then the ratios of the reciprocals of the slopes of the plots of $1 / a_{T}$ vs $1 /[\mathrm{M}]$ (the slope is $\left.k_{2} /\left(k_{1} k_{3}\right)\right)$ give the ratios of $k_{3}$, the rate constant for the deactivation of $\left(\mathrm{A}^{+} \mathrm{B}^{-}\right)^{* *}$. Table $\mathrm{X}$ gives the values of $\mathrm{k}_{2} /\left(\mathrm{k}_{1} \mathrm{k}_{3}\right)$ and the relative third-body efficiencies. The data permit the relative efficiencies to be determined only within fairly wide limits, and the values are also subject to the assumption that $k_{1}$ is independent of the $M$ gas.

If we take the value of $\mathrm{k}_{1}=1.8 \times 10^{-6} \mathrm{~cm}^{3} / \mathrm{sec}$ and use the estimates that $\mathrm{k}_{2}=4.5 \times 10^{10} \mathrm{sec}^{-1}$ or: $\mathrm{k}_{2}=2.2 \times 10^{10} \mathrm{sec}^{-1}$, then we can get rough estimates of the values of $k_{3}$ for each gas. These estimates are also given in 'lable $X$. However, these values of $k_{3}$ are subject to the error in estimating $k_{2}$, as well as the uncertainty in determining $k_{1}$ and $k_{2} /\left(k_{1} k_{3}\right)$. Thus, although the values of $k_{3}$ are rather uncertain and the relative efficiencies are determined only within limits, we see that the $M$ gases can be divided into three groups, with Xe being the most efficient third-body gas, $\mathrm{N}_{2}, \mathrm{Kr}$, and $\mathrm{Ar}$ in the middle group, and $\mathrm{D}_{2}, \mathrm{H}_{2}$, and $\mathrm{He}$ in the least efficient group. 
Table X. Relative third-body efficiencies and estimated values of $\mathrm{k}_{3}$.

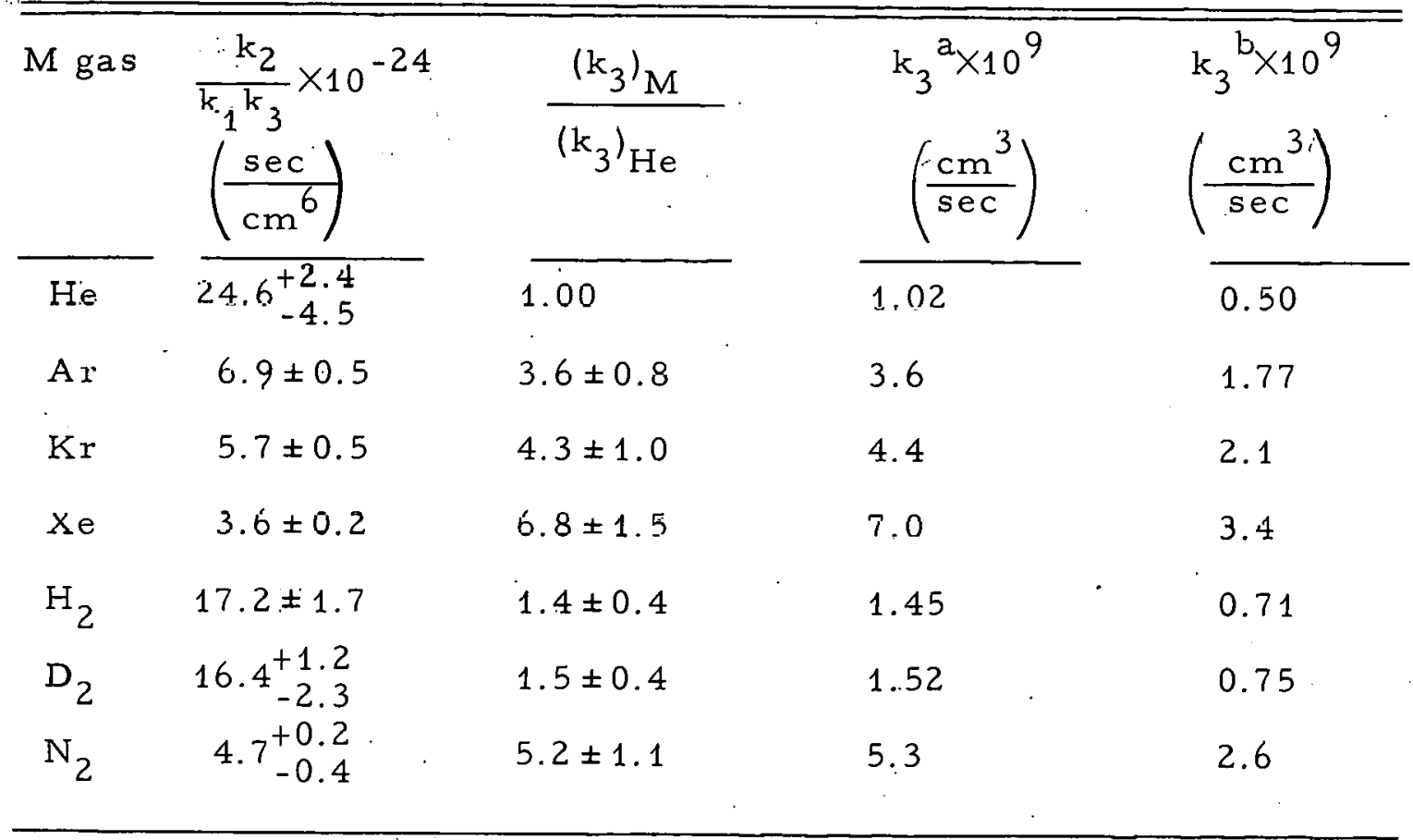

a Calculated by assuming $\mathrm{k}_{1}=1.8 \times 10^{-6} \mathrm{~cm}^{3} / \mathrm{sec}$ and $\mathrm{k}_{2}=4.5 \times 10^{10}$ $\sec ^{-1}$, which was the estimated value of $\mathrm{k}_{2}$ when $\mathrm{NO}^{+}, \mathrm{NO}_{2}^{-}$were the ions.

${ }^{b}$ Calculated by assuming $k_{1}=1.8 \times 10^{-6} \mathrm{~cm}^{3} / \mathrm{sec}$ and $\mathrm{k}_{2}=2.2 \times 10^{10} \mathrm{sec}^{-1}$, which was the estimated value of $k_{2}$ for ions having gram molecular weights of 16 . 


\section{COMPARISON OF RESUULTS WITH EXISTING THEORIES}

In this section we discuss our experimental results in terms of the Fueno-Eyring-Ree theory ${ }^{10}$ and in terms of the Natanson theory, ${ }^{9}$ which is an extension and modification of the Thomson theory. ${ }^{8}$

A. Comparison of Results with the Theory of Fuero, Eyring, and Ree Fueno, Eyring, and Ree (FER) ${ }^{10}$ presented a theory for threebody ion-ion recombination in which the mechanism is assumed to be

formation of a loose complex,

$\mathrm{M}+\mathrm{A}^{+} \underset{\mathrm{k}_{2}}{\stackrel{\mathrm{k}_{1}}{\rightarrow}} \mathrm{MA}^{+}$,

followed by an exchange reaction,

$$
\mathrm{MA}^{+}+\mathrm{B}^{-} \stackrel{\mathrm{k}_{3}}{\rightarrow} \mathrm{AB}+\mathrm{M} \text {. }
$$

For $k_{3}\left[\mathrm{~B}^{-}\right] / \mathrm{k}_{2}<<1$, the $\mathrm{MA}^{+}$is in equilibrium with the $\mathrm{A}^{+}$and the $\mathrm{M}$, undisturbed by Reaction $(F-2)$. Also if $:\left[\mathrm{MA}^{+}\right] /[\mathrm{M}]$ is small, then the overall three-body rate constant, $k_{T}$, can be represented as

$$
\mathrm{k}_{\mathrm{T}}=\mathrm{k}_{3} \mathrm{~K}
$$

where $K=k_{1} / k_{2}$ is the equilibrium constant for Eq. $(F-1)$. This mechanism is the ion-recombination analog of a mechanism for atom recombination proposed by Bunker and Davidson. ${ }^{11}$

Under the rigid rotator and harmonic oscillator assumptions, and assuming that neither of the rotational and vibrational energies can exceed the binding energy, $\epsilon_{m}$, FER arrive at the following expression for the equilibrium constant:

$$
K=\frac{h^{3}}{\left(2 \pi \mu k_{B} T\right)^{3 / 2}} \cdot \frac{8 \pi^{2} \mu r_{m}{ }^{2} k_{B} T}{h^{2}} \cdot \frac{\left[1-\exp \left(-\epsilon_{m} / k_{B} T\right)\right]^{2}}{\left[1-\exp \left(-\epsilon_{v} / k_{B} T\right)\right]} \cdot \exp \left(\epsilon_{m} / k_{B} T\right),
$$

where $\mu$ is the reduced mass of the $\mathrm{MA}^{+}$complex, ${ }^{{ }}{ }_{\mathrm{m}}$ is the equilibrium internuclear distance between $M$ and $A^{+}, \epsilon_{m}$ is the binding 
energy of the complex, and $\epsilon_{\mathrm{v}}$ is the vibrational quantum of the $\mathrm{M}-\mathrm{A}^{+}$ bond. FER show how to evaluate $\mathrm{r}_{\mathrm{m}}, \epsilon_{\mathrm{m}}$, and $\epsilon_{\mathrm{v}}$ under the assump tion that the $\mathrm{M}-\mathrm{A}^{+}$interaction potential, $V(r)$, is given by the sum of the Lennard-Jones potential and the polarization energy:

$$
V(r)=4 \quad \epsilon_{0}\left\{\left(\frac{\sigma}{r}\right)^{12}-\left(\frac{\sigma}{r}\right)^{6}\right\}-\frac{a_{M^{\Theta}}{ }^{2}}{2 r^{4}} \text {, }
$$

where $\sigma$ and $\epsilon_{0}$ are the Lcnnard-Jones parameters for the interaction between. $A$ and $M, a_{M}$ is the poldrizabilily of $M$, and $i$ is the internuclear distance between $M$ and $\mathrm{A}^{+}$.

FER also assume that every collision between $\mathrm{MA}^{+}$and $\mathrm{B}^{-}$ leads to reaction and that the steric factor is unity, so that the rate constant for Reaction (F-2) is

$$
\therefore \mathrm{k}_{3}=Q\left\{\frac{8 \mathrm{k}_{B^{T}}}{\pi} \frac{\left(\mathrm{M}_{A^{+}}+\mathrm{M}_{B^{-}}+M_{M^{2}}\right)}{\left(M_{A^{+}}+M_{M^{\prime}}\right) M_{B^{-}}}\right\}^{1 / 2},
$$

where $M_{A^{+}}, M_{B^{-}}$, and $M_{M}$ are the masses of $A^{+}, B^{-}$, and $M$, and $Q$ is the effective cross section. FER arbitrarily choose $Q$ so that the impact parameter has the same value as the distance at which the Coulomb attractive energy is equall to the average thermal energy, $3 / 2 \cdot k_{B} T$, sn tha.t.

$$
Q=4 \pi e^{4} /\left(9 k_{B}^{2} T^{2}\right)
$$

I'able XI gives the values of the pulential parantete's ealeulated by the FER method for $\mathrm{NO}^{+}-\mathrm{M}$ complexes for the different $\mathrm{M}$ gases used. Notice that for $\mathrm{H}_{2}, \mathrm{D}_{2}$, and $\mathrm{He}, \epsilon_{\mathrm{v}}$ is about half as large as $\epsilon_{\mathrm{m}}$, so that for these cases, the harmonic-oscillator approximation is probably in error.

FER consider that complex formation with the positive ion is the dominant process, but we have also done the calculation for $\mathrm{NO}_{2}^{-} \mathrm{M}$ complexes, and Table XII gives these potential parameters. Table XIII contains the values of the rate constant of the exchange reaction, $k_{3}$ and the equilibrium constant for complex formation for both $\mathrm{NO}^{+}-\mathrm{M}$ 
Table XI. Potential parameters for the $\mathrm{NO}^{+}-\mathrm{M}$

\begin{tabular}{|c|c|c|c|c|c|c|}
\hline Gas & $\begin{array}{c}{ }^{\sigma} \mathrm{NO}^{+}-\mathrm{M}^{\mathrm{a}} \\
(\mathrm{A})\end{array}$ & $\begin{array}{c}\epsilon_{{ } \mathrm{NO}^{+}-\mathrm{M} / \mathrm{k}_{\mathrm{B}}{ }^{\mathrm{a}}} \\
\left({ }^{\circ} \mathrm{K}\right)\end{array}$ & $\begin{array}{c}a_{M} \times 10^{24} \cdot . \\
\left.i \mathrm{~cm}^{3}\right)\end{array}$ & $\begin{array}{l}{ }_{. m} \\
(\mathrm{~A})\end{array}$ & $\begin{array}{c}\epsilon_{\mathrm{m}} \times 10^{13} \\
(\mathrm{erg})\end{array}$ & $\begin{array}{r}\epsilon_{\mathrm{v}} \times 10^{14} \\
(\mathrm{erg})\end{array}$ \\
\hline $\mathrm{He}$ & 2.89 & 35.1 & 0.204 & 2.86 & 0.335 & 2.07 \\
\hline $\mathrm{H}_{2}$ & 3.13 & 66.7 & 0.789 & 2.99 & 0.986 & 4.73 \\
\hline $\mathrm{D}_{2}$ & 3.13 & 66.7 & 0.775 & 2.99 & 0.983 & 3.45 \\
\hline $\mathrm{N}_{2}$ & 3.50 & 105 & 1.76 & 3.38 & 1.39 & 1.76 \\
\hline Ar & 3.37 & 120 & 1.63 & 3.26 & 1.50 & 1.77 \\
\hline $\mathrm{Kr}$ & 3.47 & 147 & 2.46 & 3.35 & 2.02 & 1.70 \\
\hline $\mathrm{Xe}$ & 3.70 & 162 & 4.00 & 3.53 & 2.58 & 1.73 \\
\hline
\end{tabular}

"a From reference 32, except that the value of $\sigma_{\mathrm{NO}^{+}-\mathrm{He}}$ is $0.05 \AA$ smaller. 
Table XII. Potential parameters for the $\mathrm{NO}_{2}^{-}-\mathrm{M}$ interaction

\begin{tabular}{|c|c|c|c|c|c|}
\hline Gas & $\begin{array}{c}\mathrm{NO}_{2}{ }^{-}-\mathrm{M}^{\mathrm{a}} \\
(\AA)\end{array}$ & $\begin{array}{c}\mathrm{Co}_{\mathrm{NO}_{2}}-\mathrm{M}^{\mathrm{k} \mathrm{k}_{\mathrm{B}}^{\mathrm{a}}} \\
\left({ }^{\circ} \mathrm{K}\right)\end{array}$ & $\begin{array}{l}\mathrm{r} \mathrm{m} \\
(\stackrel{\AA}{\mathrm{A}})\end{array}$ & $\epsilon_{\mathrm{m}^{\times 10^{13}}}$ & $\begin{array}{c}\epsilon_{\mathrm{v}} \times 10^{14} \\
\vdots(\mathrm{erg})\end{array}$ \\
\hline $\mathrm{He}$ & 3.41 & 47.4 & 3.58 & 0.223 & 1.21 \\
\hline $\mathrm{H}_{2}$ & 3.62 & 90.2 & 3.69 & 0.54 & 2.93 \\
\hline $\mathrm{D}_{2}$ & 3.62 & 90.2 & 3.69 & 0.54 & 2. 11 \\
\hline $\mathrm{N}_{2}$ & 3.99 & 142 & 4.10 & 0.82 & 1.03 \\
\hline Ar & 3.86 & 162 & 3.98 & 0.87 & 1.14 \\
\hline $\mathrm{Kr}$ & 3.96 & 199 & 4.06 & 1.17 & 0.95 \\
\hline $\mathrm{Xe}$ & 4.19 & 220 & 4.24 & 1.50 & 1.15 \\
\hline
\end{tabular}

${ }^{a}$ From Reference 32 where one uses $\sigma_{\mathrm{NO}_{2}^{-}-\mathrm{M}^{-}}=0.5\left(\sigma_{\mathrm{NO}_{2}}+\sigma_{\mathrm{M}}\right)$ with $\sigma_{\mathrm{NO}_{2}}=4.3 \AA$ and $\epsilon_{0 \mathrm{NO}_{2}-\mathrm{M}^{-}}=\left(\epsilon_{0 \mathrm{NO}_{2}} \epsilon_{\mathrm{O}_{\mathrm{M}}}\right)^{1 / 2}$ with $\epsilon_{0 \mathrm{NO}_{2}}=220 \mathrm{k}_{\mathrm{B}}$ (the $\sigma_{\mathrm{NO}_{2}}$ and $\epsilon_{0} \mathrm{NO}_{2}$ are taken as approximately equal to the values for $\mathrm{N}_{2} \mathrm{O}$ ). 
Table XIII. Calculated values of $\mathrm{k}_{3}$ and $\mathrm{K}$ for $\mathrm{NO}^{+}-\mathrm{M}$ and for $\mathrm{NO}_{2}^{-}-\mathrm{M}$ interactions

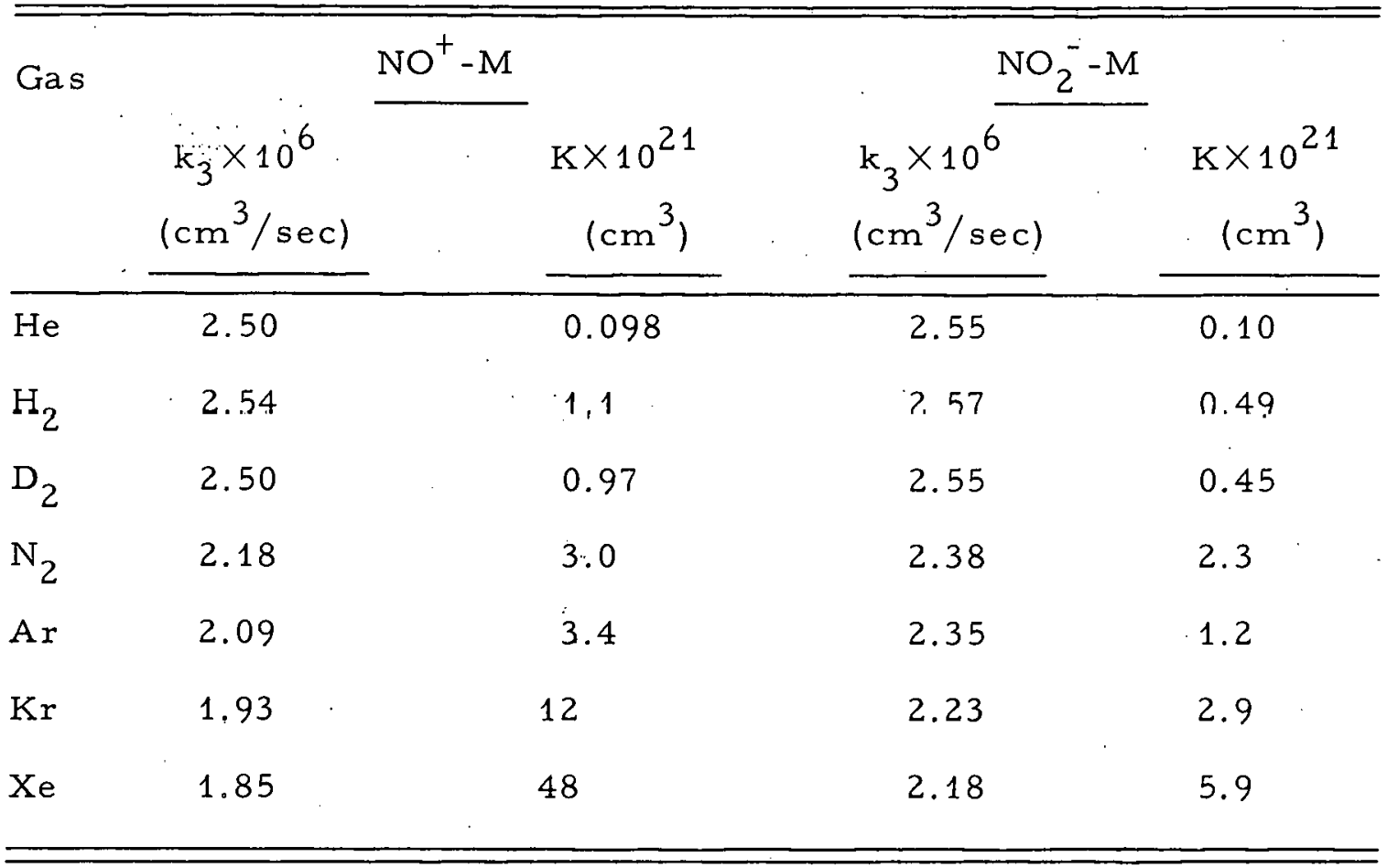


complexes and $\mathrm{NO}_{2}^{-}-\mathrm{M}$ complexes. Column one of Table XIV gives the values of the three-body rate constant, $\mathrm{k}_{\mathrm{T}}$, calculated by assuming only $\mathrm{NO}^{+}-\mathrm{M}$ complexes contribute, and column two gives the relative third --.body efficiencies obtained by taking the ratio of $\mathrm{k}_{\mathrm{T}}$ for each $M$ gas to the valuc of $k_{T}$ for helium. Columns three and four of Table XIV contain $k_{T}$ and the relativc third-body efficiencies calculated by assuming that $k_{T}$ is the sum of the rate constant $k_{T P}$ for $\mathrm{NO}^{+}-\mathrm{M}$ complexes and the rate constant $k_{\mathrm{TN}}$ for $\mathrm{NO}_{2}{ }^{-} \mathrm{M}$ complexes. That is,

$$
\begin{gathered}
\mathrm{NO}^{+}+\mathrm{M} \stackrel{\mathrm{KP}}{\rightarrow} \mathrm{MNO}^{+}+\mathrm{NO}_{2}^{-} \stackrel{\mathrm{k}_{3 \mathrm{P}}}{\longrightarrow} \text { Neutrals, } \\
\mathrm{k}_{\mathrm{TP}}=\mathrm{K}_{\mathrm{P}} \mathrm{k}_{3 \mathrm{P}} ; \\
\mathrm{NO}_{2}^{-}+\mathrm{M} \stackrel{\mathrm{K}_{\mathrm{N}}}{\rightarrow} \mathrm{MNO}_{2}^{-}+\mathrm{NO}^{+} \stackrel{\mathrm{k}_{3 \mathrm{~N}}}{\longrightarrow} \text { Neutrals, } \\
\mathrm{k}_{\mathrm{TN}}=\mathrm{K}_{\mathrm{N} \mathrm{k}_{3 \mathrm{~N}}} .
\end{gathered}
$$

Also included in Table XIV are the experimentally obtained values of $\mathrm{k}_{\mathrm{T}}$ and the relative third-body efficiencies.

The values of $k_{T}$ calculated by using the FER theory are generally below the experimental values; this is especially true for our experiments except for the case of xenon. The predicted order of thirdbody efficiencies of

$$
\mathrm{Xe}>\mathrm{Kr}>\mathrm{Ar} \approx \mathrm{N}_{2}>\mathrm{H}_{2} \approx \mathrm{D}_{2}>\mathrm{He}
$$

is qualitatively correct, except for the predictions of $\mathrm{Kr}>\mathrm{Ar} \approx \mathrm{N}_{2}$ and $\mathrm{H}_{2} \approx \mathrm{D}_{2}>\mathrm{He}$.where the experimental results are $\mathrm{N}_{2} \approx \mathrm{Kr} \approx \mathrm{Ar}$ and $\mathrm{D}_{2} \approx \mathrm{H}_{2} \approx \mathrm{He}$. However, the calculation predicts a much greater variation in third-body efficiencies than is observed; the ratio of $\left(k_{\mathrm{T}}\right)_{\mathrm{XE}} /\left(\mathrm{k}_{\mathrm{T}}\right)_{\mathrm{He}}$ is in error by a factor of 30 to 50.

The calculated values are fairly sensitive to the values of $\sigma$ used, and give larger values of $\mathrm{K}$ and $\mathrm{k}_{\mathrm{T}}$ for smaller values of $\sigma$. Therefore, the calculated values could probably be brought into better agreement with the experimental values by using smaller values of $\sigma$ 
Table XIV. Comparison of calculated values of $k_{T}$ and relative third-body efficiencies with observed values

\begin{tabular}{|c|c|c|c|c|c|c|}
\hline$\underline{\text { Gas }}$ & $\begin{array}{l}\mathrm{k}_{\mathrm{TP}}^{\mathrm{a}} \times 10^{26} \\
(\mathrm{~cm} 6 / \mathrm{sec})\end{array}$ & $\frac{\left(\mathrm{k}_{\mathrm{TP}}\right)_{\mathrm{M}}}{\left(\mathrm{k}_{\mathrm{TP}}\right)_{\mathrm{He}}}$ & $\begin{array}{c}\mathrm{k}_{\mathrm{TP}+\mathrm{N}^{\mathrm{b}}} \times 10^{26} \\
\left(\mathrm{~cm}^{6} / \mathrm{sec}\right)\end{array}$ & $\frac{\left(k \mathrm{TP}+\mathrm{N}^{\prime} \mathrm{M}\right.}{\left(\mathrm{k}_{\mathrm{TP}+N^{\prime}}\right)_{\mathrm{He}}}$ & $\begin{array}{l}\mathrm{k}_{\mathrm{T}} \times 10^{26} \\
\text { Expt. } \\
\left(\mathrm{cm}^{6} / \mathrm{sec}\right)\end{array}$ & $\frac{\left(k_{T}{ }^{\exp t}\right)_{M}}{\left(k_{T}{ }^{\exp t_{1}}{ }_{H e}\right.}$ \\
\hline $\mathrm{He}$ & 0.024 & 1.0 & 0.050 & 1.0 & 4.1 & 1.00 \\
\hline $\mathrm{H}_{2}$ & 0.28 & 12 & 0.41 & 8.1 & 5.8 & $1.4 \pm 0.4$ \\
\hline $\mathrm{D}_{2}$ & 0.24 & 10 & 0.36 & 7.1 & 6.1 & $1.5 \pm 0.4$ \\
\hline $\mathrm{N}_{2}$ & 0.66 & 27 & 1.2 & 24 & 21 & $5.2 \pm 1.1$ \\
\hline Ar & 0.71 & 29 & 0.98 & 20 & 14.5 & $3.6 \pm 0.8$ \\
\hline $\mathrm{Kr}$ & 2.3 & 94 & 4.4 & 87 & 17.5 & $4.3 \pm 1.0$ \\
\hline $\mathrm{Xe}$ & 8.9. & 360 & 10.0 & 200 & 28 & $6.8 \pm 1.5$ \\
\hline $\mathrm{b}_{\mathrm{k}}$ & $\begin{array}{l}\left(\mathrm{k}_{3} \mathrm{~K}\right) \mathrm{NO}^{+} \\
=\left(\mathrm{k}_{3} \mathrm{~K}\right)_{\mathrm{NC}}\end{array}$ & $+\left(k_{3} K\right.$ & & & . & \\
\hline
\end{tabular}


and adjusting the $\sigma^{\prime} s$ to reduce the differences in relative third-body efficiencies. However, the value of $\mathrm{k}_{\mathrm{T}}$ for the $\mathrm{NO}^{+}-\mathrm{He}$ complex has already been increased somewhat by using a value of $\sigma_{\mathrm{He}}$ which is $0.10 \AA$ smaller than the accepted value, ${ }^{32}$ and even then, the calculated value for $\left(k_{T}\right)$ He seems especially low.

Another feature of the FER theory is the calculation of $4.4 \times 10^{-11} \mathrm{~cm}^{2}$ for the effective cross section, $Q$, of Reaction (F-2). Sincc all the calculated values of $k_{T}$ are low, it could be argued that FER's arbitrary choice of the definition of $Q$ gives values of $Q$ that are too low. Huwever, even lliese values if $Q$ aie so large that the mech anism seems unreasonable, as indicated in the following argument: The value of $4.4 \times 10^{-11} \mathrm{~cm}^{2}$ implies that the average distance of closest approach is about $190 \AA$. It is difficult to see why the $M-A^{+}$ complex should break up under the influence of an ion $190 \AA$ away. Even if the $A B$ formed in Reaction $(F-2)$ is considered to be an unneutralized ion pair whose relative kinetic energy is so low that the ions cannot separate to large distances, the $M$ must carry off an extra energy of around $k_{B} T$, and it seems strange that the $M$ should be so violently ejected under the influence of an ion so far away. (If we assume that the $A B$ is a neutral molecule, then the FER theory is even more difficult to believe, since it would imply that the $M$ has removed the several electron volts of energy evolved in charge neutralization.) This is in contrast to the situation in the atom-recombination problem, because . the distances involved in atom-recombination reactions are more nearly on the order of molecular sizes.

Because of the repeated failures of the quantitative predictions and because of the difficulties discussed above, it seems that the FER theory gives only a very rough picture of the charge-neutralization process. Therefore, in view of the more consistent agreement of the collisional-deactivation mechanism presented next, it appears that for ion-ion recombination the mechanism of complex formation followed by exchange reaction is not as good an explanation as the collisionaldeactivation mechanism. This is in contrast with atom recombination, in which the complex-formation mechanism ${ }^{11}$ has several advantages over the collisional-deactivation mechanism. 


\section{B. Thomson Theory}

In the pressure region used for these experiments, the theory that has generally been used for ion-ion recombination in the volume was proposed by J. J. Thomson in $1924 .^{8,38}$ Thomson suggested that the increase in the recombination coefficient $a_{T}$ with increasing pressure could be explained if it were assumed that (a) ion-charge neutralization is not likely to occur during one collision of the ions and (b) ions that undergo collisions with the neutral gas molecules while the ions are fairly close together (where their kinetic energies are above the thermal energy of the neutrals) may lose enough of their kinetic energy of relative motion so that they become unable to separate to infinity. Because the ions cannot separate to infinity after they first pass through their distance of closest approach, they form a bound ion pair in which they oscillate between the minimum and maximum separations until charge neutralization eventually occurs. This mechanism is essentially one of collisional deactivation of an excitedion pair to form an ion pair in a bound state, where the ions eventually neutralize each other.

Thomson assumes that the impact parameter that corresponds to the distance within which the ions are deactivated on an average collision with a neutral is given by

$$
B_{T}=(2 / 3) e^{2} / k_{B} T
$$

where $k_{B}$ is the Boltzman constant, $T$ is the absolute temperature, and $e$ is the electronic charge. Then, the three-body recombination coefficient, $a_{T}$, is given by

$$
a_{T}=\pi B_{T}^{2} w\langle g\rangle
$$

where $W$ is the probability, that one of the ions will undergo a collision with a neutral while the ions are inside the critical region whose impact parameter is $B_{T}$, and $\langle g\rangle$ is the mean, relative velocity at infinite ion separation.

The factor $W$ is then calculated by assuming that the ions follow straight-line trajectories through the critical region (ignoring 
the actual curvature caused by the attractive potential). When the positive and negative ions have equal masses and equal mean free paths for collisions with the neutrals, $\lambda$, then $W$ is calculated as a function of a parameter $X$, defined as

$$
\mathrm{X}=\mathrm{B} / \lambda \text {, }
$$

where $B$ is defined the same as $B_{T}$ in Eq. $(F-8)$, although here $B$ is considered to be the actual radius of the critical region instead of the impact parameter that brings an ion into the critical region. For $X>>1$, we have $W=1$, for $X<<1, W=8 x / 3$, and for intermediate values of $X$, the values of $W$ are tabulated by Loeb. ${ }^{39}$ Since $\lambda$ is inversely proportional to the pressure, $\mathrm{X}$ is directly proportional to llie pressure. Also, since $W$ is the only pressurc-dependent torm in Eq. (F-9), the shape of the curve of $a_{T}$ vs pressure is the same as the shape of the curve of $W$ vs $X$, which is given in Fig. 20. This general form of the dependence of $a_{T}$. on pressure was confirmed by the experimental work of Gardne ${ }^{4}$ and Sayers. 5

\section{Natanson Theory}

At some pressure above 1 to $2 \mathrm{~atm}$, a ceases to increase with increasing pressure, and a eventually decreases with increasing pressure. In this high-pressure region the rate determining step is the rate at which the ions approach each other; the recombination coefficient is determined by the ionic mobilities and is given by the Langevin expression, 40

$$
a=4 \pi e\left(k_{+}+k_{-}\right),
$$

where $k_{+}$and $k_{\text {_ }}$ are the mobilities of the positive and negative ions and are inversely proportional to the pressure. Natanson has extended Thomson's theory to give a single equation which goes from a form similar to Eq. $(F-9)$ at low pressures to a form similar to Eq. (F-11) at high pressures. 9

Natanson uses somewhat different requirements for the produc:tion of an ion pair in a bound state; we now discuss these requirements. Natanson assumes that when the two ions are separated at infinity, they 
$-77-$

,

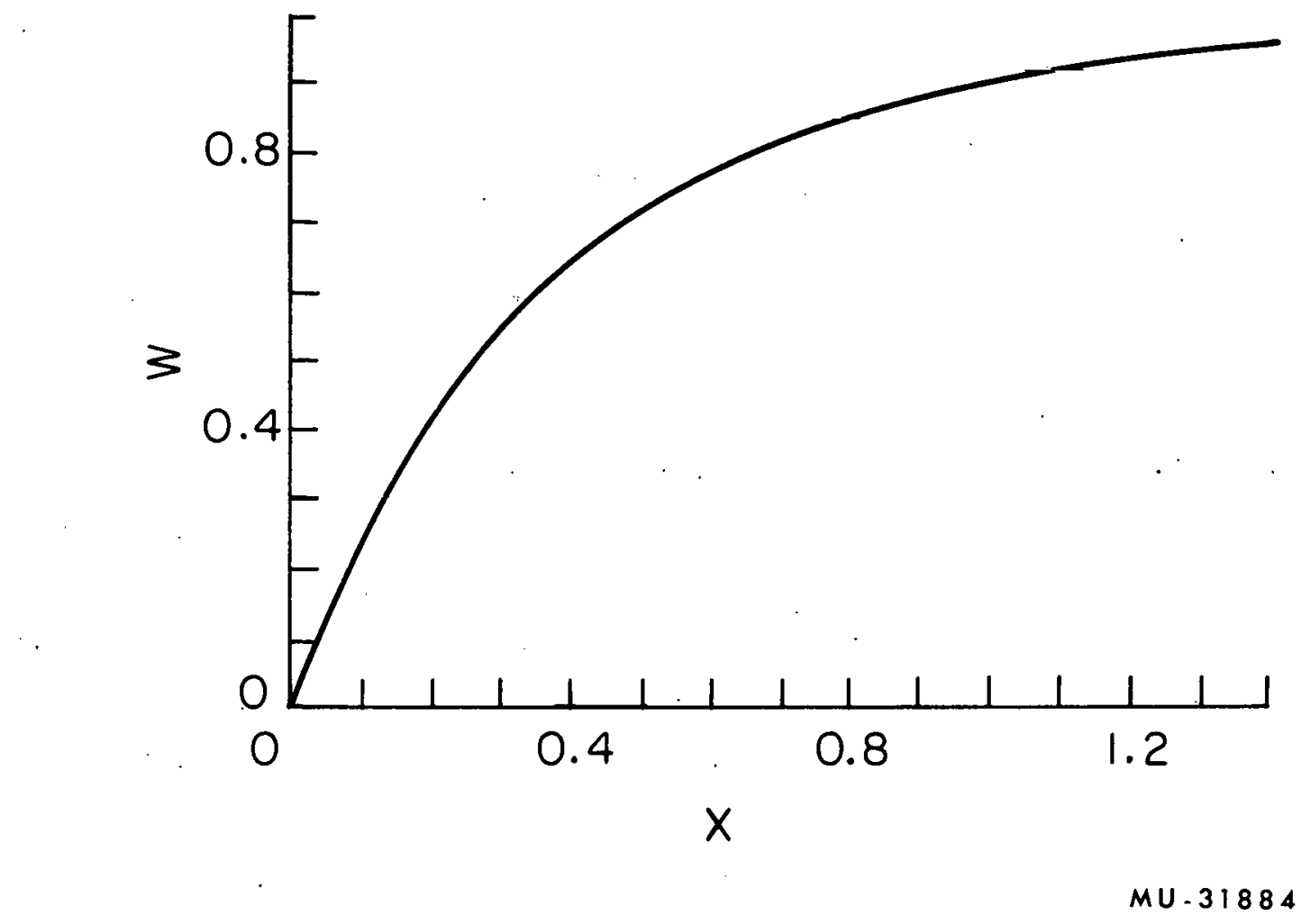

Fig. 20. A plot of $W$ vs $X$. This curve gives the general shape of the a vs $P$ plots using the Thomson theory. 
have a kinetic energy of relative motion, $T_{R^{\prime}}(\infty)$, of $(3 / 2) k_{B} T$ and that the kinetic energy of the motion of the center of mass, $T_{C}$, is also $B / 2) k_{B} T$. Further, when two ions of equal mass are a distance $\rho$ apart, he assumes that the kinetic energy of each ion is

$$
T_{1}(\rho)=T_{2}(\rho)=\frac{3}{\overline{2}} \dot{k}_{B} T+\frac{e^{2}}{2 \rho} .
$$

If ion number one has a collision with a neutral while separaterl a distance $\rho$ from the second ion. thril thr energy of the occond iun is aosuried lu remain unchanged, while the energy of ion number one be:comes, on the average,

$$
\begin{aligned}
T_{1}^{i^{\prime}}(\rho) & =\frac{1}{2}\left(T_{1}+{ }^{\prime}{ }^{\prime}\right) \\
& =\frac{3}{2} k_{B} T+\frac{e^{2}}{4 \rho}
\end{aligned}
$$

where $T_{M}=\frac{3}{2} k_{B} T$ is the kinetic energy of the neutral. Equation $(F-13)$ is for ions and neutrals with equal masses; it also contains the assuriplion that the ion-neutral collision is similar to a hard-sphere collision in order that $T_{1}^{\prime}=1 / 2\left(T_{1}+T_{M}\right)$. Natanson now assumes that after the ion-neutral collision, all directions of the velocity of ion one arc equally prububle. Under this assumption, the mean kinetic energy of the relative ion motion after the collision, $T_{R^{\prime}}^{\prime}$, becomes

$$
T_{R^{\prime}}(\mu)=\frac{1}{2}\left[I_{1}^{\prime}:(\rho)+I_{2}^{\prime}(\rho)\right]=\frac{3}{2} k_{B} T+\frac{3}{8} \frac{e^{2}}{\rho} \text {. }
$$

Further, Natanson requires that the ion pair must:lose enough relative kinetic energy so that the ions are unable to separate to a distance greater than $B+\beta \lambda$, where $\beta$ has a value on the order of unity, $\lambda$ is the mean free path, and $B$ is the radius of the region in which deactivation occurs on an average collision and is defined by

$$
T_{R}^{\prime}:(B) \leqslant \int_{B}^{B+\beta \lambda} F d r
$$


The reason for this more stringent requirement is that when the ions are rather far apart, a further collision with a neutral may increase the kinetic energy of the ion pair and allow the ions to separate to infinity.

Then, Natanson assumes that the energy of the ions at $B+\beta \lambda$ is $(3 / 2) k_{B} T$, so the expression for $B$ is

$$
B=(\beta \lambda / 2)\left[\left(1+5 e^{2} / 3 k_{B} T \beta \lambda\right)^{1 / 2}-1\right] \text {. }
$$

In Natanson's theory, free flight occurs between $B$ and $B+\beta \lambda$, so that the impact parameter at $B+\beta \lambda$ is related to $B$ by

$$
[b(B+\beta \lambda)]^{2}=B^{2}\left(1+1 / T_{R}(B+\beta \lambda)\right) \int_{B}^{B+\beta \lambda} F d r
$$

The velocity distribution at $B+\beta \lambda$ is assumed to be a Maxwellian distribution with the result that

$$
[b(B+\beta \lambda)]^{2}=B^{2}(1+12 / b)=(1 / / b) B^{2} .
$$

Natanson determines the concentration of ions at $B+\beta \lambda$ to take account of the loss of ions by recombination inside $B$. The expression for the ratio of $n_{B+\beta \lambda}$ to the concentration in the volume is

$$
\begin{gathered}
\frac{{ }^{n} B+\beta \lambda}{n}=\exp \left[\frac{e^{2}}{(B+\beta \lambda) k_{B} T}\right] \cdots \\
\left.\left\{1+\frac{B^{2}\langle y\rangle \cdot{ }^{W} k_{B} T}{\cdots} \cdot \frac{e^{2} \beta \lambda}{B(B+\beta \lambda) k_{B} T}\right] \cdot\left[\exp \left(\frac{e^{2}}{\left.(B+\beta \lambda) k_{B} T\right)}\right)-1\right]\right\}^{-1},
\end{gathered}
$$

where $\mathrm{D}=\mathrm{D}_{+}+\mathrm{D}_{-}$and $\mathrm{D}_{+}$and $\mathrm{D}_{\text {- }}$ are the diffusion coefficients of the positive and negative ions, respectively.

By using ${ }^{n} B+\beta \lambda$ ' taking $\beta=1$, and making the substitution of $X=B / \lambda$, one obtains the final result 


$$
a_{T}=\frac{17}{5} \pi\langle g\rangle w \lambda^{2} x^{2} \exp (2 X)\left\{1+\frac{17}{20}\langle g\rangle k_{B} \frac{T w \lambda^{2} X^{2}}{e^{2} D}[\exp (2 X)-1]\right\}^{-1}
$$

If Natanson, in deriving Eq. $(F-16)$, had taken the ion energy at $B+\beta \lambda$ to be something other than $(3 / 2) k_{B} T$, then the exact value of $a_{T}$ would change somewhat.

When the pressure is low enough that $\lambda>>_{e}^{2} /\left(k_{B} T\right)$ then Eq. $(F-20)$ becomes

$$
a_{T}=(17 / 5) \pi x^{2} \lambda^{2} w\langle g\rangle \exp \left(e^{2} / \lambda k_{B} T\right) \approx(17 / 5) \ldots x^{2} \lambda^{2} w\langle g\rangle
$$

'I'o predict $a_{r}$ ', we must know the mean free path, $\lambda$. Loeb has suggested that $\lambda$ may be evaluated from the ionic mobility, $k$, by using the relationship 39

$$
\lambda=\frac{k M_{M} C(300)}{0.815 e}\left(\frac{M_{I}}{M_{M}+M_{I}}\right)^{1 / 2}
$$

where $M_{I}$ and $M_{M}$ are the masses of the ions and the neutrals, $C$ is the root-mean-square velocity of the neutrals, and $\mathrm{k}$ is in $\mathrm{cm}^{2} /(\mathrm{V} \mathrm{sec})$. In any case, one must know the mobility in order to fistimalip the diffusion coefficient. Also, the mobility can be estimated from the calculations by Langevin 28 and Hassé29 as described in Sec. IV. So, if the masses of the ions are known and the mobilities can be estimated, then $a_{T}$ can be evaluated from Eqs. $(F-20)$ or $(F-21)$.

\section{Modifications Required When the Ion Mass and the Neutral Mass are Unequal}

Because in these experiments we study the effect of using different neutral molecules as the third bodies, we need to consider how different masses of the neutrals affect the predicted values of $a_{T}$. In the zero-order approximation we say that if the mass of the neutral is not much different from the mass of the ion then we may still consider the masses to be equal. Then, we can predict the relative third-body 
efficiencies by comparing the predicted values of $a_{\mathrm{T}}$ at low pressures, as given by Eq. (F-21). Further, if the pressure is low enough, then $\mathrm{X}=\mathrm{B} / \lambda<1$, so that $\mathrm{W}=8 \mathrm{X} / 3$, and we may approximate $\mathrm{B}$ by.

$5 / 12\left(e^{2} / k_{B} T\right)$ ! Then Eq. $(F-21)$ becomes

$$
a_{T}=(136 / 15) \pi\left(5 e^{2} / 12 k_{B} T\right)^{3}(1 / \lambda)\langle g\rangle
$$

Taking ratios of $a_{T}$ to get relative third-body efficiencies, we obtain

$$
\left(a_{T}\right)_{M} / /\left(a_{T}\right)_{M}=(\lambda)_{M} /(\lambda)_{M}^{8} \approx\left(a_{M}{ }^{8 / /} a_{M}\right)^{1 / 2}
$$

where $\left(a_{T}\right)_{M}$ is the value of $a_{T_{i}^{\prime}}$ evaluated for the neutral $M$ and where $a_{M}$ is the polarizability of $M$. The second approximation used in Eq. $(F-24)$ is that the mean free path is inversely proportional to the square root of the polarizability, which is a fairly good approximation if the neutral is more polarizable than helium, as can be seen in Sec.IV.

Equation (F-24) cannot be expected to be correct over a large $\ldots:$ range of neutral masses; therefore, let us now consider some of the main effects when we change the neutrals. One effect is that because of the difference in the masses the ion can not transfer all its energy to the neutral in one collision. The exp'ression for the maximum a... amount of energy that can be lost by the ion is in a simple form if the neutral is initially at rest, in which case

$$
\left(\Delta T_{1}\right)_{\max }=\frac{4 M_{I} M_{M}}{\left(M_{1}+M_{M}\right)^{2}} T_{1}=\gamma T_{1} .
$$

where $M_{1}$ and $M_{M}$ are the masses of the ions and the neutrals. ${ }^{41}$ In a real situation the neutrals are not initially at rest; however, it may be a reasonable approximation to consider that Eq. (F-25) holds for the energy that the ion has in excess of the kinetic energy of the neutral, 42 so that

$$
\left(\Delta T_{1}\right)_{\max }=\gamma\left(T_{1}-T_{M}\right)=\frac{4 M_{1} M_{M}}{\left(M_{1}+M_{M}\right)^{2}}\left(T_{1}-T_{M}\right)
$$


This approximation can be used only for the neutral gases that are not much lighter than the ions, because the ion velocity must be larger than the neutral velocity. Another difficulty arises if the neutral is lighter than the ion, because then the maximum scatteringangle of the velocity factor of ion one becomes lcss than $90^{\circ}$ so that not all directions are possible for $V_{m}^{\prime}$, and the assumption of equal probability for all possible directions of $V_{m}^{\prime}$ ' becomes worse as the mass of the neiutral decreases $^{43}$ (even when the masses are equal, not all directions are equally probable).

However, considering only the cases in which the ion mass is only slightly larger than the neutral mass, we may use $\left(\Delta T_{1}\right)_{\text {max }}$ in the derivation of $B$ as a rough approximation of the effect of changing the neutral mass. By the same arguments as before, Eqs. $(F-14)$ and $(F-16)$ become

and

$$
T_{R}^{\prime}:(\rho)=\frac{3}{2} k_{B} T+\frac{e^{2}}{8 \rho}(4-\gamma)
$$

$$
B=\frac{\beta \lambda}{2}\left[\left(1+\left(\frac{4+\gamma}{3}\right) \frac{e^{2}}{k_{B}^{T \beta \lambda}}\right)^{1 / 2}-1\right] .
$$

Thus, Eq. $(F-20)$ is modificd by a different value of $X$ [since $B$ is now detined by E'q. (F-27)], and also the $17 / 5$ and $17 / 20$ terms are replaced by $(16+\gamma) /(4+\gamma)$ and $(16+\gamma) /[4(4+\gamma)]$. Equation $(F-23)$ for the very low pressure. is now

$$
a_{T}=\frac{8}{3}(16+\gamma) \pi(4+\gamma)^{2}\left(\frac{e^{2}}{12 k_{B} T}\right)^{3}\left(\frac{1}{\lambda}\right)\langle g\rangle,
$$

and the relative third-body efficiencies are given by

$$
\begin{aligned}
& \frac{\left(a_{T^{\prime}} M^{\prime}\right.}{\left(a_{T^{\prime}} M\right.}=\frac{(\lambda)_{M}}{(\lambda)} \frac{\left\{(16+\gamma)(4+\gamma)^{2}\right\}^{\prime} M^{\prime}}{\left\{(16+\gamma)(4+\gamma)^{2}\right\}_{M}} \\
\approx & \left(\frac{a_{M^{\prime}}}{a_{M}}\right)^{1 / 2} \frac{\left\{(16+\gamma)(4+\gamma)^{2}\right\}_{M}}{\left\{(16+\gamma)(4+\gamma)^{2}\right\}_{M}} .
\end{aligned}
$$

Table XV gives the relative third-body efficiencies calculated from 
Table XV. Comparison of experimental relative third-body efficiencies with predictions from Eq. (F-30)

\begin{tabular}{|c|c|c|c|c|}
\hline \multirow[t]{2}{*}{$\begin{array}{c}\text { Neutral } \\
\text { gas }\end{array}$} & \multirow[b]{2}{*}{$\gamma$} & \multirow[b]{2}{*}{$(16+\gamma)(4+\gamma)^{2}$} & \multicolumn{2}{|c|}{$\begin{array}{c}\text { Third-body efficiencies } \\
\text { relative to argon }\end{array}$} \\
\hline & & & Predicted & Experimental \\
\hline \multicolumn{5}{|c|}{ Ion Molecular Weight $=46$} \\
\hline Ar & 0.996 & 424 & 1.00 & 1.00 \\
\hline $\mathrm{N}_{2}$ & 0.942 & 414 & 1.01 & $1.44 \pm 0.3$ \\
\hline $\mathrm{Kr}$ & 0.916 & 409 & 1.18 & $1.19 \pm 0.3$ \\
\hline $\mathrm{Xe}$ & 0.769 & 382 & 1.41 & $1.89 \pm 0.4$ \\
\hline \multicolumn{5}{|c|}{ Ion Molecular Weight $=76$} \\
\hline Ar & 0.904 & 407 & 1.00 & 1.00 \\
\hline $\mathrm{N}_{2}$ & 0.787 & 385 & 0.98 & $1.44 \pm 0.3$ \\
\hline $\mathrm{Kr}$ & 0.998 & 425 & 1.28 & $1.19 \pm 0.3$ \\
\hline $\mathrm{Xe}$ & 0.927 & 411 & 1.59 & $1.89 \pm 0.4$ \\
\hline
\end{tabular}


Eq. $(\mathrm{F}-30)$ for $\mathrm{N}_{2}, \mathrm{Ar}, \mathrm{Kr}$, and $\mathrm{Xe}$, along with the experimental values. From the values of $\gamma$ and of the $(16+\gamma)(4+\gamma)^{2}$ terms in Table XV, we see that these terms vary by only $10 \%$ or less, so that Eq. (F-24) would not be too bad an approximation for this range of neutral masses.

Because of the uncertainty already present in Eq. $(F-20)$ caused by the problem of how to choose $T_{R}(B+\beta \lambda)$ and since the values of $\gamma$ are rather close to onc, we used Eq. $(F-20)$ to calculate $a_{T}$ rather than the $\gamma$ corrections outlined above. Also. the positive anrl the sugdtive ions were assumed to have equal masses. With the se assumptions, $a_{T}$ was calculated for various pressures of the different third-body gases. If we a $\varepsilon \varepsilon u m c$ that the two-budy rale of lon recombination is simply added to the termolecular rate, then the predicted values of $a$ are $a=k_{0}+a_{T}$, where $k_{0}=2.1 \times 10^{-7} \mathrm{~cm}^{3} / \mathrm{sec}$, as determined in Sec. V-D. Figures 21 through 24 show the comparison with the experimental data which the predicted values calculated by assuming two different ion masses. On Fig. 22, two curves are plotted for $\mathrm{N}_{2}$, one using $\lambda$ obtained from experimental values of ions in $N_{2}\left(\lambda_{760}=3.9 \times 10^{-6}\right.$ $\mathrm{cm}$, where $\lambda_{760}$ is $\lambda$ when $P=760$ tor $r$ ), and one using $\lambda$ obtained from predictions in Sec. IV.

Otheir improvements should be made in the Natanson theory to give a more realistic picture. One improvement would be to calculate the average amount of energy transferred from the ion to the neutral by using an ion-induced dipole potential for the interaction between the ion and the neutral. Another improvement would be to remove the approximation that all directions of $V_{1}^{\prime}$ àre equally probable (especially, in the case $M_{1} \geqslant M_{M}$ ). However, as can be seen from Figs. 21 through 24, the predicted values of a agree with the experimental values to within a factor of 2 or less, depending upon what the ion mass actually is. So, to get a quick estimate of the value of a at any pressure, the Natanson theory does give fairly good results, and the theory actually seems to work much better than would be expected from considerations of the approximations involved. 


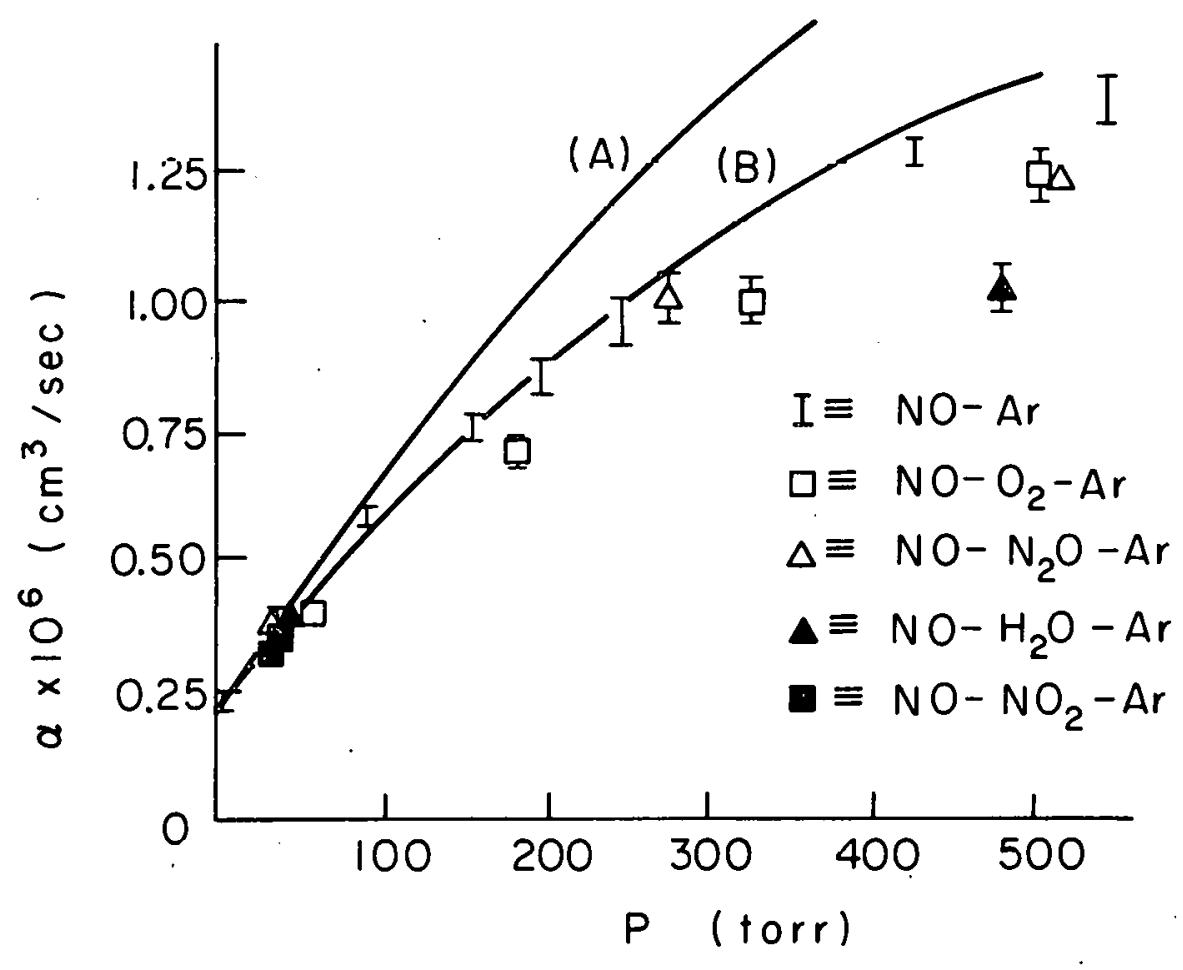

$M U .31885$

Fig. 21. Comparison of the Natanson theory prediction of a vs $P$ with data in $A r$. (A) and (B) are predicted using $\lambda_{760}=440 \AA ; \quad$ (A) ion gram-molecular weight of 46 .

(B) ion gram-molecular weight of 76 . 


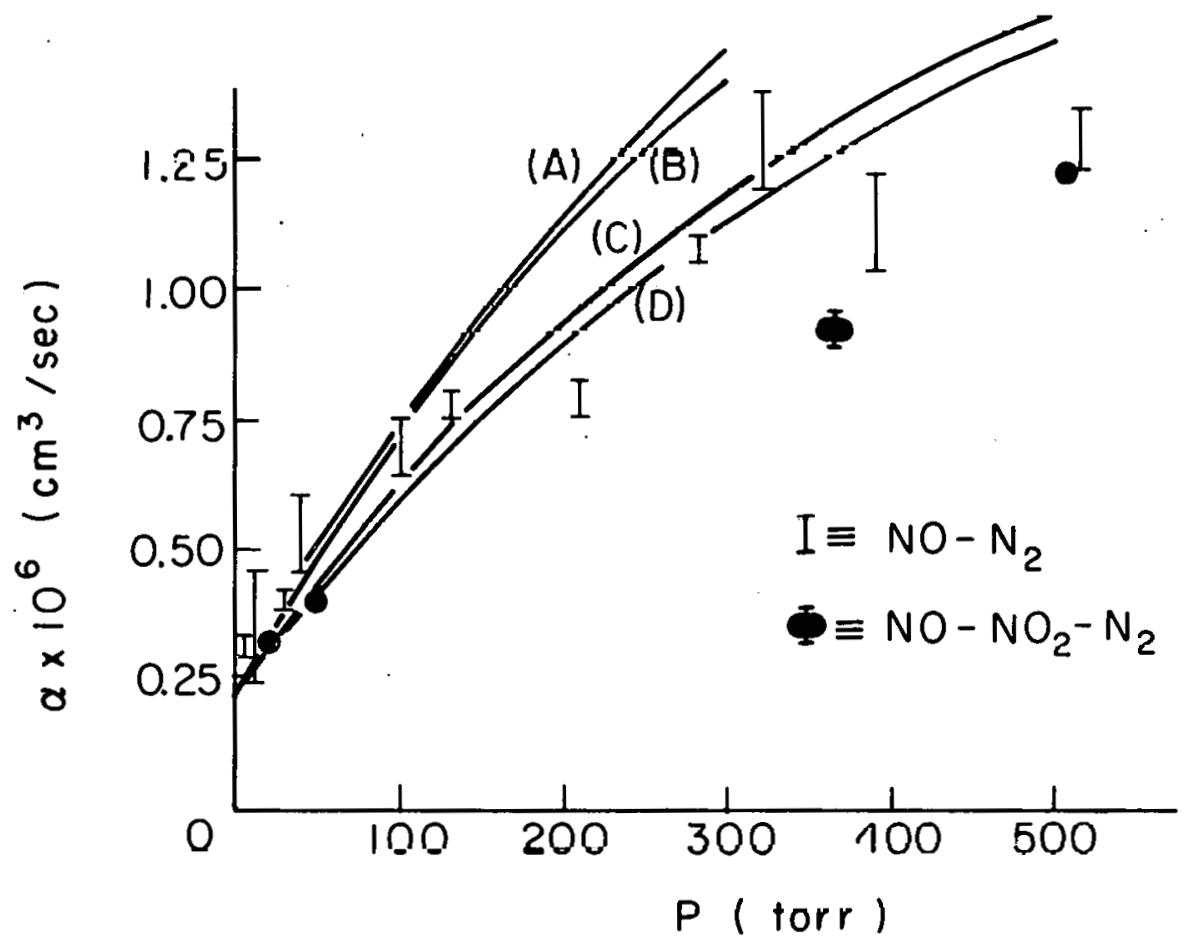

MU. 31886

Fig. 22. Comparjion of the Natanson theory prediction of a vs $P$ with data in $N_{2}$. (A) and (C) are predicted using $\lambda_{760}=420 \AA$. (B) and (D) a re predicted using $\lambda_{760}=390 \AA$. (B) ion gram-molecular weight of 46 . (C) and (D) ion gram-molecular weight of 76 . 


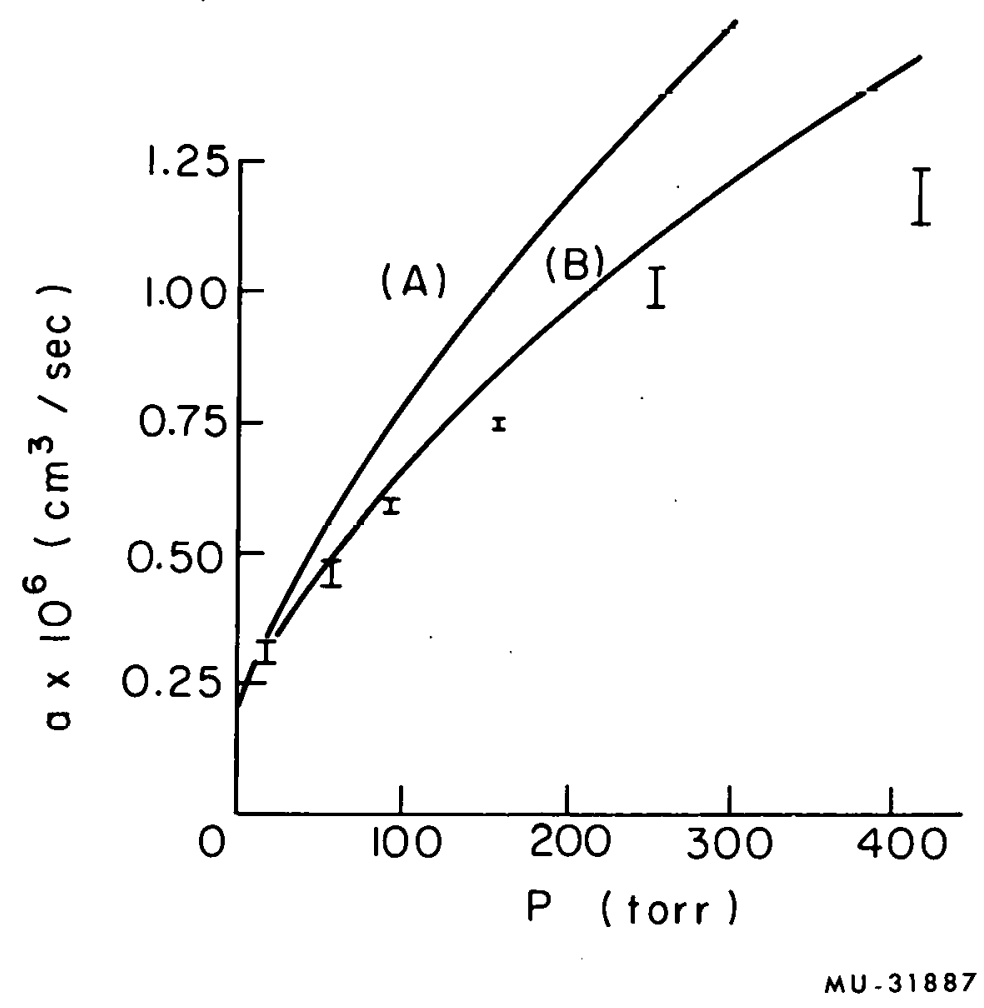

Fig. 23. Comparison of the Natanson theory prediction of a vs $P$ with data in $K r$. (A) and (B) are predicted using $\lambda_{760}=360 \AA$. (A) ion gram-molecular weight of 46 .

(B) ion gram-molecular weight of 76 . 


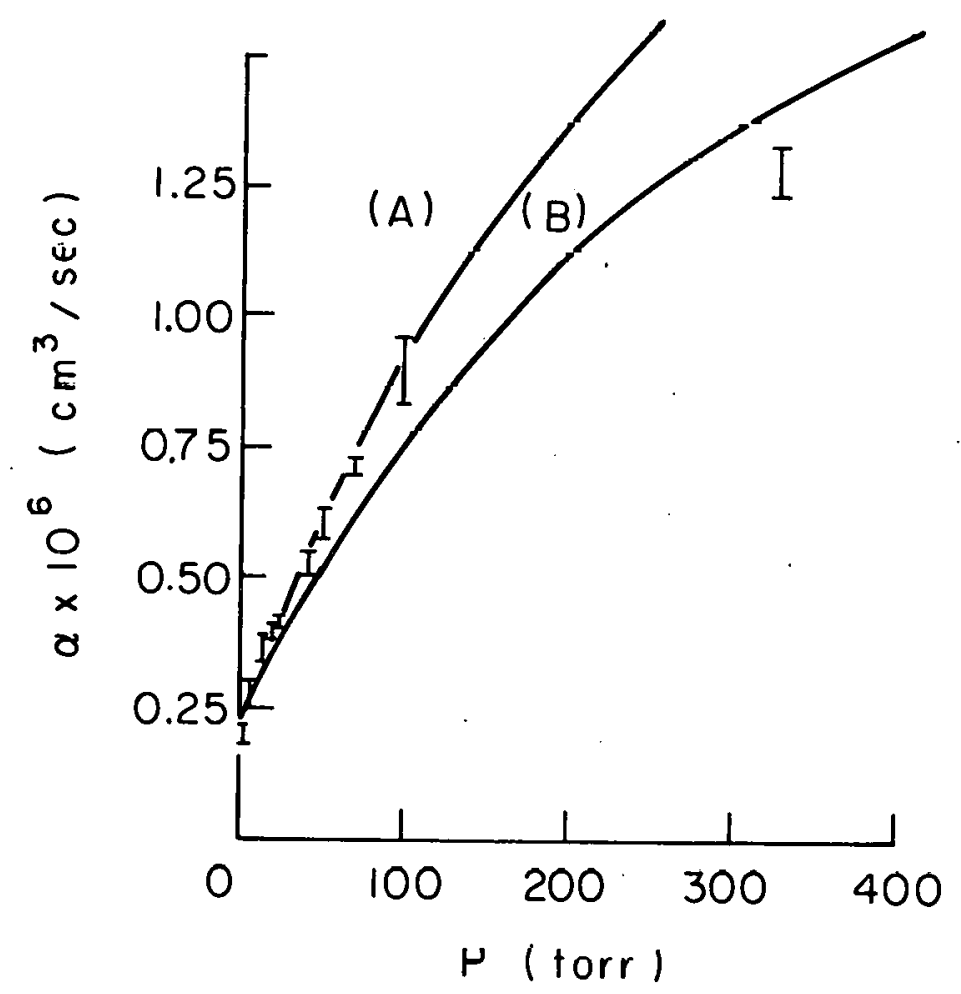

$M U-31888$

Fig. 24. Comparison of the Natanson theory prediction of a vs $P$ with data in Xe. (A) and (B) are predicted using $\lambda_{760}=280 \AA$. (A) ion gram-molecular weight of 46 .

(B) Ion gram-mulecular weight of 76 . 


\section{DETAILED CALCULATION \\ OF THE COLLISIONAL DEACTIVATION MECHANISM}

From Sec. VI we see that the Natanson theory based on the collisional deactivation mechanism gives better results than does the ion-complex formation mechanism of Fueno, Eyring, and Ree. However the Natanson theory contains several approximations that are open to question; also, the theory cannot be expected to (and does not) give good predictions when the mass of the ion is much larger than the mass of the neutral. For these reasons, we decided to do a:more detailed calculation based upon the collisional deactivation mechanism, with the hope of making better predictions of the relative third-body efficiencies; the results of this calculation are discussed in this section.

\section{A. Basic Assumptions}

Because the plots of $1 / \vec{a}_{T}$ are more nearly linear when plotted vs $1 /[\mathrm{M}]$ than when plotted vs $1 /[\mathrm{M}]^{2}$, it seems that only one collision is required for deactivation, and we assume that we may neglect contributions to the ratc of ion-pair deactivation from ion pairs that are $\therefore$ deactivated in more than one collision. Thus, we have a three-body collision between the positive ion, the negative ion, and the neutral gás molecule to consider; we approximate this three-body collision by two two-body collisions: the ion-ion collision and the ion-neutral collision.

The experimental data indicate that the deactivation collision may occur while the ions are tens to hundreds of angstroms apart, so that it would seem to be a good approximation to consider that the neutral collision affects only one of the ions. Thus, we consider that ions number one and number two are following a two-body collision trajectory when ion number one collides with a neutral and this collision changes the velocity vector of ion number one but does not change the velocity vector of ion number two. Further, because the ion-neutral interaction is short-range in comparison with the Coulomb interaction obetween the ions, we make the approximation that the velocity of ion one changes suddenly from $V_{1}(\rho)$ to $V_{1}^{\prime}(\rho)$, where $\rho$ is the ion-ion separation at the time when the ion-neutral separation, $r_{1 \mathrm{M}}$, is minimum, and that the value of $\rho$ remains unchanged during the time of the 
ion-neutral collision. (A partial list of symbols is given at the end of this section.)

Also, we assume that the scattering of ion one is not influenced by the presence of ion two. This approximation neglects the focusing effect that would be expected, because the Coulomb field at ion one from ion two should cause scattering along the direction of $r$ (the ion-ion separation) to be favored. This focusing is most effective when the ions are close together at the time of the collision with the neutral; here the assumption that $V_{2}^{\prime}:(\rho)=V_{m}(\rho)$ is less valid. However, if the ions are close together they are easily deactivated anyhow, because of their large kinetic energy. Therefore the neglect of focusing may not be very serious, because (a) there is already a high probability of deactivation when focusing would be most important, and (b) the region in which the Coulomb energy is much larger than thermal energy is small in comparison with the total region in which deactivation can take place.

The potential between the ions is assumed to be pure Coulombic and the potential between the ions and the neutrals is assumed to be the ion-induced dipole potential with a hard sphere core--the same potential used to calculate the ionic mobility--and the potential is given by Eq. $(D-26)$ in Sec. IV. Further, it is assumed that classical mechanics may be used to describe the collisions, and that the two-body collisions can be treated as central-force problems. Also, we assume that the ion and the neutral have elastic collisions.

Using these basic assimptions, we may now proceed lu calculation of the rate for ion-pair deactivation.

\section{B. Requirements for Deactivation}

When $r=\rho$, the requirement for the ions to be unable to separate to infinity is that their relative kinetic energy, $T_{R}$, be small enough that

$$
T_{R}(\rho) \leqslant \frac{e^{2}}{\rho} .
$$

The value of the relative velocity of the ion pair, $g$, after the ionneutral collision is 


$$
g^{\prime}(\rho)=\left[V_{1}^{\prime}(\rho)^{2}+V_{2}(\rho)^{2}-2 V_{1}^{\prime} \cdot(\rho) V_{2}(\rho) \cdot \cos \gamma^{\prime}(\rho)\right]^{1 / 2},
$$

where $Y^{\prime}(\rho)$ is the angle between $V_{m}^{\prime}:(\rho)$ and $V_{\infty}(\rho)$. The change in relative kinetic energy caused by the ion-neutral collision is

$$
\frac{\mu}{2}\left(g^{2}-g^{\prime 2}\right)=\frac{\mu}{2}\left[V_{1}^{2}-V_{1^{\prime}}^{\prime 2}+2 V_{2}\left(V_{1}^{\prime} \cdot \cos \gamma^{\prime}-V_{1} \cos \gamma\right)\right]
$$

where $\mu$ is the reduced mass of the ion pair. From Eq. (G-3) we see that the.ion pair may lose relative kinetic energy by the transfer of leinetic energy to the neutral so that $V_{1}^{\prime} 2$ is less than $V_{1}^{2}$, or relative kinetic energy may be lost be decreasing the angle between $V_{m}$ and $V_{2}$ so that $V_{1}^{i} \cos \gamma^{\prime}>V_{1} \cos \gamma$. Since the relative kinetic energy at $r=\rho$ before the ion-neutral collision is given by

$$
T_{R}(\rho)=T_{R}(\infty)+e^{2} / \rho,
$$

in order for deactivation to occur, we must have

$$
\frac{\mu}{2}\left(g^{2}-g^{\prime 2}\right) \geq T_{R}(\infty)
$$

If we let $\Delta \mathrm{T}_{1}=\frac{\mathrm{M}_{1}}{2}\left(\mathrm{~V}_{1}^{2}-\mathrm{V}_{1}^{2}{ }^{2}\right)$, then for any given $\Delta \mathrm{T}_{1}$, deactivation occurs if

$$
\cos \gamma^{\prime} \geqslant \frac{T_{R}(\infty)-\left(\frac{M_{2}}{M_{1}+M_{2}}\right) \Delta T_{1}}{\mu V_{1}(\rho) V_{2}(\rho)\left(1-\frac{\Delta T_{1}}{T_{1}(\rho)}\right)^{1 / 2}}+\frac{\cos \gamma}{\left(1-\frac{\Delta T_{1}}{T_{1}(\rho)}\right)^{1 / 2}}
$$

where $M_{1}$ and $M_{2}$ are the masses of the two ions and $T_{1}(\rho)$ is the kinetic energy of ion one at $r=\rho$ before the ion-neutral collision.

In a consideration of the ion-neutral collision, it is helpful to use the vector diagram in Fig. 25(a) in which the vectors represent the asymptotic initial state; however, it is convenient to draw the vectors as if the ion and the neutral were just emerging from the scattering 

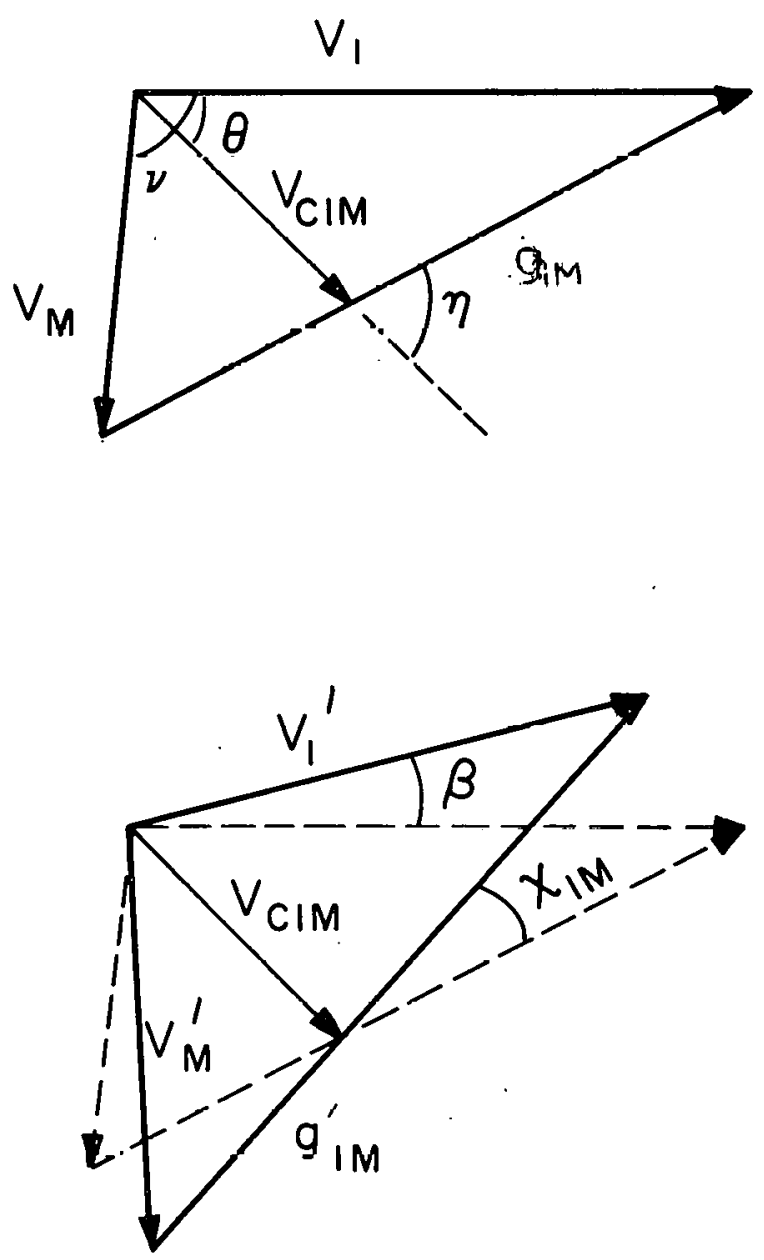

$M U-31889$

Fig. 25 (a). Vector diagram of the asymptotic initial state of an ion-neutral collision.

(b). Vector diagram of the asymptotic final state of the same ion-neutral collision showing the rotation of $\vec{g}_{1 \mathrm{M}}$ through the angle $x_{1 \mathrm{M}}$. 
center. If the ion-neutral collision.is elastic, then the collision will rotate the relative velocity vector of the ion-neutral, . $g_{m} 1 M$, through some angle $X_{1 M}$. This is illustrated in Fig. 25(b), where the dashed lines represent the initial state and the solid lines the asymptotic values after the collision. The angle of deflection of $V_{1}$ in the laboratory system is represented by $\beta$ in Fig $25\left(\right.$ b) and it can be found from ${ }^{44,45}$

$$
\tan (\beta+\theta)=\frac{\sin \left(x_{1 M}+\eta\right)}{x+\cos \left(x_{1 M}+\eta\right)},
$$

where $\eta$ is the angle between $\mathrm{V}_{m} \mathrm{C}_{1 \mathrm{M}}$ and $\mathrm{g}_{\mathrm{m}} \mathrm{M}$ before the collision; $\mathrm{V} C 1 \mathrm{M}$ is the ion-neutral center of mass velocity and is given by

$$
V_{m C 1 M}=\frac{M_{1} V_{1}+M_{M} V_{M}}{\left(M_{1}+M_{M}\right)},
$$

where $V_{m} M$ and $M_{M}$ are the velocity and mass of the neutral. The parameter $X$ in Eq. $(G-7)$ is defined as

$$
\mathrm{X}=\frac{\mathrm{M}_{1}+\mathrm{M}_{M}}{\mathrm{M}_{\mathrm{M}}} \frac{\mathrm{V}_{\mathrm{C} 1 \mathrm{M}}}{\mathrm{g}_{1 \mathrm{M}}}
$$

The angle $\theta$ between $\underset{m}{V_{1}}$ and $\underset{m}{V_{C 1 M}}$ can be found from

$$
\tan \theta=\frac{\sin \eta}{x+\cos \eta}
$$

where the angle $\eta$ is given by

$$
\cos \eta=\left[\frac{T_{1}(\rho)}{T_{R 1 M}}\left(\frac{M_{1}+M_{M}}{M_{M}}\right)-x^{2}-1\right] /(2 X)
$$

where $T_{R 1 M}=\frac{1}{2} \mu_{1 M} g_{1 M}{ }^{2}$ is the relative kinetic energy of the ionneutral system.

The amount of energy lost by ion one, $\Delta T_{1}$, for any deflection angle $x_{1 \mathrm{M}}$ is given by 


$$
\begin{aligned}
& \Delta T_{1}=M_{1}\left(V_{m 1}^{2}-V_{m 1}^{\prime 2}\right) / 2 \\
& =M_{1}\left[\left(V_{m} C_{1 M}+\frac{M_{M}}{\left(M_{1}+M_{M}\right)} g_{1 M}\right)^{2}-\left(V_{m}{ }_{1 M}+\frac{M_{M}}{\left(M_{1}+M_{M}\right)} g_{1^{\prime}}^{\prime} M\right)^{2}\right] / 2 \\
& =\frac{M_{1}{ }^{M} I_{M}}{\left(M_{1}+M_{M}\right)} V_{C 1 M} g_{1 M}\left[\cos \eta-\cos \left(\eta+x_{1 M}\right)\right] \\
& =2 X T_{R 1 M}\left(\frac{M_{M}}{M_{1}+M_{M}}\right)\left[\cos \eta\left(1-\cos x_{1 M}\right)\right. \\
& \left.+\sin \eta \sin x_{1 M}\right]
\end{aligned}
$$

The initial conditions $\eta$ and $T_{R 1 M}$ along with the ion-neutral force law. will determine $x_{1 \mathrm{M}}$ as a function of $\mathrm{b}_{1 \mathrm{M}}$, the ion-neutral impact parameter, and, as we saw above, if we know $\chi_{1 M}$ we can calculate $\Delta \mathrm{T}_{1}$ and $\beta$.

Now we can calculate $\gamma^{\prime}(\rho)$ if we know $\gamma(\rho), \beta$, and $\phi$, the angle between the plane of $\mathrm{V}_{2}, \mathrm{~V}_{1}$ and the plane of $\mathrm{V}_{2}, \mathrm{~V}_{1}^{\prime}$. Figure 26 (a) illustrates the angles, and from the geometry we find

$$
\cos \gamma^{\prime}(\rho)=\cos \beta \cos \gamma(\rho)+\sin \beta \sin \gamma(\rho) \cos \phi .
$$

Therefore, for any deflection $X_{1 M}$ we can calculate $\Delta \mathrm{T}_{1}$ and $\beta$, and from Eq. $(G-13)$ we can find the range of $\phi$ that results in deactivation; that is, the range that gives angles $\gamma^{\prime}(\rho)$ that satisfy the deactivation requirement in Eq. (G-6). We assume that all values of $\phi$ are equally probable, so the fraction of collisions with a given value of $\dot{\chi}_{1 \mathrm{M}}$ that causes deactivation is just the fraction of $2 \pi$ radians of the values of $\phi$ that give deactivation. Thus, if we know $V_{m}(\rho)$, $V_{m}(\rho)$, and $V_{m M}$ (as well as the masses), then the rate of deactivation for each $\chi_{1 M}$ is just the fraction of $\phi$ giving deactivation for that $\chi_{1 M}$ 

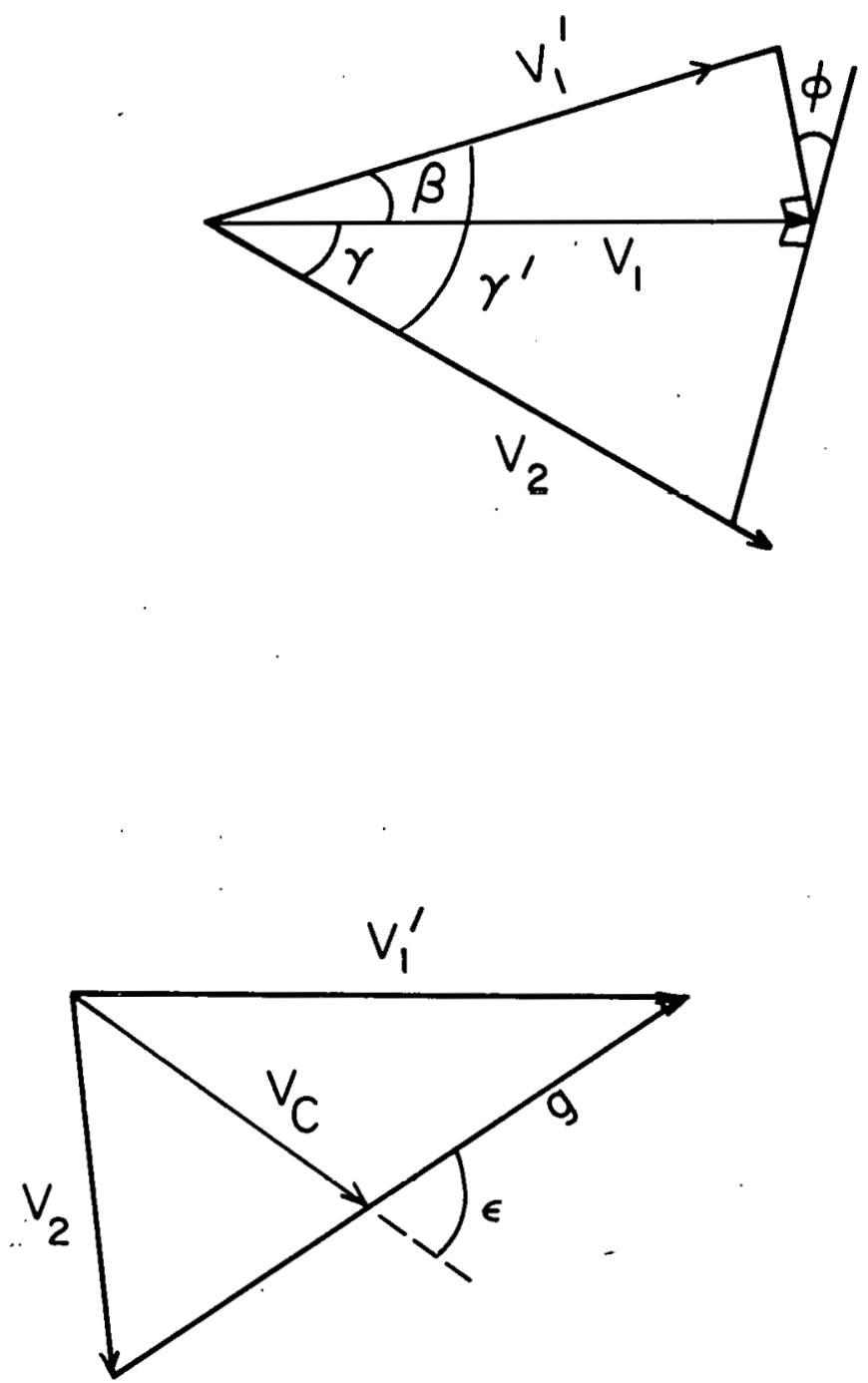

MU - 31890

Fig. $26(a)$. Diagram showing the angle $\phi$ between the plane of $\vec{V}_{2}, \vec{V}_{1}$ and the plane of $\vec{V}_{2}, \vec{V}_{1}^{\prime}$.

Fig. 26 (b). Vector diagram showing the angle $\epsilon$. 
times the rate of formation of that value of $x_{1 M} \cdot$ Figure 27 is a flow diagram of the calculation of $k_{3}$. The order of presentation is from the bottom of Fig. 27 to the top.

\section{Calculation of the Rate of Obtaining a Deflection $x_{1 \mathrm{M}}$}

The deflection angle $\chi_{1 \mathrm{M}}$ can be related to the impact parameter, $b_{1 M}$, of the ion-neutral collision, if the interaction potential is known. For the ion-induced dipole potential with a hard-sphere corc, Haooćc ${ }^{2 y}$ has showli tlial $x_{1 M}$ is a function of à pàrameter, $Y$, defined as

$$
Y=\left(\frac{\mu_{1 M}}{a_{M} e^{2}}\right)^{1 / 2} b_{1 M}{ }^{2} g_{1 M^{\prime}}
$$

where $\mu_{1 M}$ is the reduced mass of the ion-neutral system, $a_{M}$ is the polarizability of the neutral, and $e$ is the electronic charge. Also, the relation between $Y$ and $X_{1 M}$ depends upon the square root of the ratio of $\mathrm{T}_{\mathrm{R} .1 \mathrm{M}}\left(\mathrm{r}_{1 \mathrm{M}}-\infty\right)$ lo the depth of the potential well. ${ }^{29}$ 'I'his ratio is defined by $Z$, where

$$
Z=\left(\frac{H_{1 M}}{a_{M} e^{2}}\right)^{1 / 2} g_{1 M} s^{2},
$$

where $s$ is the radius of the hard-sphere corc. By using Hasséps equations, $X_{1 M}$ was calculated as a function of $Y$ and the results are shown in Fig. 28 for several values of $Z$. If we use $Z \leqslant 1$, then $X_{1 M}$ goes to $-\infty$ at $Y=2$, corresponding to an "'orbiting" collision.

Because the calculation was done numerically, it is convenient to calculate the rate of formation of a range of deflection angles, $\Delta X_{1 M}$. Alșo, since we can't tell whether we shall get a positive or a negative deflection for any given value of $b_{1 M}$ ', we want $\Delta\left|x_{1 M}\right|$. Further, since the observed scattering angle is between $-\pi$ and $+\pi$, we want $\Delta\left|\left(x_{1 M}-m 2 \pi\right)\right|$, where $m$ is a positive integer or zero, such that $-\pi \leqslant x_{1 M}-m 2 \pi \leqslant \pi$. Now, the rate of formation of deflections in the region $\Delta x_{1 M}$ can be taken as 
Calculation of $k_{3}$ for each value of $\gamma(\infty)$, $b$, and for collision with each ion of the ion pair

Assume values of:

This determines:

Assume a value of

This determines:

Assume values of:

This determines:

Eqs. $(G-8),(G-10)$, and $(G-11)$

Assume a value of:

This determines:

Eqs. $(G-6),(G-7)$, and $(G-12)$. b, $\gamma(\infty), \mathrm{T}_{1}(\infty), \mathrm{T}_{2}(\infty), \mathrm{M}_{1}, \mathrm{M}_{2}$

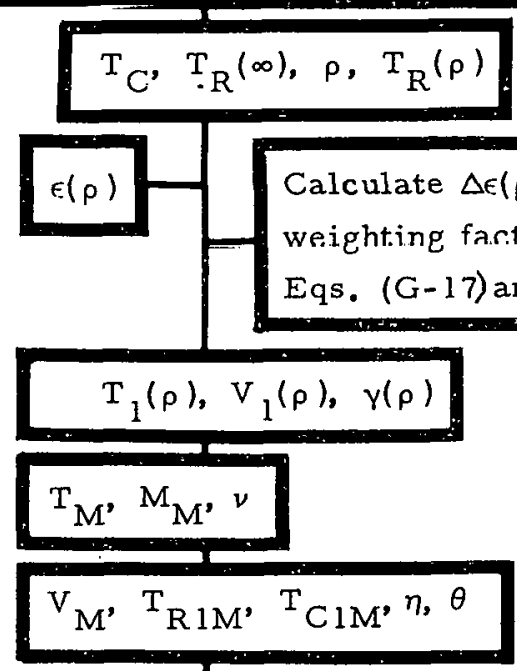

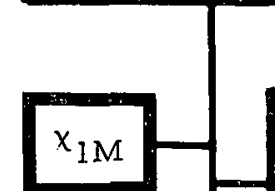

Calculate rate of formation of $\Delta x_{1 M}$ regions, $E q .(G-16)$.

$\beta, \Delta T_{1}, V_{1}^{\prime}(p)$, and the values of $\gamma(p)$ which cause deactivation it

Then find the fraction of $2 \pi$ radians of the angles $\phi$ which give values of $\gamma^{\prime}(\rho)$ which cause deactivation, Eq. $(G-13)$.

Fig. 27. Flow diagram of the calculation of $k_{3}$. 


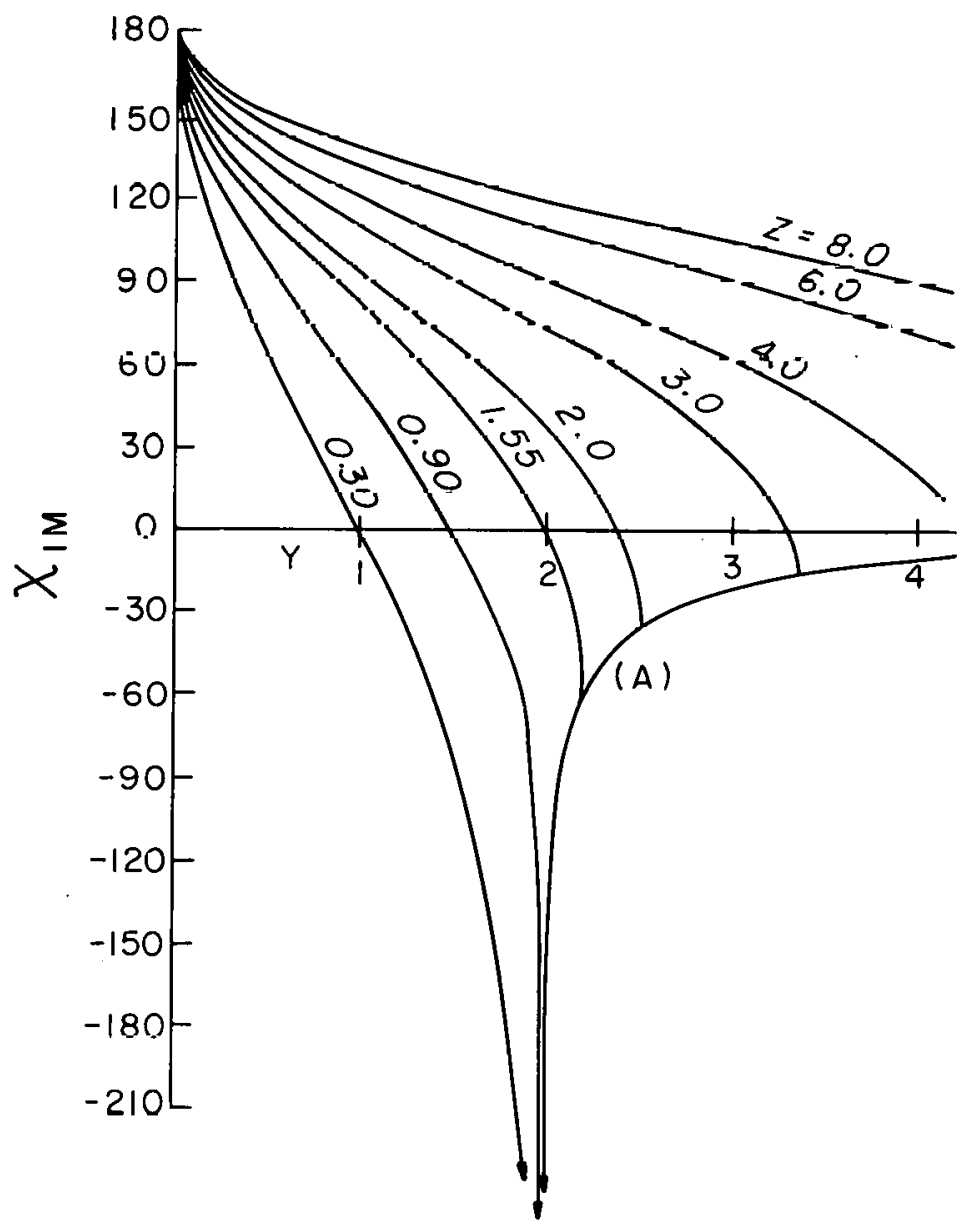

MU - 31091

Fig. 28. Deflection angle $X_{1 M}$ as a function of $Y$ for several values of $Z$. (A) Curve for pure ion-induced dipole potential; all finite values of $Z$ eventually connect with this curve. 


$$
\mathrm{d}\left(\Delta \mathrm{x}_{1 \mathrm{M}}\right) / \mathrm{dt}=\pi \Delta\left(\mathrm{b}_{1 \mathrm{M}}^{2} \mathrm{~g}_{1 \mathrm{M}}\right)[\mathrm{M}][\text { Ion] },
$$

where $[\mathrm{M}]$ and [Ion] are the neutral and ion concentrations, and

$\Delta\left(b_{1 M}^{2} g_{1 M}\right)=\left|\left(b_{1 M}^{2} g_{1 M}\right)_{1 M 2}-\left(b^{2} g_{1 M}\right) x_{1 M 1}\right|$.

where $\Delta x_{1 \mathrm{M}}=x_{1 \mathrm{M} 2}-x_{1 \mathrm{M} 1}$ and $\left(\mathrm{b}_{1 \mathrm{M}}^{2} g_{1 \mathrm{M}}\right) x_{1 \mathrm{M} 2}$

is the value of $b_{1 . M}^{2} g_{1 M}$ that gives the deflection $x_{1 M 2}$.

Then, the rate of formation of deflections in the region $\Delta \mid\left(x_{1 M}-\mathrm{m} 2 \pi\right)$

is the sum of terms similar to those in the right side of Eq. (G-16) for all the different values of $\mathrm{b}_{1 \mathrm{M}}^{2} \mathrm{~g}_{1 \mathrm{M}}$ that give deflections in the region $\Delta\left|\left(x_{1 M}-m 2 \pi\right)\right|$. Appendix $B$ gives the values of $\Delta \mathrm{Y}$ for various $\Delta\left|\left(X_{1 M}-m 2 \pi\right)\right|$ regions for several values of $Z$. By knowing the reduced mass and the polarizability, we can calculate the rate constant for formation of deflection angles in a region $\Delta\left|\left(X_{1 M}-m 2 \pi\right)\right|$.

D. Calculation of the Rate of Collisional Deactivation

In Sec. VII-B we saw that by knowing $V_{m}(\rho), V_{2}(\rho), V_{m M}$, and the masses, we could calculate the rate of deactivation if we.knew the rate of formation of $X_{1 M}$, and we saw how to calculate this in Sec. VII-C. We can calculate the fraction of collisions with $+X_{1 M}$ that give deactivation, average this with the fraction giving deactivation with $-X_{1 M}$, and then weight this average fraction by the rate of formation of $X_{1 M}$ in the region $\Delta\left|\left(x_{1 M}-m 2 \pi\right)\right|$. This is repeated until the range of $X_{1 M}$ from 2 deg to $180 \mathrm{deg}$ has been covered, and the sum gives the total rate of deactivation. (The 2 deg lower limit is used because the cross section goes to infinity as $x_{1 M}$ goes to zero. The arbitrary cutoff at 2 deg is rather unimportant, since the results of the calculation indicate that deflections of 20 deg or less are likely to cause deactivation only for the cases with small values of $\rho$ )

If we know $V_{1}(\rho)$ and $V_{m}(\rho)$ we can calculate the rate of deactivation by averaging over the possible values of $\mathrm{VmM}_{\mathrm{M}}$. We determine the magnitude of $\dot{\mathrm{V}}_{M}$ by assuming that the neutral always has a kinetic energy of $(3 / 2) k_{B} T$ when $r_{1 M}=\infty$. Then we assume that all angles 
between $V_{m}$ and $V_{m}$ are equally probable at $r_{1 M}=\infty$, and average the rate of deactivation over all values of $v$, where $v$ is the angle between $\underset{m M}{V_{M}}$ and $V_{m}$ at... $r_{1 M}=\infty$.

Next, we want to average over the possible values of $\mathrm{V}_{1}(p)$ and $\mathrm{V}_{m}(\rho)$. These vectors are determincd through knowledge of the kinetic energy of the motion of the center of mass of the ion pair ${ }^{T}{ }_{C}$, the value of $T_{R}(\rho)$, the angle $\epsilon$ between $g$ and the velocity of the center of mass $\vec{V}_{m}$, and the masses $M_{1}$ and $M_{2}$. Figure 26(b) illustrates the vector relationohp. Hcre again the rate of deactivation is caliulated for each value of $\epsilon$ and these rates are weighted by the proba:bility of having $\epsilon$ in the region $\Delta \epsilon$. However, the angle $\epsilon$ does not have a uniform distribution; instead, the value of $\epsilon(\rho)$ is related to $\epsilon(r=\infty)$, to the value of $\rho$, and to the value of $b$, the ion-ion impact parameter. The reason for this is that the angle $\Delta \epsilon$ between $\epsilon(\infty)$ and $\epsilon(p)$ is just the deflection angle of $g$ in the center-of-mass coordinates and the magnitude of this deflection depends on how nearly the collision is a headi-on collision, and on the magnitude of $\rho$. At $\rho=\infty$ we than have $\Delta \epsilon=0$ or $\Delta \epsilon=\chi_{12}$, where $\chi_{12}$ is the deflection angle of $g$, depending on whether $p=\infty$ is on the incoming or on the outgoing leg of the ion pair collision. The relationship between $\epsilon(\rho)$ and $\epsilon(\infty)$ on the incoming leg is given by

$$
\epsilon(\rho)=\epsilon(\infty) \pm\left(\delta_{0}-\delta\right),
$$

where $\therefore \quad \tan _{0}=1 /\left(e_{C}^{2}-1\right)^{1 / 2}$

and $\quad \tan \delta=\tanh \xi /\left(\mathrm{e}_{\mathrm{C}}^{2}-1\right)^{1 / 2}$,

with ${ }^{e} \mathrm{C}$ being the eccentricity of the orbit; ${ }^{e_{C}}$ is given by 46

$$
e_{C}=\left(1+4 T_{R}^{2}(\infty) b^{2} / e^{4}\right)^{1 / 2}
$$

where $e$ is the electronic charge. The parameter $\xi$ can be found by using $r=\rho$ in the relationship 47

$$
\mathrm{r}=\mathrm{a}\left(\mathrm{e}_{\mathrm{C}} \cosh \xi-1\right)
$$


whe re

$$
\left.a=e^{2} / 2 T_{R}(\infty)\right]
$$

On the outgoing leg the relation between $\epsilon(\rho)$ and $\dot{\epsilon}(\infty)$ [where $\epsilon(\infty)$ refersi; to $r=\infty$ before the ion collision]. is

$$
\begin{aligned}
\epsilon(\rho) & =\epsilon(\infty) \pm 2 \delta_{0} \mp\left(\delta_{0}=\delta\right) \\
& =\epsilon(\infty) \pm\left(\delta_{0}+\delta\right) .
\end{aligned}
$$

The factor $2 \delta_{0}$ comes from the relation $x_{12}=2 \delta_{0}$.

We calculate the welghting factors for the $\Delta \epsilon$ regions by finding the distribution of $\epsilon$ corresponding to equal values of $\Delta b^{2}$ from $b^{2}=0$ to $b^{2}=B^{2}$, where $B$ is the impact parameter that gives $\rho$ as the distance of closest approach. This distribution is then used to calculate the weighting factors for the $\Delta \epsilon$ regions. This procedure does introduce some error, because the concentration at $r=\rho$ on the outgoing leg is smaller than the concentration on the incoming leg, and is also a function of the impact parameter; some of the ions recombine at separations smaller than $\rho$ and the ions with small impact parameters are more likely to recombine. However, if the total fraction of ions recombining is fairly small, this procedure should not cause a. very large error.

From the above discussion we see that by knowing $T_{R}(\infty), T_{C}$, and $\rho$ (and the masses), we can calculate the rate of deactivation. Now, $T_{R}(\infty), T_{C}$, and $\rho$ are all determined by the values of the ion kinetic energies ar $r=\infty, T_{1}(\infty)$ and $T_{2}(\infty)$, by the impact parameter, $b$, and by the angle, $\gamma(\infty)$, between $\mathrm{V}_{1}(\infty)$ and $\mathrm{V}_{2}(\infty)$. In this calculation we took $T_{1}(\infty)=T_{2}(\infty)=(3 / 2) k_{B} T$. Therefore, we can find the rate constant for deactivation for any given values of $b$ and $\gamma(\infty)$, and we are now ready to see how actually calculate the recombination coefficient. 
E. Calculation of the Recombination Coefficient, $a_{T}$

If we specify the states of the ion pair as being given by regions $b_{i}^{2}+\Delta b^{2}$ and by the value of $\gamma(\infty)$, then for each $\gamma(\infty)$ we shall have a series of distances $r_{C}\left[b_{i}, \gamma(\infty)\right]$ which are the distances of closest approach corresponding to the impact parameters, $b_{i}$, where

$$
b_{i}^{2}=r_{C}^{2}\left[b_{i}, \gamma(\infty)\right]\left[1+e^{2} / r_{C}\left[b_{i}, \gamma(\infty)\right] T_{R}[\gamma(\infty)]\right] \text {. }
$$

'l he total, rate at which ion pairs go through ${ }^{\mathrm{C}_{\mathrm{C}}}\left[\mathrm{b}_{\mathrm{m}}, \gamma(\infty)\right]$ on the incoming leg is given by $\pi b_{I X 1}^{2} g[\gamma(\infty)]\left[A^{+}\right]\left[B^{-}\right]$, where

$$
g[\gamma(\dot{\nu})]-\left\{2 \mathrm{~T}_{\mathrm{R}}[\gamma(w)]^{\prime} \mu\right\}^{1 / 2},
$$

and where we have assumed that no ion recombination has taken place at distances greater than ${ }_{C_{C}}\left[b_{m}, \gamma(\infty)\right]$. The rate at which undeactivated ion pairs arrive at the next inner shell at ${ }{ }_{C}\left[b_{m-1}, \gamma(\infty)\right]$ on the incoming leg is $\pi b_{m-1}^{2} g[\gamma(\infty)]\left[\mathrm{A}^{+}\right]\left[\mathrm{B}^{-}\right]$minus the number of ion pairs with impact parameters less than $b_{m-1}$ that have already been deactivated between ${ }_{{ }_{C}}\left[b_{m}, \gamma(\infty)\right]$ and $r_{C}\left[b_{m-1}, \gamma(\infty)\right]$. Similarly, the rate of formation of unreacted ion pairs whose distance of closest approach is between $r_{C}\left[b_{i}, r(m)\right]$ and $r_{C}\left[b_{i+1}, r(m)\right]$ (those ion paire will be $r e-$ forred to as ion pairs in the ith state) is given by $P_{i}$, where

$$
P_{i}=\pi\left(b_{i+1}^{2}-b_{i}^{2}\right) g[\gamma(\infty)]\left(1-F_{i}\right)\left[A^{+}\right]\left[B^{-}\right] \text {. }
$$

Here, $F_{i}$ is the fraction of ion pairs originally in the ith state which have been deactivated in outer shells; $F_{i}$ is given by

$$
F_{i}=\sum_{j=i+1}^{m-1} f(i, j),
$$

where $f(i, j)$ represents the fraction of ion pairs in the ith state that are deactivated in the $\mathrm{jth}$ state; $f(i, j)$ is given by 


$$
f(i, j)=\frac{k_{3}(j)[M]}{\langle 1 / \tau(i,-j)\rangle+k_{3}(j)[M]}\left[1-\sum_{q=j+1}^{m-1} f(i, q)\right] .
$$

Here, $k_{3}(j)$ is the average rate constant for deactivation by a collision of the positive ion in the region between $r_{C}\left[b_{j}, \gamma(\infty)\right]$ and $r_{C}\left[b_{j+1}, \gamma(\infty)\right]$ plus the average rate constant for deactivation by a collision of the negative ion in the same region, [M] is the neutral concentration, and $\langle 1 / \tau(i, j)\rangle$ is the average of the reciprocal of the time required for ions in the ith state to go from $r_{C}\left[b_{j+1}, \gamma(\infty)\right]$ to $r_{C}\left[b_{j}, \gamma(\infty)\right]$. The time $\tau(i, j)$ can be calculated from Eq. $(G-21)$, which relates $r$ to $\xi$ and ${ }^{e}{ }_{C}$, and from the relation ${ }^{47}$ between the time $t, e_{C}$, and $\xi$. We have

$$
t=\left(\mu a^{3} / e^{2}\right)^{1 / 2}\left(e_{C} \sinh \xi-\xi\right)
$$

where $e_{C}$ and a are defined in Eqs. $(G-20)$ and $(G-22)$. Since $e_{C}$ is a function of the impact parameter, the $\tau(i, j)$ values are different for the different values of $\underline{i}$.

The fraction of ion pairs in the ith state that are deactivated between ${ } C^{\left[b_{i+1}, \gamma(\infty)\right]}$ and ${ } C^{\left[b_{i}, \gamma(\infty)\right]}$ is given by $G_{i}$, where

$$
G_{i}=\frac{k_{3}(i):[M]\left(1-F_{i}\right)}{\langle 1 / \tau(i, i:)\rangle+k_{3}(i)[M]},
$$

where $\langle 1 / \tau(i, i)\rangle$ is the average of the reciprocal of the time that the ion pairs in the ith state spend between the time when they reach ${ }^{r_{C}}\left[b_{i+1}, \gamma(\infty)\right]$ on the incoming leg and the time when they reach ${ }^{r}{ }^{\left[b_{i+1}, \gamma(\infty)\right]}$ on the outgoing leg.

The fraction of ion pairs in the ith state deactivated between $r_{C}\left[b_{j}, \gamma(\infty)\right]$ and $r_{C}\left[b_{j+1}, \gamma(\infty)\right]$ on the outgoing leg is $h(i, j)$ : We have

$$
h(i, j)=\frac{k_{3}(j)[M][1-H(i, j)]}{\langle 1 / \tau(i, j)\rangle+k_{3}(j)[M]},
$$


where $1 / \tau(i, j)$ is the same as in Eq. $(G-27)$ and $[1-H(i, j)]$ represents the ion pairs initially in the ith state that are still not deactivated by the time they reach ${ }^{C_{C}}\left[b_{j}, \gamma(\infty)\right]$ on the outgoing leg, and $H(i, j)$ is given by

$$
H(i, j)=\sum_{q=i}^{j-1} h(i, q),
$$

with

$$
h(i, i)=E_{i}+G_{i}
$$

'l'he total rate of deactivation of ion pairs in the $\underline{\text { tth }}$ state is $D_{i}$, where

$$
D_{i}=I I(i, \cdots) \pi\left(b_{i+1}^{2}-b_{i}^{2}\right) g[\gamma(00)]\left[A^{+}\right]\left[B^{-}\right],
$$

where $[1-\mathrm{H}(\mathrm{i}, \mathrm{m})]$ is the undeactivated fraction of ion pairs in the ith state that passes through ${ }_{{ }_{C}}\left[b_{m}, \gamma(\infty)\right]$ on the outgoing leg. Therefore, the total rate of formation of deactivated ion pairs $\left(\mathrm{A}^{+} \mathrm{B}^{-}\right)$is

$$
\begin{aligned}
d\left[\left(A^{+} B^{-}\right)\right] / d t= & \sum_{i=0}^{m-1} D_{i}=\pi g[\gamma(\infty)]\left[A^{+}\right]\left[B^{-}\right] \\
& \sum_{i=0}^{m-1} H(i, m)\left(b_{i+1}-b_{i}\right)^{2}
\end{aligned}
$$

Then, by assuming that the three-body recombination coefficient, ${ }^{a} \mathrm{~T}$, is the same as the rate constant for formation of deactivated ion pairs, we have

$$
a_{T}\left[b_{m}, \gamma(\infty)\right]=1 /\left[A^{+}\right]\left[B^{-}\right] \sum_{i=0}^{m-1} D_{i} \text {. }
$$

We can now compute an average value of $a_{T}\left(b_{m}\right)$ by averaging over $\gamma(\infty)$, assuming that $\gamma(\infty)$ has a uniform distribution. 
Ideally, the values of $\mathrm{k}_{3}$ (i) would go to zero fast enough so that $a_{T}\left(b_{m}\right)$ would converge to a limit where the use of larger values of $b_{m}$ would not change $a_{T}$. However, because of the $b_{m}^{2}$ terms in $a_{T}$, the values of $k_{3}$ have to fall off faster than $1 / b^{2}$, and this doesn't seem to be the case when Eq. $(G-1)$ is used as a requirement for deactivation. The divergence of a seems to be a result of the long-range nature of the Coulomb force. We can still estimate relative third-body efficiencies by calculating $a_{T}\left(b_{m}\right)$ for various values of $b_{m}$ to: give values of $a_{T}$ that are comparable to the experimental values and taking ratios lu get relative efflciencies.

Another consideration is that in the foregoing discussion we assumed that once an ion pair was deactivated, it would inevitably undergo charge neutralization. This assumption is somewhat in error, since the ion pair may gain enough relative kinetic energy in a later collision to allow its ions to separate to infinity. Also, since the chance of charge neutralization probably depends very strongly on the distance of closest approach, ${ }^{r} \mathrm{C}$, the deactivated ion pairs with large values of ${ }^{r} \mathrm{C}$ may take a very long time to undergo charge neutralization; therefore, they have more opportunity to gain relative kinetic energy from another collision. (This effect is enhanced by the fact that the ion pairs with lower relative kinetic energy have smaller values of minimum and maximum separations if the eccentricity is the same; also, the ion pairs with lower relative kinetic energy are less likely to gain enough relative kinetic energy to be able to dissociate.) Thus, it may be that the rate of recombination is not the total rate of formation of deactivated ion pairs but only the rate of formation of deactivated ion pairs that have ${ }{ }_{C}$ 's below some maximum value. In our calculation we also tried using the requirement for deactivation that the ion pair lose enough relative kinetic energy so that

$$
T_{R}^{\prime}(\rho) \leqslant\left(e^{2} / \rho\right)-E_{D} ;
$$

where $E_{D}$ is the extra amount of energy that must be lost. The idea is that if the ion pair has a relative kinetic energy which is, say, $0.5 \mathrm{k}_{\mathrm{B}} \mathrm{T}$ or $\mathrm{k}_{\mathrm{B}} \mathrm{T}$ below the negative of the potential energy, then a 
further ion-neutral collision is less likely to enable the ion pair to dissociate. When we had $E_{D}=k_{B}$, then no deactivation occurred for ion pairs whose impact parameters were greater than $1500 \AA$, so that $a_{T}\left(b_{m}\right)$ is independent of $b_{m}$ for $b_{m} \geqslant 1500 \AA$.

Appendix C contains the FORTRAN-II program for the calculation of the specific rate of recombination.

\section{F. Rcoulto of the Dotailod Calculation}

A general result of the calculation is that the amount of relative kinetic energy that must be lost to cause deactivation is quite important in determining the rate constant $k_{3}$ for the deactivation reaction. Figure 29 shows a plot of $k_{3}$ vs $p$, the lon-iun separaliun al llet line of the deactivating collision, for argon atoms deactivating $\mathrm{NO}^{+}, \mathrm{NO}_{2}^{-}$ ion pairs by collision with the $\mathrm{NO}^{+}$: The solid lines give the values when the $T_{R}(\infty)=0.23 k_{B} T\left[\gamma(\infty)=30^{\circ}\right], T_{R}(\infty)=1.5 k_{B} T\left[\gamma(\infty)=90^{\circ}\right]$, and $T_{R}(\infty)=2.77 \mathrm{k}_{B} \mathrm{~T}\left[\gamma(\infty)=150^{\circ}\right]$ and when the requirement for deactivation is $E_{D}=0$ in Eq. $(G-36)$. The dashed lines give the results for the same values of $T_{R}(\infty)$ when $E_{D}=0.5 k_{B} T$ is the requirement for deactivation. From this graph we see that the smaller the relative kinetic energy that must be lost, the greater the value of $k_{3}$.

There is a factor that reduces the contribution to the anverage value of a from the ion pairs having small values of $T_{R}(\infty)$, and this is the smaller rate of formation of ion pairs at a given value of $\rho$, which is caused by the smaller value of $g(\infty)$. However, some of this reduction is canceled because the lower value of $g(\infty)$ lowers the value of $\mathrm{g}$ at all ion separations, so that the ion pairs with smaller values of $g(\infty)$ spend a longer time in the region where the ion-ion separation is small. Thus, these ion pairs have an increased chance to collide with a neutral while inside the critical region. The increase in, $\mathrm{k}_{3}$ for the smaller values of $T_{R}(\infty)$ is generally large enough so that these values of $T_{R}(\infty)$ make the largest contribution to the average value of $a$. The ratios of $\mathrm{k}_{3}$ 's for the heavy gases to $\mathrm{k}_{3}$ 's for the light gases are smaller for the smaller values of $T_{R}(\infty)$. This is because the light neutrals can remove only a small fraction of the ion's energy and 


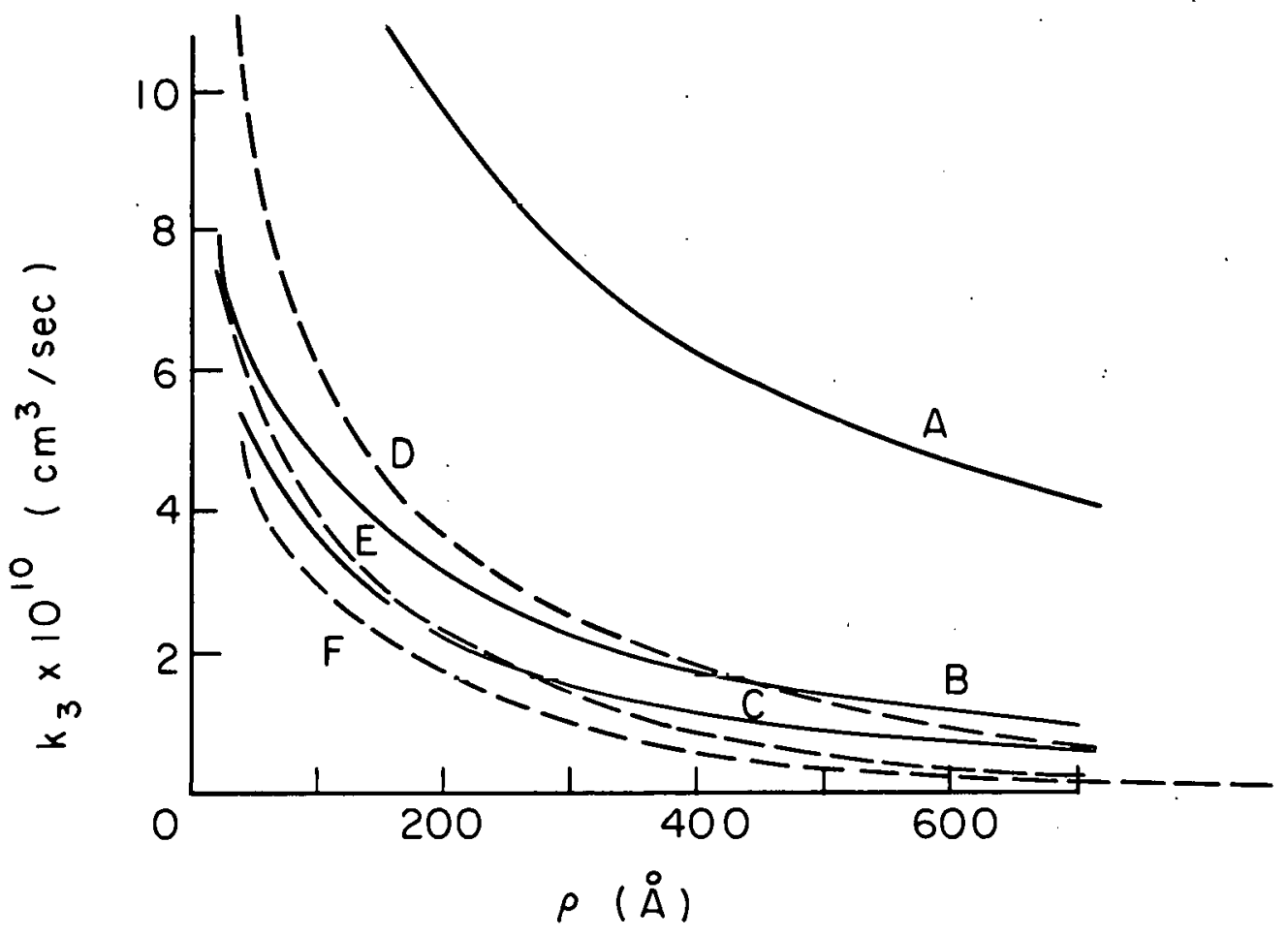

$M U-31892$

Fig. 29. Plot of $k_{3}$ vs $\rho$ for $\mathrm{NO}^{+}-$Ar collisions. 
cannot cause large deflections of $\mathrm{V}_{1}(\rho)$, so that the light neutrals are not able to deactivate very well when large amounts of $T_{R}$ must be lost.

It is intcresting to notice that if the scattering of ion one is influenced by the presence of ion two so that scattering along the direction of $r$ is favored, then it is more difficult for deactivation to take place. This is because the focusing tends to favor situations, in which $\gamma^{\prime}(p)$ is a large angle, so that ion one has to lose a greater amount of energy before deactivation can occur. However, the focusing decreases the distance of closest approach, so the purely bimolecular charge neutralization process is more important.

Another general feature of the results is that when the neutral is heavier than the ion, the fact that the ion can transfer only a fraction of its energy in a collision is compensated for somewhat because large deflections of $V_{1}(\rho)$ are possible, so that it is possible to make $\gamma^{\prime}(\rho)$ a small angle. For example, one might expect that the rather large difference in masses between $\mathrm{NO}_{2}^{-}$and $\mathrm{Xe}$ would reduce the thirdbody efficiency of $\mathrm{Xe}$ when compared with $\mathrm{Ar}$, since the maximum possible energy transfer is less when $\mathrm{NO}_{2}^{-}$collides with a xenon atom than when it collides with an argon atom. However, the increased probability of large deflections of $V_{1}(\rho)$ makes the chances better that $\gamma^{\prime}(\rho)$ will be small, and this more than compensates for the decrease in the maximum possible energy transfer.

We made calculations of a fur lle deactivation of $\mathrm{NO}^{+}, \mathrm{NO}_{2}^{-}$; and $\mathrm{NU}\left(\mathrm{NO}_{2}\right)^{+},\left(\mathrm{NO}_{2}\right)_{2}^{-}$ion pars by each of the neulrals sludied. The calculations were made for the cases in which the requirement for deactivation in Eq. $(G-36)$ is $E_{D}=0, E_{D}=0.5 k_{B} T$, and $E_{D}=k_{B} T$. The step sizes used in carrying out the calculations were such that there is an estimated uncertainty of 10 to $25 \%$ in the predicted values of $a$.

To approximate the effect of the purc bimolecular recombination process in our calculation, we considered that the $\mathrm{NO}^{+}, \mathrm{NO}_{2}^{-}$ion pairs always underwent charge neutralization when thcy reached the ${ }^{r} \mathrm{C}$ corresponding to $\mathrm{b}=100 \AA$ for each value of $\gamma(\infty)$. This procedure gave a predicted $k_{0}$ of $1.8 \times 10^{-7} \mathrm{~cm}^{3} / \mathrm{sec}$ when there was no third-body 
deactivation of the ion pairs in the outer regions. This same $k_{0}$ was also used for the $\mathrm{NO}\left(\mathrm{NO}_{2}\right)^{+},\left(\mathrm{NO}_{2}\right)_{2}^{-}$ion pair. We found that when there was third-body deactivation in which $a_{T}$ was. less than $2 \times 10^{-6} \mathrm{~cm}^{3} / \mathrm{sec}$, then the decrease in $k_{0}$ was less than $0.6 \times 10^{-7} \mathrm{~cm}^{3} / \mathrm{sec}$. So, the assumption $a=k_{0}+a_{T}$, with $k_{0}$ equal to the zero pressure limit of $a$, seems to be fairly good for these experiments.

Table XVI gives the values of $a_{T}$ calculated for helium at a pressure of 10 torr for the two different ion pairs and the three values of $E_{D}$. The low polarizability of helium makes the predicted values of a depend upon the choice of the hard-sphere radius, $s$, and Table XVI gives the results using the values of s listed in Table IV of Sec. IV. In the other gases the predicted values of a are only slightly dependent on the choice of $s$. The value of a depends strongly on the choice of the maximum impact parameter, $b_{m}$. For $E_{D}=k_{B} T$, there is no increase in $a$ when $b_{m}$ increases beyond $1500 \AA$, but for the other values of $E_{D^{\prime}}$ a will still increase when larger values of $b_{m}$ are used.

To have the prodicted values of $u$ be as large as the observed values, we have to use values of $b_{m}$ that are lárger than the values of $b_{1}$ [the maximum impact parameter to give $\left(\mathrm{A}^{+} \mathrm{B}^{-}\right)^{*}$ ] determined ex perimentally from the high-pressure limit of $a$. However, in the experimental determination of $k_{1}$, we assumed that every ion pair with an impact parameter less than $b_{1}$ could be deactivated, and here we are assuming that no ion pair whose impact parameter $\mathrm{is}$ greater than $\mathrm{b}_{\mathrm{m}}$ is deactivated. Therefore, we might expect $\mathrm{b}_{\mathrm{m}}$ to be larger than $b_{1}$. Furthermore, because $b_{m}$ is larger than $b_{1}$, the values of $k_{3}$ that we estimated from the experimental value of $b_{1}$ are larger than the calculated values of $k_{3}$.

Since the ions actually present during the experiments were probably. mixtures of clustered and unclustered ions, the best prediction of the relative efficiencies probably is some value between the predicted values for $\mathrm{NO}^{+}, \mathrm{NO}_{2}^{-}$ion pairs and the values for the $\mathrm{NO}\left(\mathrm{NO}_{2}\right)^{+},\left(\mathrm{NO}_{2}\right)_{2}^{-}$ions chosen as examples of possible ion clusters. Also, since the predictions in Sec. VI. A indicated that xenon and krypton 
Table XVI. Predicted values of $a_{T}$ in NO-He mixtures at a total pressure of 10 torr. ${ }^{a}$

\begin{tabular}{|c|c|c|c|c|c|c|}
\hline \multirow{2}{*}{$b_{m}^{b}$} & \multicolumn{2}{|c|}{$E_{D}=0$} & \multicolumn{2}{|c|}{$E_{D}=0.5 k_{B} T$} & \multicolumn{2}{|c|}{$\mathrm{E}_{\mathrm{D}}=\mathrm{k}_{\mathrm{B}} \mathrm{T}$} \\
\hline & $\mathrm{G}^{\mathrm{C}}$ & $L-J^{d}$ & $\mathrm{G}$ & $\overline{L-J}$ & G & L-J \\
\hline \multicolumn{7}{|c|}{$\mathrm{NO}^{+}, \mathrm{NO}_{2}^{-}$ion pair } \\
\hline 400 & 0.40 & 0.48 & 0.20 & 0.24 & & \\
\hline 600 & 1.22 & 1.41 & 0.49 & 0.58 & 0.27 & 0.33 \\
\hline 800 & 2.60 & 2.92 & 0.95 & 1.13 & 0.49 & 0.60 \\
\hline 1000 & 4.81 & 5.34 & 1.60 & 1.93 & 0.69 & 0.84 \\
\hline 1200 & 8.3 & 9.1 & 2.39 & 2.90 & $0 . \overline{7} \overline{7}$ & 0.94 \\
\hline 1500 & 16.5 & 18.0 & 3.62 & 4.43 & 0.79 & 0.97 \\
\hline \multicolumn{7}{|c|}{$\mathrm{NO}\left(\mathrm{NO}_{2}\right)^{+},\left(\mathrm{NO}_{2}\right)_{2}^{-}$Ion Pair } \\
\hline 400 & 0.15 & 0.20 & 0.05 & 0.08 & & \\
\hline 600 & 0.52 & 0.62 & 0.19 & 0.27 & 0.10 & 0.16 \\
\hline 800 & 1.27 & 1.50 & 0.44 & 0.65 & 0.16 & 0.26 \\
\hline 1000 & 2.75 & 3.24 & 0.74 & 1.13 & 0.19 & 0.31 \\
\hline 1200 & 5.18 & 6.22 & 0.99 & 1.54 & 0.19 & 0.31 \\
\hline 1500 & & & 1.24 & 1.96 & 0.19 & 0.31 \\
\hline
\end{tabular}

${ }^{a}$ Numbers are $a_{T} \times 10^{8} \mathrm{~cm}^{3} / \mathrm{sec}$.

b Maximum value of impact parameter.: for deactivation.

${ }^{c}$ Values of $a_{T}$ when $s$ was estimated from the Goldschmidt Table (reference 31 ).

${ }^{d}$ Values of $a_{T}$ when $s$ was estimated from Lennard-Jones $\sigma$ values (reference 32 ). 
were the neutrals most likely to take part in the formation of ion clusters, we calculated the relative efficiencies expected if $\mathrm{NO}(\mathrm{Xe})^{+}$ and $\left(\mathrm{NO}_{2}\right)_{2}^{-}$were the ions in the $\mathrm{NO}-\mathrm{Xe}$ mixtures and if $\mathrm{NO}(\mathrm{Kr})^{+}$and $\left(\mathrm{NO}_{2}\right)_{2}^{-}$were the ions in the $\mathrm{NO}-\mathrm{Kr}$ mixtures. The relative efficiencies for the $\mathrm{NO}(\mathrm{M})^{+},\left(\mathrm{NO}_{2}\right)_{2}^{-}$ions were calculated as relative to $\mathrm{NO}\left(\mathrm{NO}_{2}\right)^{+}$ and $\left(\mathrm{NO}_{2}\right)_{2}^{-}$being the ions in the NO-He mixtures.

Table XVII gives the relative third-body efficiencies of the various neutrals, relative to helium as the third-body gas. The values of the relative efficiencies were computed by taking the ratios of $a_{T}$ calculated for a pressure of 10 lör in tacli lieutial gas.

The numerical values of the relative efficiencies depend somewhat on the choice of $\mathrm{b}_{\mathrm{m}}$. The predicted order of relative efficiency is $\mathrm{Xe}>\mathrm{Kr}>\mathrm{N}_{2} \approx \mathrm{Ar}>\mathrm{D}_{2} \approx \mathrm{H}_{2}>\mathrm{He}$. The relative efficiency of $\mathrm{N}_{2}$ is predicted to be less than $\mathrm{Kr}$, where, experimentally it seems to be greater than $\mathrm{Kr}$. In Sec. IV. C we saw that the ion-neutral interaction potential used here predicted the ionic mobilities in $\mathrm{N}_{2}$ to be $10 \%$ higher than the experimental values, so we might expect that the predicted values of $a_{T}$ in $N_{2}$ might be about $10 \%$ low. Also, the diatomic neutral molecules do not have the spherical symmetry that we have assumed, and besides that, the diatomic molecules may remove some of the ion's energy by exciting the rotational or vibrational levels. Therefore we might expect our predictions for $\mathrm{H}_{2}, \mathrm{D}_{2}$, and $\mathrm{N}_{2}$ to be somewhat in error.

Table XVIII gives the predicted relative efficiencies when $E_{D}=0.5 k_{B} T$ is the requirement for deactivation, and Table XIX gives the predictions when $E_{D}=k_{B} T$. In these two tables the predicted relative efficiencies are in reasonable agreement with the experimental results if the ions are $\mathrm{NO}^{+}$and $\mathrm{NO}_{2}^{-}$. However, if the ions are clustered, then the predicted values of $a$ in the light gases $\left(\mathrm{H}_{2}, \mathrm{D}_{2}\right.$, and $\left.\mathrm{He}\right)$ are rather low, so that the predicted relative efficiencies of the heavy gases are considerably above the observed values..

The values of a were calculated for pressures up to 500 torr in order to investigate the predicted pressure dependence. The predictions at the higher pressures are less reliable than the predictions at 
Table XVII. Relative third-body efficiencies when $E_{D}=0$

\begin{tabular}{|c|c|c|c|c|c|c|c|c|c|c|c|}
\hline \multirow{2}{*}{$\begin{array}{l}b_{m}^{a} \\
(\AA)\end{array}$} & \multirow{2}{*}{$\frac{\mathrm{NO}^{+}}{\mathrm{G}^{\mathrm{b}}}$} & \multirow{2}{*}{$\frac{\mathrm{NO}_{2}{ }^{-}}{\mathrm{L}-\mathrm{J}^{\mathrm{C}}}$} & $\mathrm{N}_{2} \mathrm{O}_{3}$ & ${ }_{2} \mathrm{O}_{4}^{-}$ & \multicolumn{2}{|c|}{$\mathrm{NO}^{+}, \mathrm{NO}_{2}^{-}$} & \multicolumn{3}{|c|}{$\mathrm{N}_{2} \mathrm{O}_{3}^{+}, \mathrm{N}_{2} \mathrm{O}_{4}^{-}$} & \multicolumn{2}{|c|}{$\mathrm{NO}(\mathrm{M})^{+}, \quad \mathrm{N}_{2} \mathrm{O}_{4}^{-}$} \\
\hline & & & $\mathrm{G}$ & $\mathrm{L}-\mathrm{J}$ & $\underline{G}$ & $\underline{L-J}$ & $G$ & L-J & & $\mathrm{G}^{\mathrm{d}}$ & $L-J^{d}$ \\
\hline \multicolumn{5}{|c|}{ Nitrogen } & \multicolumn{4}{|c|}{ Argon } & & & \\
\hline 400 & 4.0 & 3.3 & 6.0 & 4.8 & 3.9 & 3.2 & 6.2 & 4.7 & $\ldots$ & & \\
\hline 600 & 4.3 & 3.7 & 5.9 & 5.1 & 4.0 & 3.4 & 6.0 & 5.1 & & & \\
\hline 800 & 4.4 & 3.9 & 5.3 & 4.6 & 4.1 & 3.6 & 5.4 & 4.7 & & & \\
\hline 1000 & 4.3 & 3.9 & 4.5 & 3.9 & 4.0 & 3.6 & 4.7 & 4. J & & & \\
\hline \multicolumn{5}{|c|}{ Hydrogen } & \multicolumn{4}{|c|}{ Krypton } & & & \\
\hline 400 & 1.35 & 1.13 & 1.25 & 0.98 & 4.7 & 3.8 & 7.8 & 5.7 & & 7.1 & 5.5 \\
\hline 600 & 1.43 & 1.25 & 1.58 & 1.37 & 5.0 & 4.3 & 7.5 & 6.2 & & 6.9 & 5.8 \\
\hline 800 & 1.56 & 1.40 & 1.91 & 1.69 & 5.1 & 4.5 & 6.6 & 5.5 & & 6.1 & 5.2 \\
\hline 1000 & 1.73 & 1.57 & 1.94 & 1.71 & 5.0 & 4.4 & 5.5 & 4.5 & & 5.1 & 4.3 \\
\hline \multicolumn{5}{|c|}{ Deuterium } & \multicolumn{4}{|c|}{ Xenon } & & & \\
\hline 400 & 1.86 & 1.57 & 1.85 & 1.50 & 6.1 & 4.9 & 9.8 & 7.4 & & 8.3 & 6.2 \\
\hline 600 & 1.89 & 1.66 & 1.83 & 1.63 & 6.6 & 5.5 & 9.4 & 7.5 & & 8.3 & 6.8 \\
\hline 800 & 1.89 & 1.70 & 1.83 & 1.65 & 6.7 & 5.8 & 8.1 & 6.3 & & 7.4 & 6.1 \\
\hline 1000 & 1.89 & 1.72 & 1.87 & 1.64 & 6.5 & 5.7 & 6.8 & 5.5 & & 6.1 & 5.1 \\
\hline
\end{tabular}

a Maximum value of the impact parameter.

$\mathrm{b}$ Relative efficiencies when $\leqslant$ was estimated from the Goldschmidt values "reference 3:).

$\because$ Relative efficiencies when $\subseteq$ was estimated from Lennard-Jones $\sigma$ values (reierence 32 ).

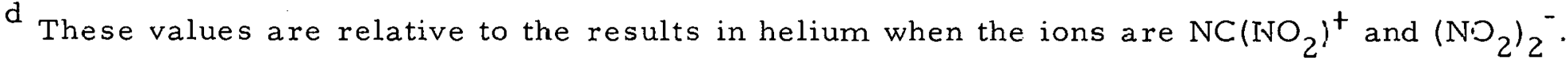


Table XVIII. Relative third-body efficiencies when $E_{D}=0.5 k_{B} T$

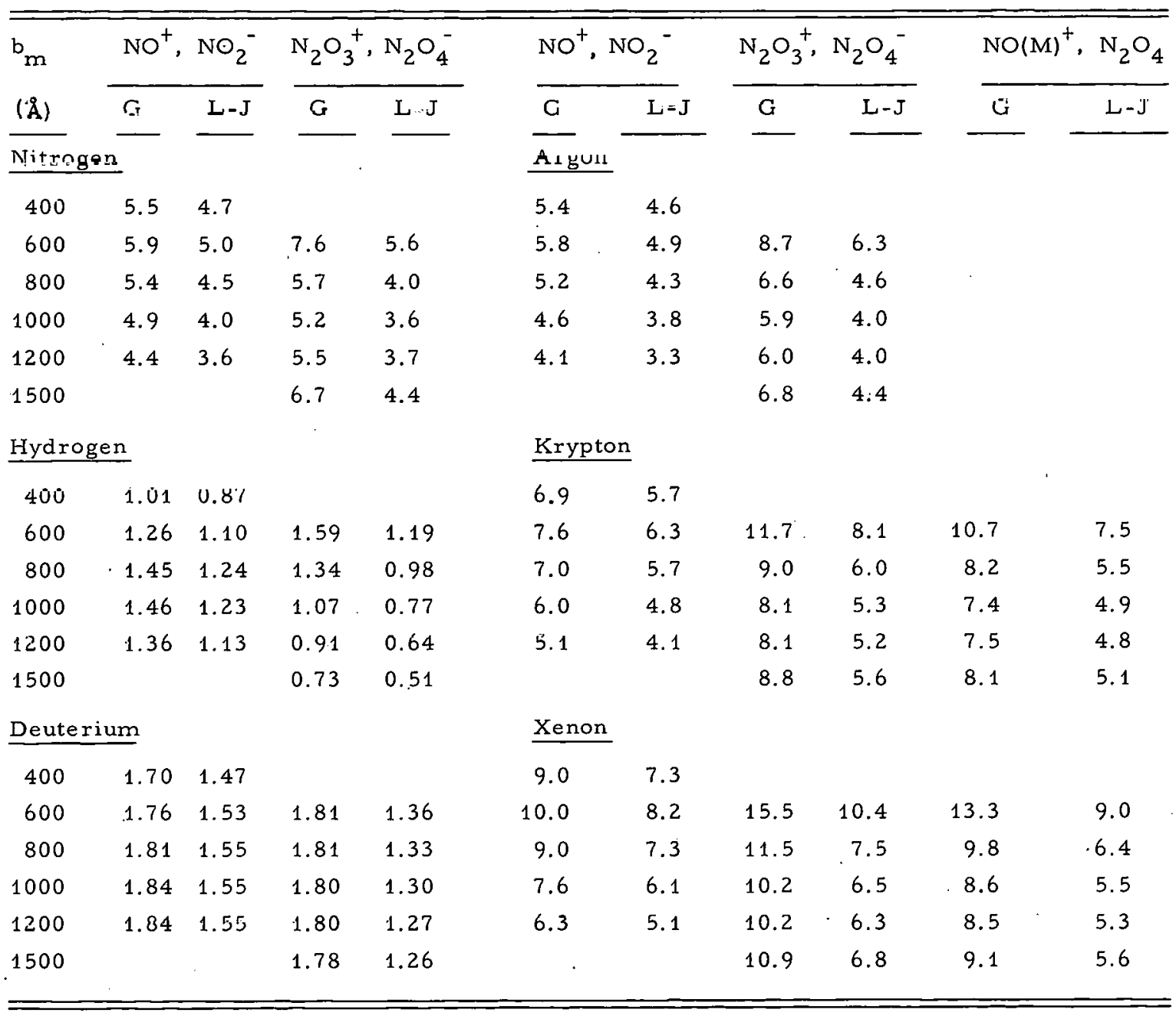


Table XIX. Relative third-body efficiencies when $E_{D}=k_{B} T$

\begin{tabular}{|c|c|c|c|c|c|c|c|c|c|c|}
\hline \multirow{2}{*}{$\begin{array}{l}u_{m} \\
(\AA)\end{array}$} & \multicolumn{2}{|c|}{$\mathrm{PNO}^{\prime}, \mathrm{PJO}_{2}$} & \multicolumn{2}{|c|}{$\mathrm{I}_{2} \mathrm{O}_{3}^{+}, \mathrm{Is}_{2} \mathrm{O}_{4}^{-}$} & \multicolumn{2}{|c|}{$\mathrm{NOO}^{+}, \mathrm{NO}_{2}^{-}$} & \multicolumn{2}{|c|}{$M_{2} \omega_{3}^{+}, M_{2} 1_{1}^{\prime}=$} & \multicolumn{2}{|c|}{$\mathrm{INH}_{-}^{-}(\mathrm{N})^{+}, \mathrm{IN}_{\mathrm{C}^{-1}}$} \\
\hline & G & L-J & $\mathrm{G}$ & L-J & G & $L-J$ & $\mathrm{G}$ & $\mathrm{L}-\mathrm{J}$ & $G$ & L-J \\
\hline \multicolumn{11}{|c|}{ Nitrogen } \\
\hline 600 & 6.3 & 5.2 & 7.4 & 5.0 & 6.1 & 5.0 & 9.0 & 6.1 & & \\
\hline 800 & 5.3 & 4.4 & 7.4 & 4.9 & 5.1 & 4.2 & 8.4 & 5.6 & & \\
\hline 1000 & 5.0 & 4.1 & 9.2 & 6.1 & 4.7 & 3.9 & 9.9 & 6.5 & & \\
\hline 1200 & 5.2 & 4.3 & 11.8 & 8.0 & 5.0 & 4.0 & 12.4 & 8.2 & & \\
\hline 1500 & 5.4 & 4.4 & 13.4 & 8.8 & $5: 1$ & 4.1 & 14.2 & 9.3 & & \\
\hline \multicolumn{5}{|c|}{ Hydrogen } & \multicolumn{2}{|c|}{ Krypton } & & & & \\
\hline 600 & 1.23 & 1.03 & 1.09 & 0.77 & 8.2 & 6.5 & 13.0 & 8.3 & 11.8 & 7.5 \\
\hline 800 & 1.15 & 0.96 & 0.82 & 0.58 & 6.5 & 5.2 & 12.0 & 7.5 & 10.8 & 6.8 \\
\hline 1000 & 0.96 & 0.80 & 0.70 & 0.49 & 5.8 & 4.7 & $1,3.6$ & 8.5 & 12.3 & 7.7 \\
\hline 1200 & 0.00 & 0.73 & 0.70 & 0.47 & 5.7 & 1.7 & $16: 5$ & 10.3 & 11.7 & 9.3 \\
\hline 1500 & 0.86 & 0.71 & 0,70 & 0.49 & 6.0 & 4.8 & 18.5 & 11.5 & 16.3 & 10.3 \\
\hline \multicolumn{5}{|c|}{ Deuterium } & \multicolumn{2}{|c|}{ Xenon } & & & $=$ & \\
\hline 600 & 1.73 & 1.16 & 1.76 & 1.25 & 10.7 & 8.4 & 17.8 & 10.9 & $14: 5$ & 9.0 \\
\hline 800 & 1.74 & 1.47 & 1.73 & 1.23 & 8.3 & 6.6 & 15.8 & 9.6 & 13.3 & 8.1 \\
\hline 1000 & 1.73 & 1.46 & 1.72 & 1.22 & 7.3 & 5.8 & 17.6 & 10.6 & 14.8 & 9.0 \\
\hline 1200 & 1.74 & 1.46 & 1.72 & 1.22 & 7.3 & 5.8 & 20.8 & 12.6 & 17.0 & 10.3 \\
\hline 1500 & 1.76 & 1.47 & 1.72 & 1.22 & 7.3 & 5.8 & 22.9 & 13.8 & 17.4 & 10.6 \\
\hline
\end{tabular}


low pressures, because the fact that most of the ion pairs suffer more than one collision when traveling through the distances considered means that some of our simple assumptions are no longer appropriate. The predictions are illustrated by plotting the values of a predicted for $(a) b_{m}=600 \AA, E_{P}=0$, and $\mathrm{NO}^{+}, \mathrm{NO}_{2}^{-}$ion pairs; (b) $b_{m}=600 \AA$, $E_{D}=0$, and $\mathrm{NO}\left(\mathrm{NO}_{2}\right)^{\ddagger},\left(\mathrm{NO}_{2}\right)_{2}^{-}$ion pairs; and (c) $b_{m}=1500 \AA$ (or larger), $E_{D}=k_{B} \mathrm{~T}$, and $\mathrm{NO}^{+}, \mathrm{NO}_{2}^{-}$ion pairs. The curves are plotted along with the experimental points on Figs. 13 through 16 given in Sec. V. C. The general result is that the experimental points show a greater curvature in the a vs $P$ plots, indicating that the high-pressure predictions of a probably are in errnr.

In conclusion we see that by having the computer do a lot of $\ldots$. work, we can predict the relative third-body efficiencies within limits. In helium, the predictions depend.upon the radius of the hard-sphere core, but the other gases are rather insensitive to this choice. The predicted relative efficiencies do depend upon what requirement we use for deactivation. Also, the predicted efficiencies depend somewhat on the choice of the maximum impacl parameter, $b_{m}$. However, the absolute values of a are strongly dependent on the value of $\mathrm{b}_{\mathrm{m}}$. The procedure used probably gives poor results for pressures as high as a few hundred torr, and it will surely be in error when the pressure is so large that a decreases with increasing pressure.

Thus, the detailed calculation allows one to predict the relative third-body efficiencies; but not the absolute values of a. Also, to get a real test of the detailed calculation, or of any of the other theories, we must have better experimental evidence as to what ions are actually prescnt. 


\section{ACKNOW LEDGMENTS}

This research was directed by Professor Bruce H. Mahan, and it is a pleasure to acknowledge the helpfulness of his interest and suggestions. I have also benefitted from several discussions with Mr. Terry S. Carlton. Finally, I very much appreciate the encouragement of my wife, Dr. Lucy Wu Person, and her considcrable assistance.

Some of the ion collection curves were calculated by using computer time made available by the Computer Center at the University of California.

I also wish to thank the National Science Foundation for three years of support during my graduate study.

This work, wras rnne under the auspices of the U.S. Atomic Energy Commission. 


\section{SYMBOLS}

Primes are used to designate values after the ion-neutral collision.

The subscript $M$ refers to the neutral, the subscript 1 refers to the ion which collides with the neutral, and the subscript 2 refers to the other ion of the ion pair.

\section{Angles}

$\gamma$ is the angle between $V_{m}$ and $V_{2}$,

$v$ is the angle between $V_{m}$ and $V_{m} M^{\prime}$

$t$ is lie diste belween $g$ and $V$.

$\eta$ is the angle between $g_{m} 1 \mathrm{M}$ and $\mathrm{V}_{\mathrm{C}} 1 \mathrm{M}$,

$\theta$ is the angle between $\mathrm{V}_{m}$ and $\mathrm{V}_{m} \mathrm{C}_{1} \mathrm{M}^{\prime}$

$X$ is the angle between $g$ and 'g

$\chi_{12}$ is the angle between $g_{1 M}$ and $g_{m}^{\prime} 1 M$.

$\beta$ is the angle between $V_{m}$ and. $V_{1}^{\prime}$,

and $\phi$ is the angle between the plane of $V_{2}, V_{m}$ and the plane of $V_{m}, V_{1}^{*}$.

\section{Velocities}

$V_{m}, V_{2}, V_{m}$ are the velocities of ion one, ion two, and the neutral.

$g$ is the velocity of the relative motion of the..ion pair.

$g_{1 M}$ is the velocity of the relative motion of the ion-neutral system.

$\mathrm{V}_{\mathrm{C}}$ is the velocity of the center of mass of the ion pair.

$\mathrm{V}_{\mathrm{MC}} 1 \mathrm{M}$ is the velocity of the center of mass of the ion-neutral system. 
APPENDIXES

A. FORTRAN-II PROGRAM TO CALCULATE INDUCED

CURRENT-TIME CURVES

Explanation of Symbols

NR: number of calculations to be done.

IX, IC, Z(I): used to set up cathode ray output.

N'I': number of $\Delta \tau$ steps to be taken.

INF1, oñ more than the number of $\Delta \xi$ в төр.

$\mathrm{MD}$ : the induced current is computed every $\mathrm{MD} \cdot \Delta \tau$. steps.

MN: intermediate values of the ion concentration are printed out every $M N \cdot M D \cdot \Delta_{T}$. stéps.

GA: $\quad G A=G$.

$\mathrm{DT}: \mathrm{DT}=\Delta \tau$.

AL: $A L=\Omega$.

$C N(I): C N(I)=P_{-}(i \Delta \xi)=P_{+}(1-i \Delta \xi)$.

$\mathrm{TU}: \mathrm{TU}=-\Delta \tau / \tau_{\mathrm{RC}}$.

CT: average value of $P_{-}$at some specified $\tau$.

TIC: reduced concentration of ions collected at the anode.

TJD: reduced concentration of ions measured by the induced current.

$A V: A V=W$.

$\mathrm{X}(\mathrm{I}): \mathrm{X}(\mathrm{I})=\mathrm{U}(\mathrm{i} \Delta \xi)=\mathrm{U}(1-\mathrm{i} \Delta \xi)$.

CJN(L): reduced ion current collected at the anode at $L \cdot \Delta \tau$.

POSTID, CAMERA, PICTR: subroutines used for cathode ray output.

$\operatorname{DJI}(\mathrm{L}): \operatorname{DJI}(\mathrm{L})=\mathrm{I}(\mathrm{L} \cdot \Delta \tau)$

* See also Eqs. (D-10) and (D-11) in Sec. IV. A. 
ION COLLECTION RATES (SYM) (INDUCED)

DIMENSION CN $(102), X(102), 0 J 1(502), C J N(502), 0 \times(102), \times 0(102)$,

$12(16), A B S(502), C I L(102)$, CNS $(52)$

COMMON ABS,DJI,PS,DT,LMP, L,CN,DPI,NPI, IXS

DJI $(1)=0$.

$C J N(1)=0$.

$I G=-2$

READ INPUTTAPE 2, 11,NR

11

FORMAT (IS)

DO. $50 \quad K R=1$, NR

READ INPUT TAPE $2.220 .1 \times .16 .12111,1=1.16)$

220 FORMAT (2I5/(9FB.3))

$c$
$c$
$c$

IF IC=NEGATIVE GET GRAPH OF CN VS DISTANCE EVERY IX STEPS,

IF IC=0 GET NO CRT OUTPUT, IF IC=POSITIVE GET GRAPH OF INDUCED CURRENT VS TIME

READINPUTTAPE 2, $12, N T, N P 1, M P, M D, M N, G A, D T, A L,(C N(1), 1=1, N P 1), T U$

12 FORMATII15,4I5.1P3E12.4/(6E12.4))

$C$ MP MUST EQUAL $(N P 1+1) / 2$

$D \times(M P)=0.0$

$X Q(M P)=U$.

$N P=N P 1-1$

$N P M=N P-1$

$N P M 2=N P-2$

$B N P=N P / 2$

$B N 3=B N P * 3.0$

$M P S=M D$

$P S=M P S$

$M N S=M N$

$L M=0$

$T J N=0 \cdot \dot{U}$

$T I J=0.0$

$M P 1=M P+1$

$M P 2=M P+2$

$M P 4=M P+4$

$M P D=M P+M P$

$D N P=N P$

$D P I=1.0 / O N P$

$D N 3=D P I / 3.0$

$D T M=D T * P S / 2.0$

$G A V 3=G A * D N 3$

$D T A L=D T * A L$

$D T Y=D T / D P I$

I $X S=I X$

WRITE OUTPUT TAPE 3,13,NP,NT, GA,DT, AL,TU,ONP,ON3,DTM,ICNII),

$1|=1, N P| 1$

13 FORMAT 13 I3H INPUT DATA 215,1 P6E $15.4 / 18 E 14.4) 1$ DO $404 \quad I=M P 1, N P 1$

$I M=M P D-I$

$404 \quad D X(I)=C N(I M)-C N(I)$

C CALCULATE NEW VAlues of $x$

$410 \quad \forall J=J$

$A V=1.0-2.7182818 *(V J * T U)$

$X Q(M P 1)=D X(M P 1) * G A V 3$

$D O 420 \quad I=M P 2$. NP 1

$420 \times 0(1)=X 0(1-2)+(D \times(1-2)+4.0 * D X(1-1)+D X(1)) * G A V 3$

$X T 4=X O(M P 1)+X O(N P)$ 
$X T 2=\times 0(M P 2)$

DO $426 \quad I=M P 4, N P, 2$.

$X T 4=X T 4+X Q(I-1)$

$426 \times 12=x T 2+x 011$

$X O T=2 . U * X T 2+4 . U * X T 4+X O(N P 1)$

$X(M P)=A V+X Q T / B N 3$

IF $(X(M P)) 428,430,430$

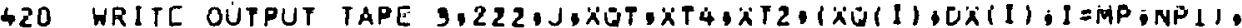

1 (CN! I), I I, ISP I) X XIMP)

222 FORMAT (9HOX(MP)NEG, $115 /(1 H 0,1 P 8 E 14.5))$

$$
\text { GO TO } 50
$$

$4300032 \quad[K=M P 1, N P 1$

$X(I K)=X(M P)-X Q(I K)$

$I F(X \mid I K)) 31,32,32$

31 WRITE OUTPUT TAPE $\$, 2 \angle 4, I K, J, X(S),(X U 111,1) \times(1), 1 \equiv M H, N H 1)$,

$1 \quad(C N(1) \cdot I \equiv 1 \cdot N P 1) \cdot\left(X(1) \cdot I=M P, \mid K_{1}\right)$

224 FORMAT(GHUNEGXI,14,2H),115/(1HO,2P8E14.5))

GO TO 50

32 CONTINUE

IF $(J-1) \quad 381,381,33$

33 IF (MD-J) $34,34,40$

$34 M D=M D+M P S$

$L M=L M+1$

$L M P=L M+1$

C. CALCULATE INOUCED CURRENT

$C J N(L M P)=X(N P 1) * C N(N P 1)$

$T J N=T J N+C J N(L M)+C J N(L M P)$

$D J Z=X(M P 2) *(C N(M P 2)+C N(M P-2))+X(M P)=C_{N}(M P)$

$D J 4=x(M P 1) *(C N(M P 1)+C N(M P-1))+X(N P) *(C N(N P)+C N(2))$

DO $35 \quad I=M P 4, N P, 2$ $I M=M P D-1$

$0 J 4=034+x(I-1) *(C N(I-1)+C N(I M+1))$

3). UJL=UJL+X(1) $(C N(1)+C N(I M))$

$D J I(L M P)=12.0 * 0 J 2+4.0 * D J 4+X(N P 1) *(C N(N P I)+C N(1))) / B N 3$

II $J=\mid\lfloor J+U J|(L M)+U J|(L M H)$

I $F(M N-L M) 38,38,40$

$38 \quad M N=11 N+M N S$

$3 R ! \quad C N T 4=C N(Z)$

$C N T^{2}=U .0$

QQ $391=4, N P, 2$

CNT2 $=$ CNT2+CNI1-1

39 CNT4 $=$ CNT4+CN(I)

$C T=(C N(1)+4.0 * C N T 4+2.0 * C N T 2+(N(N P 1)) * D N 3$

$T I C=D T M * T J N$

$T J D=D T M * T I J$

WRITE OUTPUT TAPE 3,202 , J,NPI,MNS,MPS,CT,TIC,TJD,AV, ICNII, I 1 ,

1 NP1). ( $(x(1), 1=M P, N P 1)$

202 FORMAT 1 (HO,215,14HCP,CN,X,AT DTX 2I5,5X,1IHTOTAL IONS=

1 1PIE19.4,5X,13HIONS COLLECT $=1 E 19.41$ 1HO,16HINDUCEO ION COL =

2 IE16.4.5X,4HVOLT 1E16.4/11HO,6E19.41)

IF (IX-J) $391,391,406$

391 IFIIC) $393,406,406$

393 CALL CAMERA (IG, IX)

406 IF (NT-J) $44,44,40$

$c$

CALCULATE NEW V.ALUES OF CN

$40 \mathrm{C}$ CILII) $=$ CN(I)*X(NPI)*DTY 


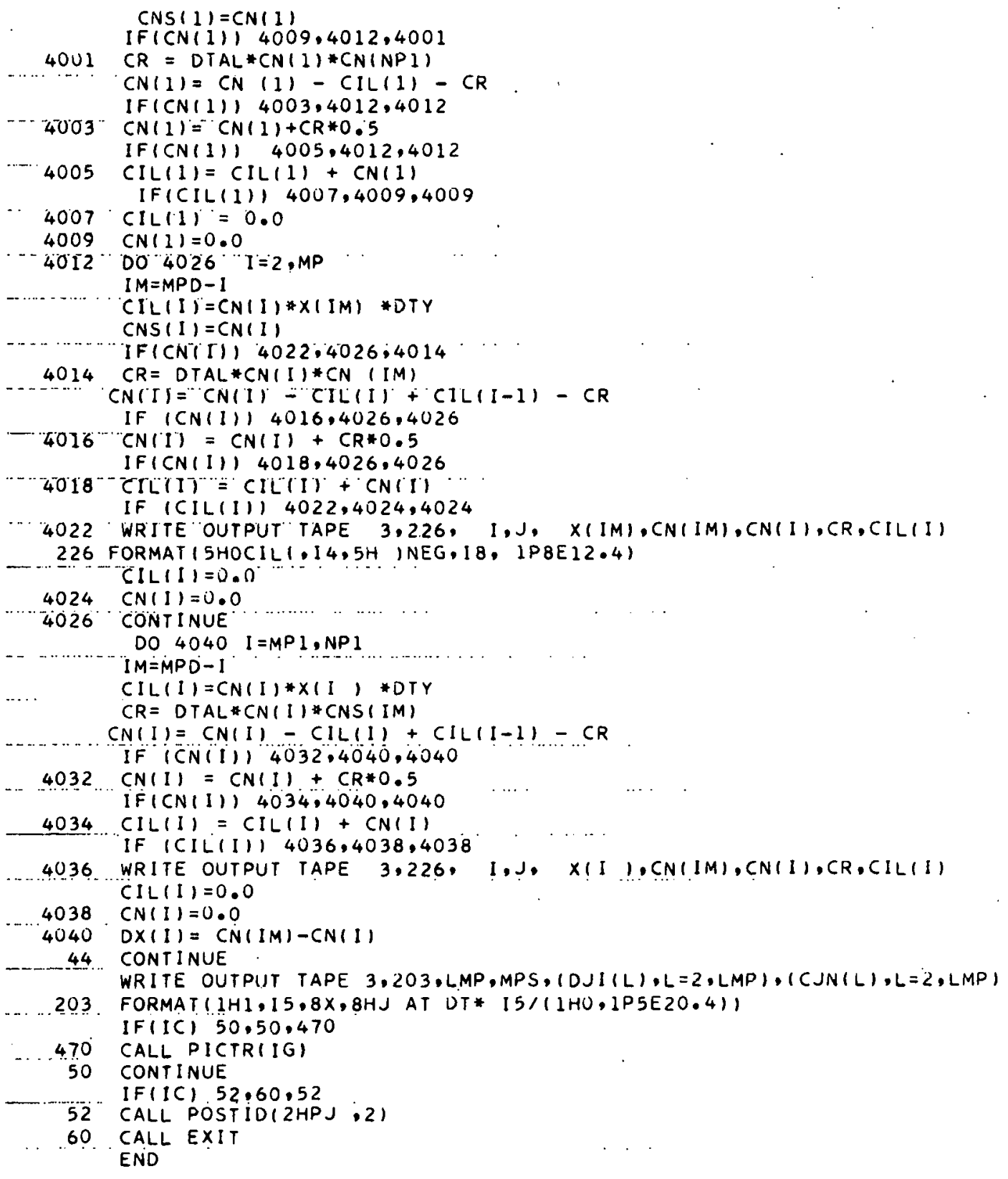


B. RATE OF OBTAINING DEFLECTION ANGLES IN $\Delta x_{1 M}$ REGIONS

Listed below are the values of $\Delta Y$ for various $\Delta\left|\left(X_{1 M}-m 2 \pi\right)\right|$ regions at different values of $Z$. The rate constant, $k_{X}$, for formation of the region $\Delta\left|\left(x_{1} M^{-m 2 \pi}\right)\right|$ is given by

$$
k_{X}=\pi e\left(a_{M} / \mu_{1 M}\right)^{1 / 2} \Delta Y \text {, }
$$

where $e$ is the electronic charge, $a_{M}$ is the polarizability of the neutral, and $\mu_{1 \mathrm{M}}$ is the ion-neutral reduced mass.

\begin{tabular}{|c|c|c|c|c|c|c|c|c|}
\hline \multirow{2}{*}{$\begin{array}{c}\Delta /\left(x_{\left.1 M^{-m 2 \pi}\right)}\right. \\
(\mathrm{deg}) \\
\end{array}$} & \multicolumn{8}{|c|}{$\mathrm{Z}=\left(\mu_{1 \mathrm{M}} / \mathrm{a}_{\mathrm{M}}\right)^{1 / 2} \mathrm{~g}_{1 \mathrm{M}^{\mathrm{s}}}{ }^{2} / \mathrm{e}$} \\
\hline & 0.30 & 0.90 & 1.55 & 2.0 & 3.0 & 4.0 & 6.0 & 8.0 \\
\hline 21010. & 4.51 & 4.51 & 4.51 & 4.49 & 4.49 & 4.18 & 2.25 & 0.302 \\
\hline 10 to 20 & 1.104 & 1.135 & 1.099 & 1.087 & 0.741 & 0.118 & 0.137 & 0.202 \\
\hline 20 to 30 & 0.532 & 0.563 & 0.523 & 0.527 & 0.145 & 0.174 & 0.233 & 0.257 \\
\hline 30 to 40 & 0.352 & 0.372 & 0.346 & 0.272 & 0.179 & 0.222 & 0.312 & 0.404 \\
\hline 40 to 50 & 0.269 & 0.278 & 0.271 & 0.1 .59 & 0.210 & 0.266 & 0.382 & 0.502 \\
\hline 50 to 60 & 0.222 & 0.225 & 0.237 & 0.169 & 0.235 & 0.302 & 0.439 & 0.577 \\
\hline 60 to 80 & 0.361 & 0.363 & 0.332 & 0.371 & 0.523 & 0.678 & 0.996 & 1.317 \\
\hline 80 to 100 & 0.290 & 0.293 & 0.310 & 0.381 & 0.544 & 0.713 & 1.054 & 1.398 \\
\hline $10 n+012 n$ & 07.38 & 0.2 .38 & 0.282 & 0.350 & 0.506 & 0.665 & 0.988 & 1,312 \\
\hline 120 to 140 & 0,191 & 0.181 & 0.225 & 0.281 & 0.410 & 0.540 & 0.804 & 1.068 \\
\hline 140 to 160 & 0.142 & 0.116 & 0.144 & 0.181 & 0.266 & 0.352 & 0.524 & 0.697 \\
\hline 160 to 180 & U.11y & 0.057 & 0.052 & 0.065 & 0.093 & 0.122 & 0.182 & 0.242 \\
\hline
\end{tabular}




\section{FORTRAN-II PROGRAM FOR THE DETAILED CALCULATION of $\mathrm{a}_{\mathrm{T}}$}

The program is divided into two parts. The program labeled RATE OF ION-ION RECOMBINATION, together with its subroutine WGTEP, calculates the values of $k_{3}(i)$ for deactivation by collision with the positive or with the negative ion. These values of $k_{3}(i)$ are input data for the program labeled CALC ALPHA, which calculates the values of $a_{T}$, making use of the subroutine AVEK2.

Explanation of Symbols

INUM: number of calculations to be done.

IPM: number of $\Delta X_{1 M}$ regions.

$X B(M, I S)$ : values of $X_{1 M}$.

IS, L ZT, ZILM(L3), BAM(MA, IS): data which give the rate constant for obtaining the $x_{1 M}$ deflections.

NP3: number of steps used in computing the $\epsilon(\rho)$ distribution.

TF1, TF2, TM: $T_{1}(\infty), T_{2}(\infty)$, and $T_{M}\left(r_{1 M}=\infty\right)$, respectively, in units of $k_{B} T$.

TEMP: temperature in ${ }^{\circ} \mathrm{K}$.

AM1(MIJ), AM2(MIJ): gram molecular weights of ions one and two.

NN: number of $\Delta \gamma(\infty)$ and $\Delta \epsilon(\rho)$ steps.

$N F$ : index used to specify $\gamma(\infty)$ value.

$\mathrm{KM}:$ number of $\Delta v$ steps.

$C E(N)$ : values of $\gamma(\infty)$ and $\epsilon(\rho)$.

$\mathrm{CK}(\mathrm{K})$ : values of $v$.

$E D: E_{D}$ in units of $k_{B} T$.

MM: number of different neutrals.

$\operatorname{AMM}(M)$ : gram molecular weights of the neutrals.

$N X$ : number of $\Delta b$ steps.

$B(J B)$ : values of $b$.

L, $T$ : number of different values of $s$.

L9: index used to specify value of $s$. 
$Q L(M, L, M I):$ values of $\lambda$ as defined by Eq. (D-29) in Sec. IV.C.

ARM: gram molecular weight of $\mu_{1 M}$.

TIN, TR, TC: TIN $=T_{1}(\rho), T R=T_{R 1 M}, T C=T_{C 1 M}$.

$X, C S E, S N E: \quad X=X, C S E=\cos \eta, S N E=\sin \eta$.

TRR, CSG: $T R R=T_{R}(\rho), C S G=\cos \gamma$.

EEP: $e^{2} / \rho$ in units of $k_{B} T$.

$R O=R(J, N F): \quad R O=r_{C}\left[b_{j}, \gamma(\infty)\right]$.

TCI, TRF: TCI $=T_{C}, \quad T R F=T_{R}(\infty)$.

FPT: fraction of collisions with $\pm x_{1 M}$ that give deactivation.

$X T(T R), R \Pi(T B), D E(I B): X I=X_{1 M I}, B D=\beta, D E=\Delta I^{\prime}{ }_{1}(\rho), \quad L^{\prime} H^{\prime} D A(L 9):$

deactivation rate constant for a particular value of $\nu$.

FFTDA(L9): deactivation rate constant for a particular value of $\epsilon(\rho)$.

ETDA(L9): used as data in CALC ALPHA; k.., fnr deartivation by collision with one of the ions is $k_{3}(i)=\pi e\left(a_{M} / \mu_{1 M}\right)^{1 / 2} \operatorname{ETDA}$.

$\operatorname{GFP}(N F): \operatorname{GFP}=g(\gamma(\infty))$.

$G C(N F, T 3(N F), A A(N F)$ : used as data in CALC ALPHA to calculate $\tau(i, j)$.

WGTEP: subroutine used to calculate the distribution of $\epsilon(\rho)$ and the weighting factor for the $\Delta \epsilon(\rho)$ regions.

EPI: $\quad \operatorname{EPI}=\epsilon(\infty)$.

WL(NN): woighting factnr for the $\Delta \epsilon$ regions.

Additional Symbols in CALC ALPHA

NP, PRS(NM): the total number and the values of the pressures at. which $a_{T}$ is calculated; INIVI is the indes specifying tho pressure.

JS: smallest value of $j$ of the $b_{j}$ used.

JM, JL: first and last values of $m$ for the $b_{m}$.

POL: $a_{M}$ in cgs units.

$\mathrm{ZJE}(\mathrm{J})$ : number of steps taken for each $\mathrm{j}$ in the calculation of $\langle 1 / \tau(i, j)\rangle$.

$\operatorname{FDI}(J, J 3): \quad F D I=f(J 3, J)$.

$\operatorname{DAL}(\mathrm{I})$ : contribution to $a_{\mathrm{T}}\left(b_{\mathrm{m}}, \gamma(\infty)\right)$ from ion pairs in the Ith state. $\operatorname{ALF}(L 9, N M, N F): \quad A L F=a\left(b_{m}, \gamma(\infty)\right)$. 
$A K Z(L 9, N M, N F): A K Z=k_{0}(\gamma(\infty))$.

$\operatorname{ALPH}(L 9, N M): A L P H=a_{T}$.

$\operatorname{AVKZ}(L 9, N M): A V K Z=k_{0}$.

$\operatorname{RELE}(J, L 9)$ : relative third-body efficiency when $b_{m}=b_{J}$. AVEK2: subroutine used to calculate $\langle 1 / \tau(i, j)\rangle$.

$\mathrm{DT}(\mathrm{J} 5)$ : values of $\tau$ for $\mathrm{b}^{2}=\mathrm{J} 5: \Delta \mathrm{b}^{2}$.

$\mathrm{SKI}: \mathrm{SKI}=\pi \mathrm{gb}_{\mathrm{m}}^{2}$ :

$\operatorname{AKJ}(J, N F): \quad \operatorname{AKJ}=\langle 1 / \tau(J, J)\rangle$.

$A K I(J, J 3, N F): A K I=\langle 1 / \tau(J 3, J)\rangle$. 


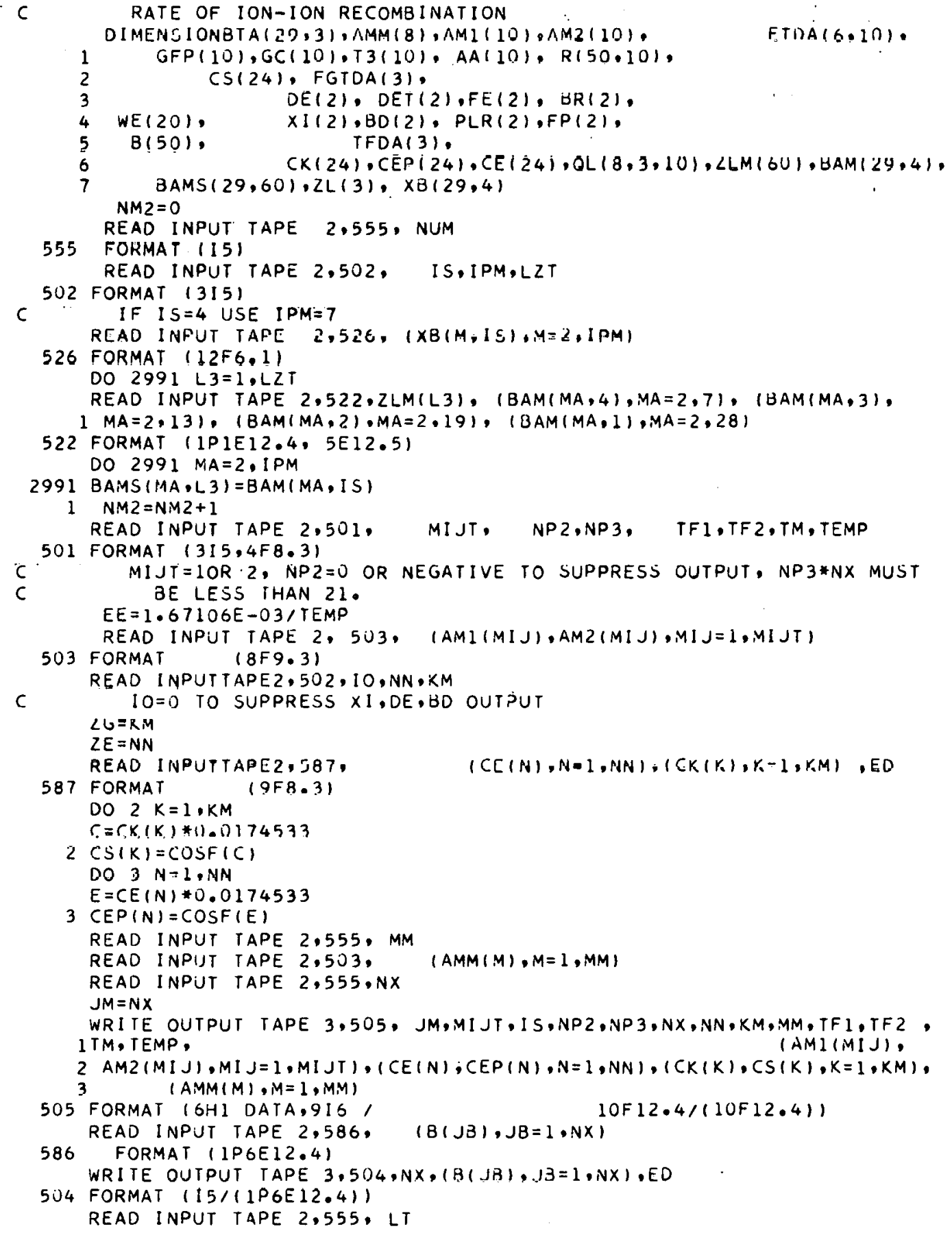




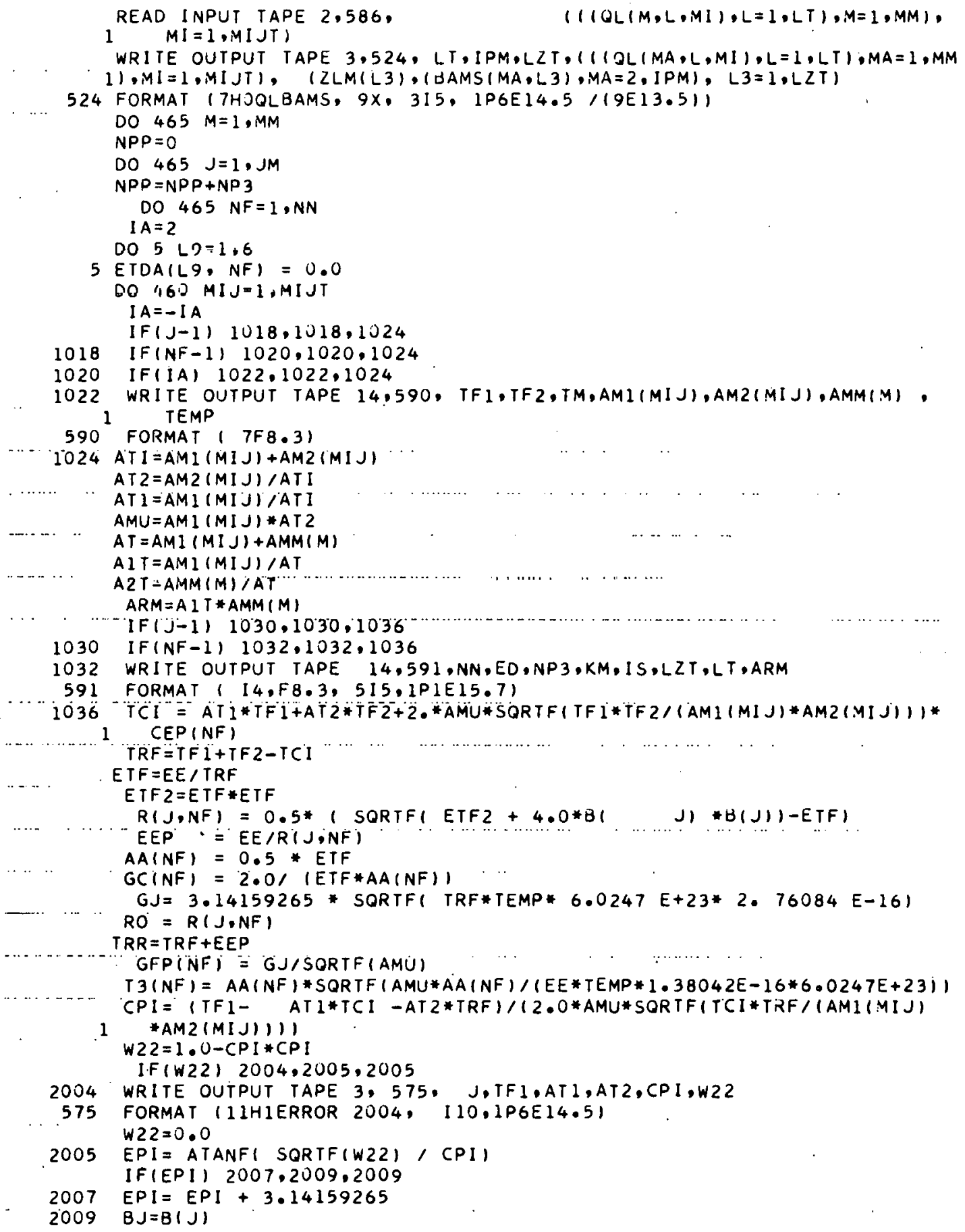




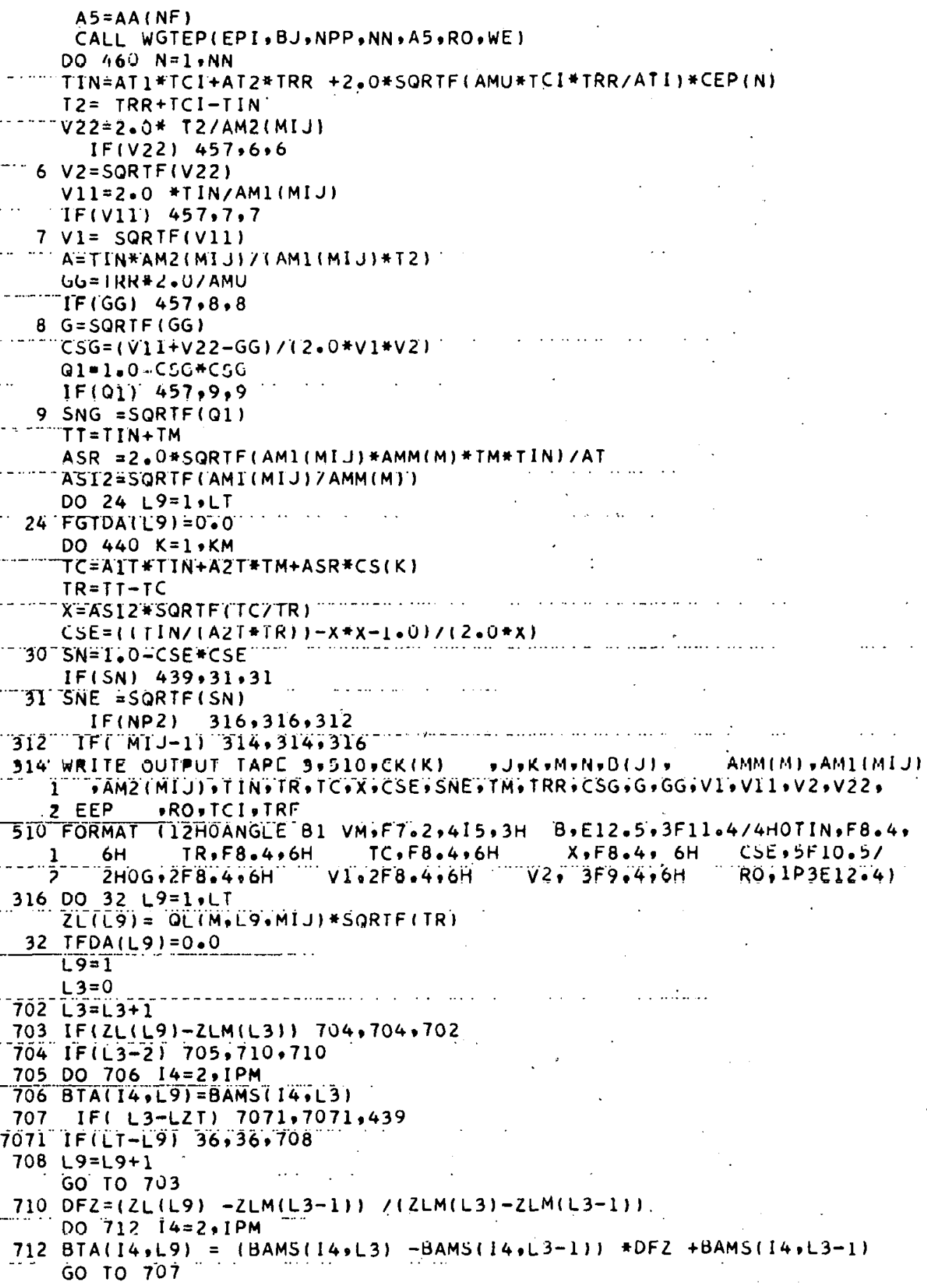


36 IF (NP2) $363,363,361$

361 WRTTE OUTPUT TAPE 3,585, M,J,NF,MIJ,N,K, $(2 L(L 9), L 9=1, L T)$

585 FORMAT $13 \mathrm{HOM}=\mathrm{I}, 14,6 \mathrm{H} \quad \mathrm{J}=14,6 \mathrm{H} \quad \mathrm{NF}=114,6 \mathrm{H} \quad \mathrm{MIJ}, 13,6 \mathrm{H}$ NEP $\because 14,6 \mathrm{H}^{\prime} \cdots \mathrm{K}=, 14,6 \mathrm{H} \quad \mathrm{ZL}=.3 \mathrm{~F} 15.61$

363 I $x=1$

$37^{-1} \mid x=1 x+1$

$X I(1)=X B(1 X, I S)$

$X I R=X I T \quad 11 * 0.0174533$

$I B=1$

$42 D E(\quad I B)=2.0 * X * T R * A 2 T *(C S E *(1.0-\operatorname{COSF}(X I R))+\operatorname{SNE} * \operatorname{SINF}(X I R))$

DETI $\quad(B)=D E(\quad I B I / T I N$

FET IBIEI:D-DET( IB)

IF(FEI IB) $144,43,43$

$43 T N B=(S I N F T X I R) *(1,+X * C S E)+X * S N E *(\operatorname{COSF}(X I R)-1.0)) /(X *(X+C S E)+$ $1 \operatorname{COSF}(X I R) *(1 \cdot 0+X * C S E)-X * S N E * S I N F(X I R))$

$B I=A T A N F(T T N B)$

IF $(X-1.000,45,44,44$

$44^{-} B R(I B)=B I$ GO TO 55

45 IF TIX-2) $44,44,46$

46 IF (BRIIB) $47,44.52$

$47^{-}$IF (BBI)- $4 \overline{9}, 48,550$

$48 B R(1 B)=3.14159265$ GO TO 55

49 IF $(B R(I B)+1.5708 \quad 154.54 .044$

50 BRTI8I $=73.14159265$ 60 TO 55

$-52^{-}$IF $(B 1)^{-} 54 ; 40,53$

53 IF (BR (IB) -1.5708

$54-B R^{-}(I \bar{B} T=3.14159265$

$55 \mathrm{BD}(\mathrm{B})=\mathrm{BR}(\mathrm{B}) / 0.0174533$

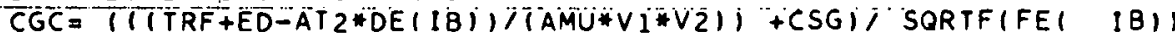
IF (CGC-1.0) $105,160,160$

105 IF $T C G C+1001150,150.120$

$110 B G C=\operatorname{COSF}(B R(I B)) * C S G$ BGSESTNFTBRTIBTT SNG IF (BGS) $112,170,130$

$112 B G S=-B G S$

$130 \mathrm{CGT}=8 \mathrm{BC}+\mathrm{BGS}$ IFICGT-CGCI 160.160 .132

$132 \mathrm{CGT}=\mathrm{BGC}-\mathrm{BGS}$

IF ${ }^{\prime}(C G T-C G C)-134,150 \% 150$

$134 C P=(C G C-B G C) / B G S$ $05=1.0-C P * C P$

IF(O5) $144,136,136$

$\left.1 \overline{3} 6^{-P L R T} I B\right)=A T A N F(S O R T F(O 5) / C P)$

IFIPLRI I8) $138.140,140$

$[38$ PLRI IBI $=3.14159265+P L R(I B)$

$140 F P(18)=P L R(\quad[B) ! 3.14159265$ GO TO $4: 17$

144 WRITE OUTPUT TAPE 3.506 ,IX,IB,DE I I $81, X, T R, A 2 T, C S E, X I R, S N E$, I DETI 1BI,CGC,BGC;BGS,CP,CGT,FE( 1B),OS

506 FORMAT I 9HOERROR144,9X .215,7F13.7/ 1HO,6F13.7,9X,2F13.71 GO TO 417

$150 F P(1 B)=1.0$

$P\left[R T^{\prime}[B]=3.14159265\right.$

GO TO 417

$160 \mathrm{FP}(\mathrm{IB})=0.0$ 
PLRI $I B]=0.0$.

60 TO 417

170 IF(BGC-CGC) $160,160,150$

$417^{\circ}$ IFIIB-2) $418,420,420$

$418 \quad 1 B=2$

$x I R=-x I R$

XII 2$)=-X I(1)$

GO TO 42

$420 \mathrm{FPT}=(F P(1)+F P(2)) / 2.0$

IF(IO) $421,4210.421$

421 WRITE OUTPUT TAPE $3,511,1 X$,

FPT,

I IBTA $(Y X, L 9), L 9=1,3\},(X I(I B), B D(I B), D E(18), D E T(I B), F E(I B), F P(I H$ 2), $\operatorname{RLR}(1 B), 10=1,2)$

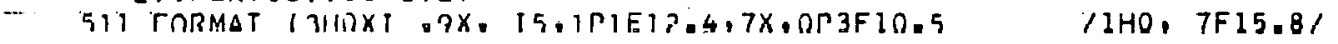

$1 \quad 1 \mathrm{HO}, 7 \mathrm{~F} 15.81$

$4210^{\circ}$ DO 422 L9=1,LT

FDACT $=F P T * B T A(I X, L 9)$

422 TFDA $(L 9)=$ TFDA $(L 9)+F D A C T$

423 IF IIX-IPM) $37,430,430$

430 IF (NP 2) $432,432,431$

431 WRITE OUTPUT TAPE 3.531 , IX,FPT, (TFDA (L9),LQ=1,LT)

53I FORMAT (3HOL9; I5, 4F15.8)

432 DO 434 L9=1,LT

434 FGTDAIL 9$)=$ FGTDA(L9) + TFDAIL 9$) / 20$ GO TO 4398

439 WRITE OUTPUT TAPE 3,514., K, TC,AIT, TIN, A2T, TM, ASR, CSIK), TR,

$1 T T, X, A S 12, C S E, \quad S N,(Z L(L 9), L 9=1,3), J, M, M ! J, N F$

514 FORMAT IIOHIERROR 439, 9X, 15, 7F13.7/1HO, 9F13.7/1H0.4I8)

4398 IFINP2) $440,440,4399$

4399 WRITE OUTPUT TAPE $3,512,(K(K), K, \cdots$ TFGTÖAIL 9 IOLG=1,LYI

512 FORIAAT (3HOCK, 1F8.3,9X,1 15,5X, 2HL9, 3F20.8)

440 CONTINUE IF(IA) $452,452,454$

452 DO 453 L $Q=\dddot{I}, \mathrm{~L} T$

453 ETDA $(L 9, N F)=E T D A(L 9, N F)+F G T D A(L 9) * W E(N)$ (i) in 4 til

454. DO $455 \quad L 9=4,6$

$455^{\circ}$ ETDA (L9,NF) = ETOOAILQ:NF̈I + FGTDAI L9-3) *NE(N) GO TO 460

457 WRITE OUTPUT TAPE 3,515, N.MIJ, NB, TIN, ATI, TCI, AT2,TRR,

$1 \mathrm{AMU}, A T I, C E P(N), A M I(M I J), V 1, V 2, C S G, T R F, R O,(B(J B)$,

2 JB=1,NX);T2, V22, VII, GG, (J!, U2

515 FORMAT (IOHOERROR 457.9X, 315, 7F12.6/ 1HO, 6F12.6, IP3E12.4,

1 (1HO, $9 E 12.41)$

460 WRITE OUTPUT TAPE 3,513, AMI(MIJ),CE(N), AMM(M), (ETDA(L9,NF),L9= $11.6 i$

513 FORMAT $15 H O * M I J, F 8.3 .6 H \quad E P=, F 8.3,6 H \quad M=, F 8.3 .6 H$ ETDA,6F

1 12.81

I $F(N F-N N) \quad 465,4562,4562$

4562 IF(J-1) 4564,4564,4565

4564 WRITE OUTPUT TAPE 14,593, (CE(N4), GFP(N4), GC(N4), T3(N4), AA(N4), 1 N4 $=1, N N$ I

593 FORMAT ( IPIE $11.3,4 E 15.7)$

4565 WRITE OUTPUT TAPE $14,592, B(\mathrm{~J}), 1 R(\mathrm{~J}, \mathrm{~N} 4), \mathrm{N} 4=1, \mathrm{NN})$

592 FORMAT (IP5E 15.7$)$

WRITE OUTPUT TAPE 14,594 , ( (ETDA $(L 9, N 4), L 9=1,6), N 4=1, N N$ )

594 FORMAT (IPIE 12.4.5E 12.5)

465 CONTINUE 


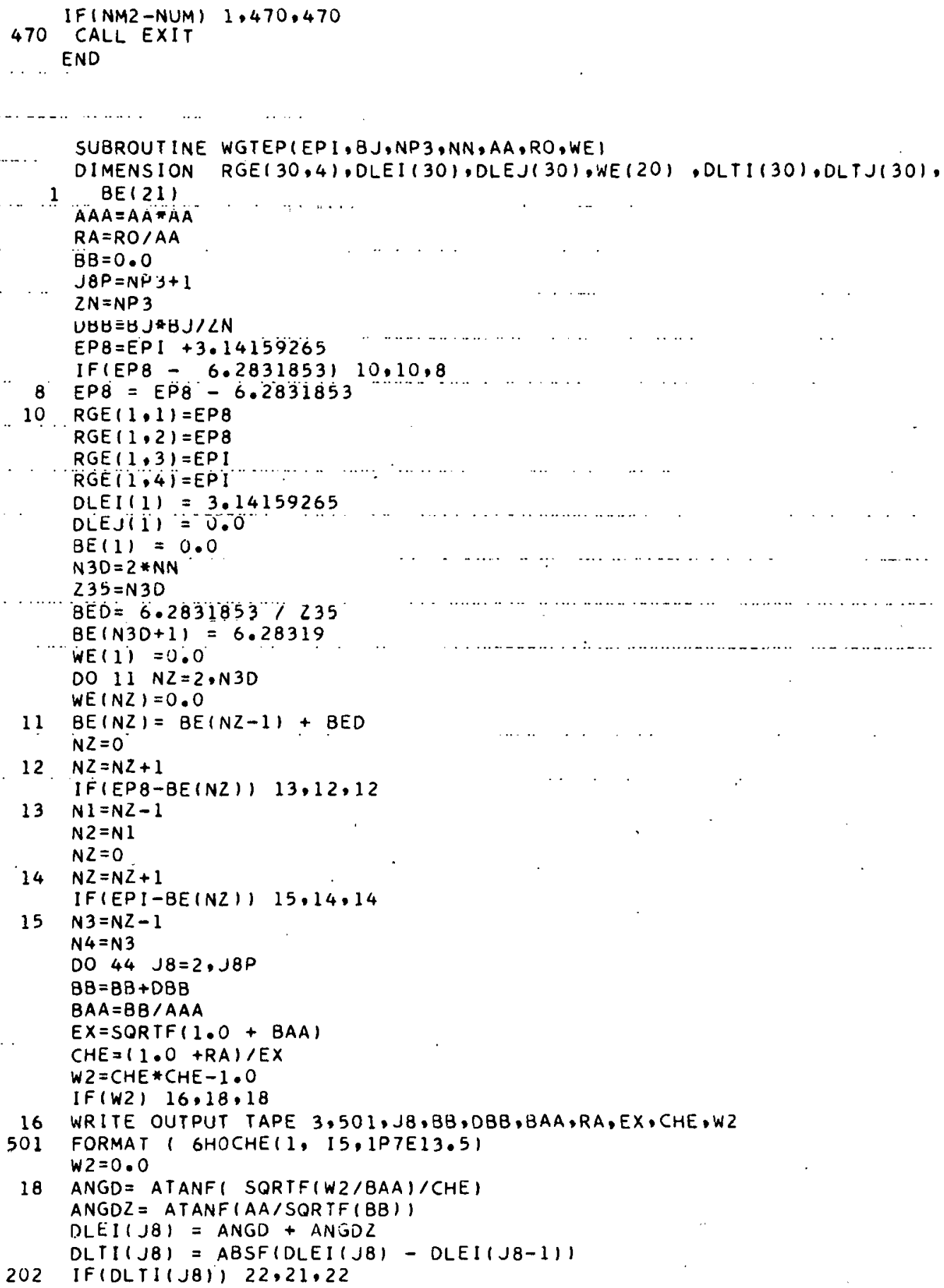




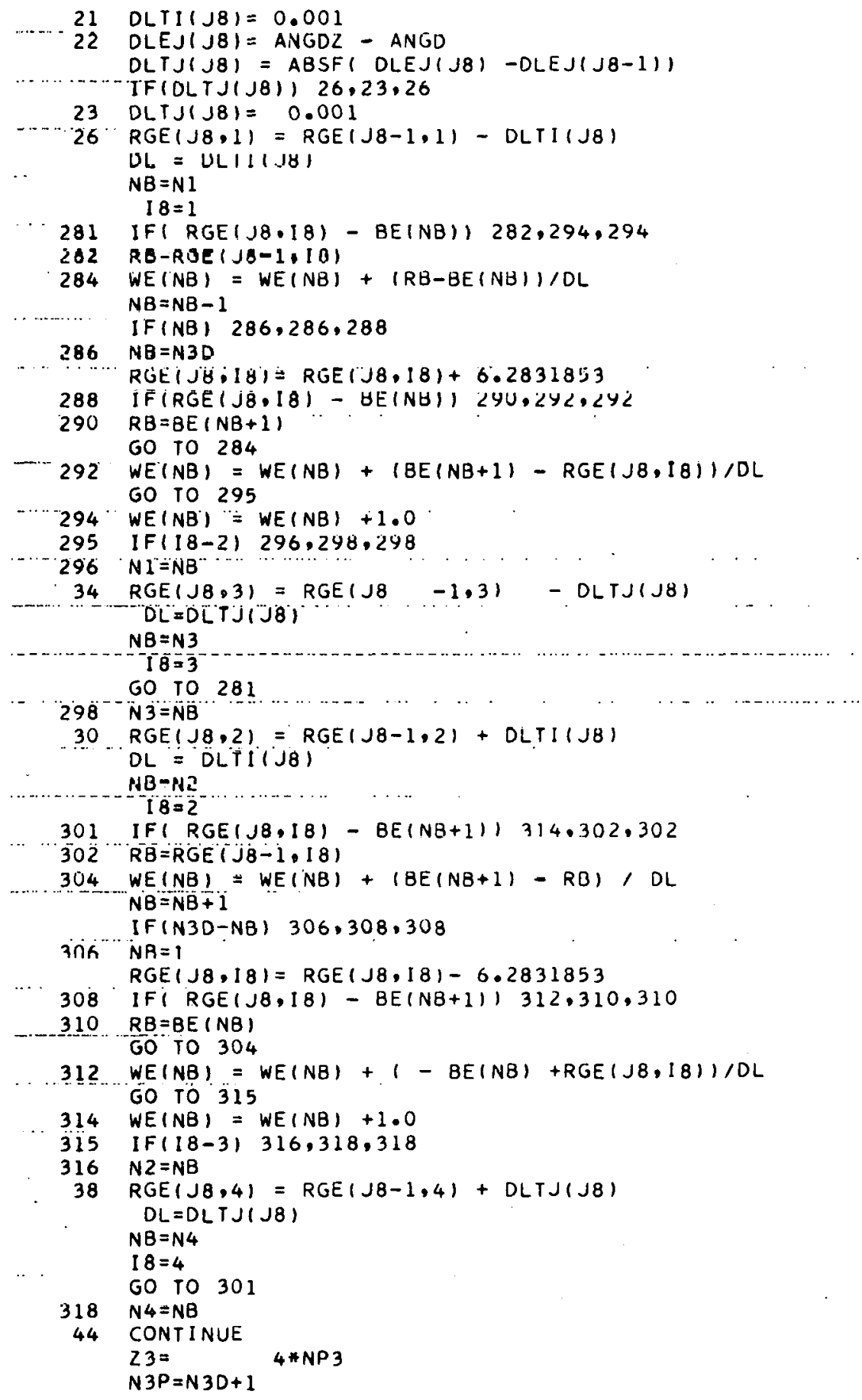




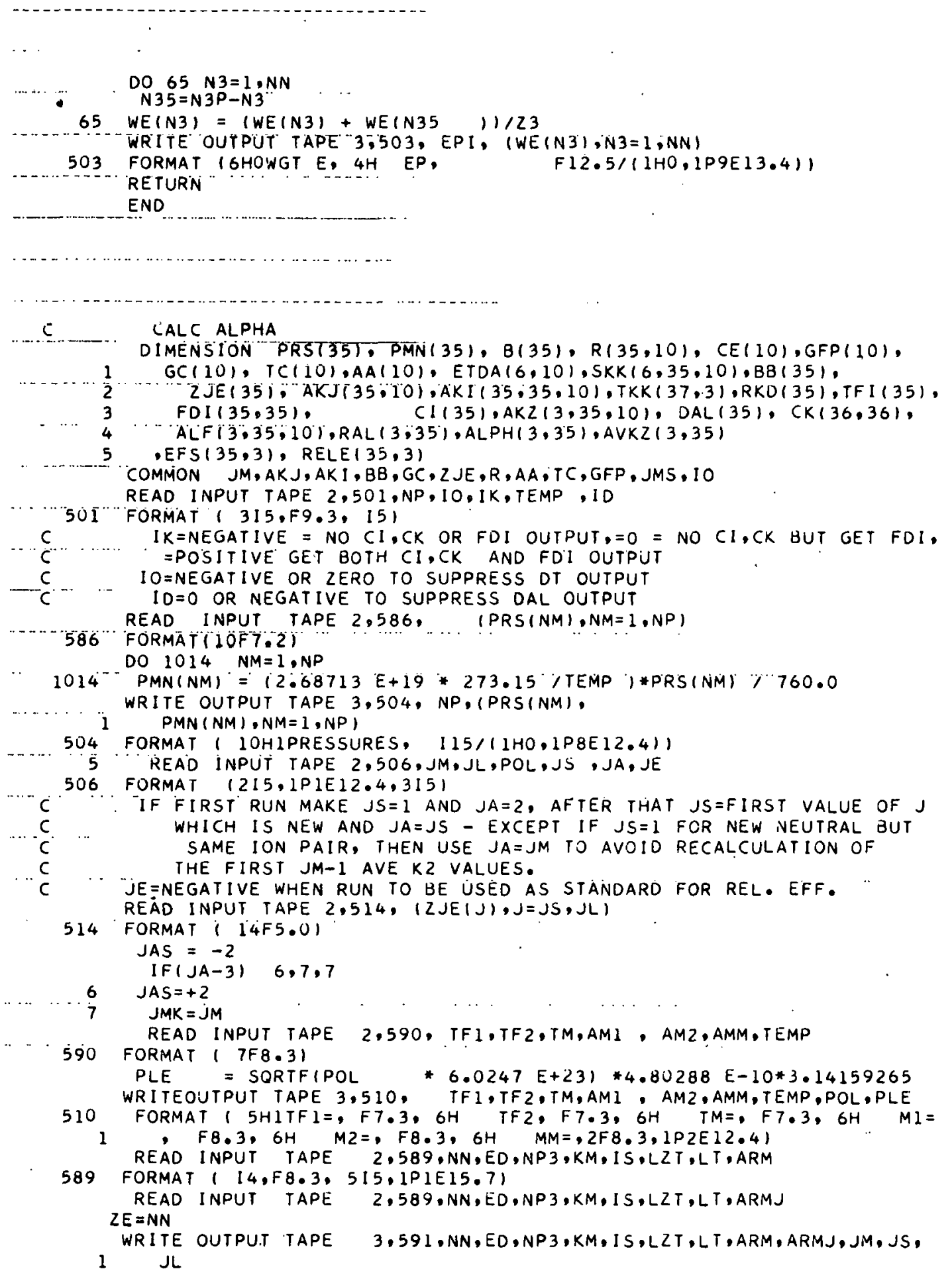


591 FORMAT ( 14,F8.3, 515,1P2E15.7,315)

READ INPUT TAPE 2,593 , (CE (NF), GFP(NF), GC(NF),TC(NF), AA(NF), $N F=1, N N$ I

593 FORMAT ( IPIE11.3.4E 15.7)

WRITE OUTPUT TAPE 3,593, (CE (NF), GFP(NF),GC(NF),TC(NF), AA(NF), $N F=1, N N$

PLRM $=$ PLE / SQRTF (ARM)

PLRMJ = PLE /SORTF (ARMJ)

DO 10 J.JE.JL

READ INPUT TAPE 2. $592, B(),,(R(J, N 4), N 4=1, N N)$

592

REAT (IP5E 15.7)

WRITE OUTMUT TAME 3,592, HIJ), (RI, +N4),NLEI,NN!

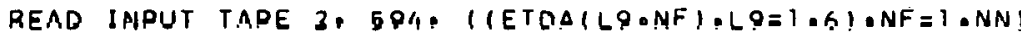

594 FORMAT (IPIE $12.4 .5 E 12.5)$

WRITE OUTPUT TAPE 3,594 , ( (ETDA $(L 9, N F), L 9=1,6), N F=1, N N)$

DO $10 \mathrm{NF}=1, \mathrm{NN}$

DO $8 \quad Q 9=1 \cdot 3$

$5 K K(L 9, J, N F)=E T D A(L 9, N F) * P L R M$

$00 \quad 10 \quad L 9=4.0$

10

$S K K(L 9, J, N F)=E T D A(L 9, N F) * P L R M J$

$J Q=J L-1$

$J M=J M-1$

$D O 90 \mathrm{JB}=J M, J O$

$J M=J M+1$

$J M S=J M-1$

DO $15 \mathrm{~J}=\mathrm{JS}, \mathrm{JM}$

$15 B B(J)=B(J) \quad * B(J)$

DO $60 \mathrm{NF}=1, \mathrm{NN}$

IF(JAS) 37.35 .35

$35^{\circ}$ CALL AVEK2 " (NF; JA)

$37 C I(J M)=G F P(N F) *(B B(J M)-B B(J M S))$

DO $50 L 9=1, L T$

DO $39 J=2, J M$

$39{ }^{\prime}$ TKK $(J, L 9)=\cdots(S K K(L 9, J, N F)+S K K(L 9+3, J, N F)+S K K(L 9, J-1, N F)$

$1+5 K K(L 9+3, J-1, N F)) * 0.5$

DO $50 \mathrm{NM}=1, \mathrm{NP}$

DO $392 \quad J=2, J M$

392 RKD $(J)=T K K(J, L 9) * P M N(N M)$

DO $394 \mathrm{~J}=1, \mathrm{JMS}$

$T F I(J)=0.0$

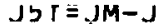

DO $394 \quad J 5=1, J 5 T$

$J \dot{3}=\mathrm{JM}-\mathrm{j} 5+1$

FDI $(J 3, J)=R K D(J 3) *(1,0-T F I(J)) /(A K I(J 3, J, N F)+R K D(J 3))$

TFI(J) = TFI $(J)+F D I(J 3, J)$

IF(IK) $395,3941,3941$

3941 WRITE OUTPUT TAPE $3,530,1(F D I(J, J 3), J 3=1, J, 1, J=2, J M)$

530 FORMAT 1 SHOFDI $=11 \mathrm{~F} 10.71$

395 DO $402 \quad \mathrm{~J} 3=1$.JMS

$J=J M-J 3$

IF $(J-1) \quad 398,398,400$

$398 C I(1)=G F P(N F) * B B(1)$

GO TO 402

$400 \quad C I(J)=\operatorname{GFP}(N F) *(B B(J)-B B(J-1), *(1.0-T F(J))$

402 CONTINUE

DO $404 \mathrm{~J}=3, \mathrm{JM}$

$404 C K(J, J-1)=C I(J-1) *(1.0-(R K D(J-1) /(A K J(J-1, N F)+R K D(J-1))$ DO $406 \quad \mathrm{~J} 3=3 . \mathrm{JM}$ 


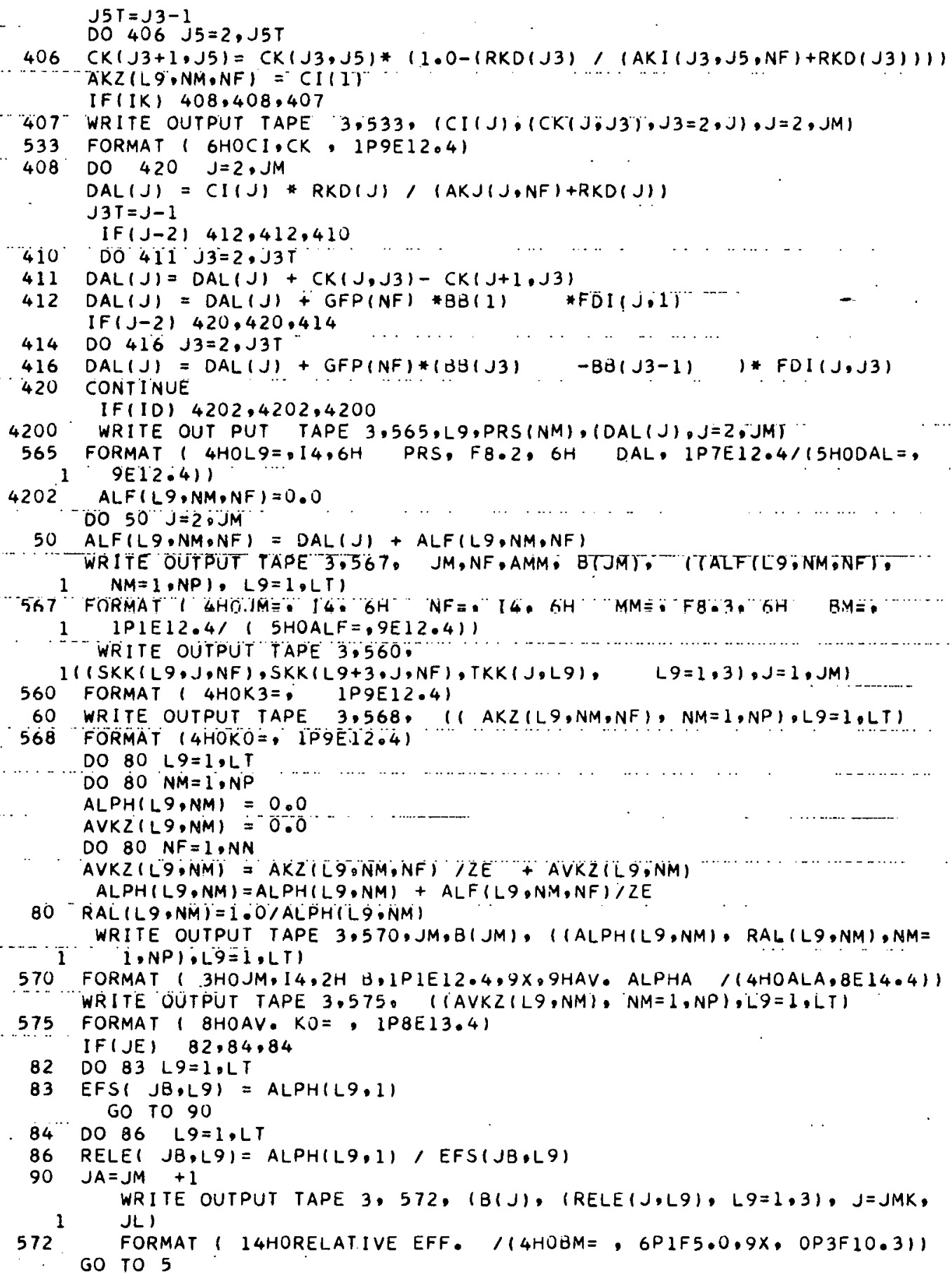


END

SUBROUTINE AVEK2 I NF, JAI

DIMENSION AKJ(35,10), AKI $35,35,10)$, EXM(35), R(35,10), GC(10),

1 ZJE(35), EBB(150), BB(35), AA(10), TC(10), DT(150),GFP(10)

COMMON JM,AKJ,AKI,BB,GC,ZJE,R,AA,TC,GFP, JMS, 10

EXM $(J A-1)=\operatorname{SORTF}(B B(J A-1) * G C(N F)+1.0)$

DO $385 \mathrm{~J}=J A, J M$

$A K J(J, N F)=0.0$

DO $383 \quad J 3=1, J$

$A K I(J, J 3, N F)=0.0$

EXM(J3)= SORTF( BB(.J3) *G(NF)+1.0)

IF $(J)-1) 27.27 \cdot 28$

$27 D B J=8 B(1), Z J E(1)$

GO TO 282

$28 D B J=(B B(J 3) \quad-B B(J 3-1) \quad \mid \quad Z J E(J 3)$

2A? $7 .(M=7, J F(13)+1.2$

$J E M=2 J M$

$E B B(1)=B B(J 3)+0.5 * D B J$

DO $38 \quad \mathrm{~J} 2=2$, JEM

$I E=-2$

$E B B(J 2)=E B B(j 2-1)-O B J$

$E X=S O R T F(E B B(J 2) * G C(N F)+1.0)$

$C H E=11.0+R(J), N F) / A A(N F)) / E X$

$Q 3=C H E * C H E-1.0$

IFIOZ) $30,31,31$

30 WRI TEOUTPUT TAPE 3,540,J,NF,J2,J3,JEM, DB(J),R(J,NF), DOJ, I EBB

1 J $43, J 4=1, j 21$, EXM(J3),EX,CHE, 03

540 FORMAT ( 6HOQ3NEG, 5 I8,1P5E $14.6 /(8 E 14.6)$

$Q 3=0.0$

31 SHE $=S O R T F(03)$

$E C=L O G F(C H E+S H E)$

$T J=T C(N F) *(E X * S H E-E C$.

IF( EX-EXMIJ-I) $33,33,32$

32. TI $=0.0$

60 TO 36

$33^{\circ} \mathrm{CHE}=(1.0+R(J-1, N F) / A A(N F)) / E X$

$Q 2=C H E * C H E-1.0$

IF $O 02134,35,35$

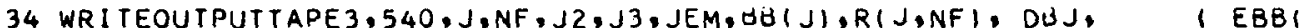

$1 \quad 541, J 4=1, .121$, EXMIJ31,EX, SHF, D?

GO TO 32

35 SHE $=$ SORTF $(02)$

EC=LOGF $(C H E+S H E)$

$T I=T C(N F) *(E X * S H E-E C)$

36

$D T(J 2)=1.0 /(T J-T I)$

IF (IE) $364,364,362$

362 AKJ $(J, N F)=A K J(J, N F)+0.5 * D T(J 2) / 2 J E(J)$

GO

$364 A K I(J, J 3, N F)=A K I(J, J 3, N F)+D T(J 2) / 2 J E(J 3)$

38 CONTINUE

IF (IO) $383,383,381$

381 WRITE OUTPUT TAPE $3,555, \mathrm{~J}, \mathrm{~J} 3,1$ DT(J5),J5=2, JEM)

555 FORMAT, $3 \mathrm{HOJ}=, 14,6 \mathrm{H} J 3=, 14,6 \mathrm{H}$ OT $=, 1 \mathrm{PBE} 12.4 / 13 \mathrm{HODT}$,

$19 E 13.411$ 
$\therefore$

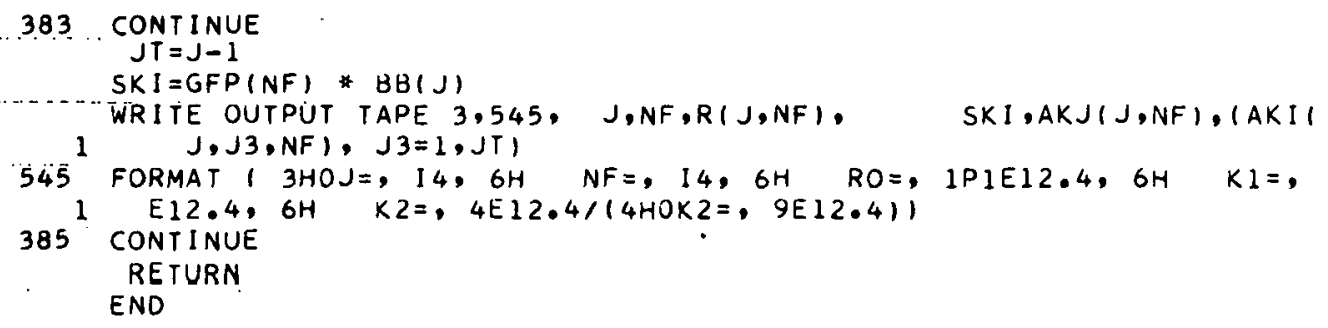




\section{REFERENCES}

1. F. W. Lampe, J. L. Franklin, and F. H. Field, Kinetics of the Reactions of Ions with Molecules, in Progress in Reaction Kinetics, G. Porter, ed. (Pergamon Press, New York, 1961), Vol. 1, p. 68.

2. J. Sayers, Ionic Recombination, in Atomic and Molecular Processes, D. R. Bates, ed. (Academic Press, Inc., New York, 1962), p. 272 .

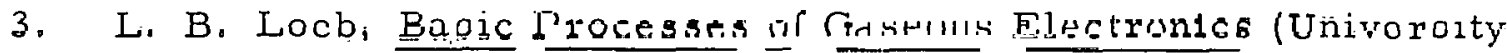
of California Press, Berkeley, 1960), Chap. VI.

4. M. E. Gärdner, Phys. Rev.. 53, 75 (1938).

5. J. Sayers, Proc. Roy. Soc. (London) A169, 83 (1938).

6. T. Y. H. Yeung, Proc. Phys. Soc. (London) 71, 341 (1958).

7. T. Y. H. Yeung, J. Electron. Control 5, 307 (1958).

8. J. J. Thomson, Phil. Mag. 47, 337 (1924).

9. G. L. Natanson, Soviet Phys. - Tech. Phys. 4, 1263 (1960) [Zh. tekh. Fiz. 29, 1373 (1959)].

10. T. Fueno, H. Eyring, and T. Ree, Can. J. Chem. 38, 1693(1960).

11. D. L. Bunker and N. Davidson, J. Am. Chem. Soc. 80, 5090 (1958).

12. K. Watanabe, 'I". Nakayama, and J. Mottl, Final Report on Ionization Potential of Molecules by a Photoionization. Methöd, Department of Physics, University of Hawaii, Dec。1959 (unpublished).

13. M. E. Wacks and M. Krauss, J. Chem. Phys. 35, 1902 (1961).

14. G. G. Cloutier and H. I. Schiff, J. Chem. Phys. 31, 793 (1959).

15. R. C. Gunton and E. C. Y. Inn, J. Chem. Phys. 35, 1896 (1961).

16. L. M. Chanin, A. V. Phelps, and M. A. Biondi, Phys. Rev. 128, 219 (1962).

17. J. P. Doering and B. H. Mahan, J. Chem. Phys. 36, 669 (1962).

18. L. B. Loeb, op. cit. (reference 3), pp. 200-201.

19. P. H. G. Dickinson and J. Sayers, Proc. Phys. Soc. (London) 76, 137 (1960). 
20. R. K. Curran, Phys. Rev. 125, 910 (1962).

21. J. P. Doering, Photochemistry of Some Oxides of Nitrogen (Ph. D. Thesis), University of California, 1961 (unpublished).

22. L. B. Loeb, Fundamental Processes of Electrical Discharge in Gases (John Wiley \& Sons, Inc., New York, 1939), Chap. IX.

23. A. von Engel, in Handbuch der Physik (Springer-Verlag, Berlin, 1956) Vol. 21, p. 504.

24. P. F. Little, in Handbuch der Physik (Springer-Verlag, Berlin, 1956) Vol. 21, p. 57.4.

25. C. W. Park and J. J. Barale, Analog Field Plotter: Description and Use, University of California Radiation Laboratory Report UCRL-8893, March 1960. (unpublished).

26. A. von Engel, Ionized Gases (Oxford University Press, London, 1955), p. 21.

27. J. A. Morrison and D. Edelson, J. Appl. Phys. 33, 1714 (1962).

28. P. Langevin, Ann. chem. et phys. (8) 5, 245 (1905).

29. H. R. Hassé, Phil. Mag. 1, 139 (1926).

30. L. B. Loeb, Basic Processes of Gaseous Electronics (University of California Press, Berkeley, 1960), p. 65.

31. Ibid. p.68.

32. J. O. Hirschfelder, C. F. Curtiss, and R. B. Bird, Molecular Theory of Gases and Liquids (John Wiley \& Sons. Inc. , New York, 1954), pp. 1110-1111.

33. A. M. Tyndall, The Mobility of Positive Ions in Gases (Cambridge University Press, London, 1938), Chap. V; L. B. Loeb; op. cit. (reference 30$),$ p. 56.

34. H. J. Oskam, Philips Res. Repts. 13, 335 (1958).

35. A. M. Tyndall, op. cit. (reference 33), p. 52.

36. E. P. Gray and D. E. Kerr, Ann. Phys. 17, 276 (1962).

37. L. B. Loeb, op. cit. (reference 30), pp. 323-328.

38. Ibid. pp. 545-550:

39. Ibid. pp. 548-549. 
40. Ibid. pp. 541-545.

41. L. D. Landau and E. M. Lifshitz, Mechanics (Addison-Wesley, Reading, Massachusetts, 1960), p.47.

42. L. G: H. Huxley and R. W. Crompton, Elastic Scattering of Electrons, in Atomic and Molecular Processes, D. R. Bates, Ed. (Academic Press, New York, 1962), p. 349.

43. R. D. Present, Kinetic Theory of Gases (McGraw-Hill Book Co., Inc., New York; 1958), p. 142.

44. U. K. Herschbach, 'Lheoretical Analysis of Cross Sections for Chsmiral Rfarction a.s Measured in Mnlerular Pieam Firpeximents, Lawrence Radiation Laboratory Report UCRL-9379, April 1960 (unpublished).

45. L. I. Schiff, Quantum Mechanics (McG̈raw-Hill Book Ċo., Inc., New York, 1955), p.99.

46. L. D. Landau and E. M. Lifshitz, op. cit. (reference 41), p. 36. 47. Ibid. p. 38. 
This report was prepared as an account of Government sponsored work. Neither the United States, nor the Commission, nor any person acting on behalf of the Commission:

A. Makes any warranty or representation, expressed or implied, with respect to the accuracy, completeness, or usefulness of the information contained in this report, or that the use of any information, apparatus, method, or process disclosed in this report may not infringe privately owned rights; or

B. Assumes any liabilities with respect to the use of, or for damages resulting from the use of any information, apparatus, method, or process disclosed in this report.

As used in the above, "person acting on behalf of the Commission" includes any employee or contractor of the Commission, or employee of such contractor, to the extent that such employee or contractor of the Commission, or employee of such contractor prepares, disseminates, or provides access to, any information pursuant to his employment or contract with the Commission, or his employment with such contractor. 